\title{
Left Invariant Evolution Equations on Gabor Transforms.
}

\author{
Remco Duits and Hartmut Fuehr and Bart Janssen \\ Eindhoven University of Technology, Den Dolech 2, 5600 MB Eindhoven, P.O.Box 513 The Netherlands. \\ Department of Mathematics and Computer Science, CASA applied analysis, \\ Department of Biomedical Engineering, BMIA Biomedical image analysis. \\ e-mail: R.Duits@tue.nl, B.J.Janssen@tue.nl \\ Lehrstuhl A für Mathematik, RWTH Aachen University, 52056 Aachen, Germany. \\ e-mail: fuehr@MathA.rwth-aachen.de
}

23rd February 2009

\begin{abstract}
By means of the unitary Gabor transform one can relate operators on signals to operators on the space of Gabor transforms. In order to obtain a translation and modulation invariant operator on the space of signals, the corresponding operator on the reproducing kernel space of Gabor transforms must be left invariant, i.e. it should commute with the left regular action of the Heisenberg group. By using the left invariant vector fields on $H_{3}$ and the corresponding left-invariant vector fields on a cross-section of the phase space $H_{3} / \Theta$ in the generators of our transport and diffusion equations on Gabor transforms we naturally employ the essential group structure on the domain of a Gabor transform. We shall use these evolutions for three different tasks. First, there is the task of enhancing Gabor transforms (and corresponding signals) by means of non-linear left invariant diffusion. Secondly, there is the task of non-linear adaptive left-invariant convection (reassignment) towards the most probable curves, while maintaining the original signal. Finally, there is the task of extracting the most probable curves in the Gabor domain.
\end{abstract}

Keywords: The Heisenberg group $H_{3}$, Left invariant evolution equations on $H_{3}$, Re-assignment in Gabor Analysis, Evolutions on phase space, Contact-differential geometry on $\mathrm{H}_{3}$.

\section{Introduction}

To get an overview of how a signal is composed out of local frequencies one usually constructs a Gabor transform of the signal. As the Gabor transform is a unitary operator (preserving the $\mathbb{L}_{2}$-norm) one can robustly relate operators on Gabor transforms to operators on signals. In this article we consider suitable adaptive convection-diffusion operators on Gabor transforms, while keeping track of the corresponding operator on the signal.

The Gabor transform was first proposed in 1964 in a discrete setting [21, 43]. Later it was formulated in a continuous setting [26] showing its close resemblance to the short-time Fourier transform. It was already in the mid 1970's that Kodera et al. [33] noticed the need for a further enhancement of the spectrogram that is obtained from the short-time Fourier transform of a signal by taking its squared modulus. They noticed that, due to the uncertainty principle, there is always a tradeoff between say time and frequency resolution in the short-time Fourier transform. As a result the spectrogram shows a "blurred" version of the true spectral density. The modified moving-window method they propose as a solution to this problem indeed sharpens the 
spectrogram and thereby increases its readability. However, their modification only handles the square modulus of the short-time Fourier transform of a signal and loses the phase information. Therefore it is not possible to reconstruct the signal from its modified moving-window method representation. The improvements of the method by Auger and Flandrin [2] yielded computational advantages in the Gabor domain but still their operators in the Gabor transform do not yield natural effective operators in the actual signal domain.

A method called differential reassignment was later developed by Chassande-Mottin et al. [7]. This method, next to its invertibility, also produces a vector field along which the spectrogram is continuously deformed. Hence the term differential reassignment for this adaptive convection on Gabor transforms. Later on Daudet et al.[9] reported the relevance of the Heisenberg group structure in the domain of a Gabor transform, but they proposed differential re-assignment in a phase-invariant way and consequently (as we will show both theoretically as practically in this article) their method exactly coincides with the approach Chassande-Mottin et al. [7]. Furthermore, we will show that for Gabor-transforms with Gaussian windows it relates to a simple erosion operator (well-known in image processing [5]) on the modulus followed by a restoration of phase afterwards.

In this article we initially follow the group theoretical approach by Daudet et al.[9] and operationalize their generators in phase space. However, in contrast to the work of Daudet et al., we will make a clear distinction between phase-covariant and phase-covariant convection and diffusion operators on Gabor transforms. The latter approach allows us to keep track of the corresponding operators in the signal domain. Here we follow the same group theoretical approach as in our recent works of line/contour enhancement and completion via invertible orientation scores, $[13,19,11,12,15,16]$, (where we had to deal with the Euclidean motion group rather than the Heisenberg group). This shows the wide applicability of our general approach, [14], on left-invariant convection-diffusions on Lie groups in signal and image processing.

In the continuous setting the Gabor-transform $\mathcal{G}_{\psi}(f): \mathbb{R}^{d} \times \mathbb{R}^{d} \rightarrow \mathbb{C}$ of a square integrable signal $f: \mathbb{R}^{d} \rightarrow \mathbb{C}$ (we mainly consider the case $d=1$ ) is commonly defined as

$$
\mathcal{G}_{\psi}^{n}(f)(p, q)=\int_{\mathbb{R}^{d}} f(\xi) \overline{\psi(\xi-p)} e^{-2 \pi n i(\xi-p) \cdot q} \mathrm{~d} \xi, n \in \mathbb{Z},
$$

but is also often defined as

$$
\mathbb{G}_{\psi}^{n}(f)(p, q)=\int_{\mathbb{R}^{d}} f(\xi) \overline{\psi(\xi-p)} e^{-2 \pi n i \xi \cdot q} \mathrm{~d} \xi, n \in \mathbb{Z}
$$

and (surprisingly enough) only rarely defined as $\mathcal{W}_{\psi}^{n} f: \mathbb{R} \times \mathbb{R} \times(\mathbb{R} / \mathbb{N}) \rightarrow \mathbb{C}$ given by

$$
\mathcal{W}_{\psi}^{n} f(p, q, s)=e^{-2 \pi i n\left(s+\frac{p q}{2}\right)} \int_{\mathbb{R}} f(\xi) \overline{\psi(\xi-p)} e^{-2 \pi i(\xi-p) q n} \mathrm{~d} \xi, \quad p, q \in \mathbb{R}^{d}, s \in \mathbb{R} / \mathbb{N},
$$

again indexed by $n \in \mathbb{Z}$. Note that

$$
\mathcal{G}_{\psi}^{n}(f)(p, q)=\mathcal{W}_{\psi}^{\lambda} f\left(p, q, s=-\frac{p q}{2}\right) \text { and } \mathbb{G}_{\psi}^{n}(f)(p, q)=\mathcal{W}_{\psi}^{\lambda} f\left(p, q, s=\frac{p q}{2}\right) .
$$

Typically the choice of convention does not matter if one only performs signal processing on the modulus and if one simply ignores the phase. However, the phase information is often rather important, as can be easily seen from a simple experiment. Typically, if one considers the discrete Fourier transform say of a finite $2 D$-signal (image), takes the phase (setting the modulus to 1 ) and applies an inverse discrete Fourier transform the output still reflects the essential structures in the signal. However, the output of the net operator that arises by just taking the modulus (setting the phase to zero) in the discrete Fourier domain usually usually affects a signal dramatically, see Fig. 1.

To "cope" with this problem various literature has appeared on phase invariant re-assignment operators in the Gabor domain. Here one restores the old phase of a Gabor transform, while applying convection to the modulus which is considered as a function on the additive group $\mathbb{R}^{2 d}$. 

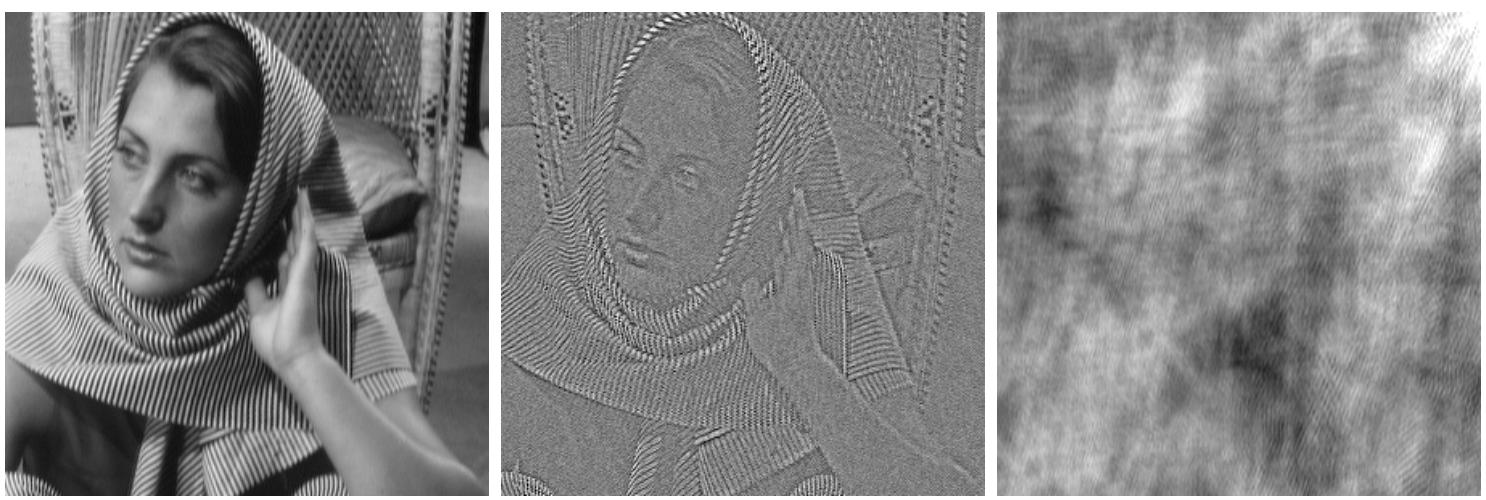

Figure 1: Simple experiment to illustrate the relevance of phase. Left: $200 \times 200$ fragment of the Barbara $512 \times 512$-image $\mathbf{f}$. Removing the amplitude in the Discrete Fourier Transform (i.e. $D F T^{-1}\left[|D F T \mathbf{f}|^{-1} D F T \mathbf{f}\right] \quad$ ) still reveals the essential structure of the image-fragement (middle image), whereas removing the phase (i.e. $D F T^{-1}[|D F T \mathbf{f}|]$ ) entirely destroys the image (right image).

Equivalently, one may consider phase invariant operators in the Gabor domain. However, the big drawback of such an approach is that one does not preserve the meaning of the Gabor-atoms in the wavelet domain, so for example edges interfere with lines and it is not possible to restore the output if using a different reference to shape. This is clearly illustrated in Figure 2. Furthermore, the underlying non-commutative group-structure of spatial shifts and frequency shifts is entirely destroyed in such approach. Recall to this end the well-known uncertainty principle in the signal domain

$$
\operatorname{Var}(\xi) \operatorname{Var}\left(-i \partial_{\xi}\right):=\int_{\mathbb{R}} \xi^{2}|\psi(\xi)|^{2} \mathrm{~d} \xi \int_{\mathbb{R}} \omega^{2}|\mathcal{F} \psi(\omega)|^{2} \mathrm{~d} \omega \geq \frac{1}{4}\left(\int_{\mathbb{R}}|\psi(\xi)|^{2} \mathrm{~d} \xi\right)^{2}=:\left(\frac{1}{2 i} \mathrm{E}\left(\left[\xi,-i \partial_{\xi}\right]\right)\right)^{2}
$$

where $\mathcal{F} \psi$ is the Fourier transform of $\psi$ given by $\mathcal{F} \psi(\omega)=\frac{1}{\sqrt{2 \pi}} \int_{\mathbb{R}} \psi(\xi) e^{-i \omega \xi} \mathrm{d} \xi$. Here we note that the commutator of the translation generator and the modulation generator $\left[\xi,-i \partial_{\xi}\right]=\xi \circ\left(-i \partial_{\xi}\right)-\left(-i \partial_{\xi}\right) \circ \xi$ equals $i$ so the uncertainty between time and frequency is a direct consequence of the noncommutative nature of the $2 d+1$-dimensional reduced Heisenberg group $H_{2 d+1}, d=1$, and corresponding reduced Weyl-Heisenberg group $H_{r}=H_{2 d+1} /(\{0\} \times\{0\} \times \mathbb{Z})$. Such uncertainty relation also apply to the Gabor domain. This is similar to using the infinitesimal generators of actions (modulations and translations) of the Heisenberg group on the space of signals $\mathbb{L}_{2}\left(\mathbb{R}^{d}\right)$. In general for all pairs of self-adjoint operators $A, B$ on a Hilbert space $H$ one has

$$
(A U, A U)_{H}(B U, B U)_{H} \geq\left(\frac{1}{2 i}(\psi,[A, B] \psi)_{H}\right)^{2},
$$

so we may as well set $H=\mathbb{L}_{2}\left(H_{r}\right)$ and $A=i \mathrm{~d} \mathcal{R}\left(A_{i}\right)$ and $B=i \mathrm{~d} \mathcal{R}\left(A_{i+d}\right)$, where $\mathrm{d} \mathcal{R}$ denotes the derivative of the right-regular representation $\mathcal{R}: H_{r} \rightarrow \mathcal{B}\left(\mathbb{L}_{2}\left(H_{r}\right)\right)$ which is given by

$$
\begin{aligned}
& \mathcal{R}_{g} U(h)=U(h g), \text { for all } h, g \in H_{2 d+1} \text { and all } U \in \mathbb{L}_{2}\left(H_{2 d+1}\right) \\
& \mathrm{d} R\left(A_{i}\right) U(g)=\lim _{\epsilon \rightarrow 0} \frac{\phi\left(g e^{t A_{i}}\right)-U(g)}{\epsilon} \text { for all } g \in H_{2 d+1} \text { and smooth } U \in \mathcal{C}^{\infty}\left(H_{r}\right),
\end{aligned}
$$

of $H_{r}$ on $\mathbb{L}_{2}\left(H_{r}\right)$, where $\mathcal{B}\left(\mathbb{L}_{2}\left(H_{r}\right)\right)$ denotes the space of bounded, linear operators on $\mathbb{L}_{2}\left(H_{r}\right)$ and where $A_{i}=\partial_{p_{i}}, A_{i+d}=\partial_{q_{i}}$ are elements in the Lie-algebra $T_{e}\left(H_{r}\right)$ and obtain the uncertainty principle in the Gabor domain:

$\mathrm{E}\left(\left(\mathrm{d} \mathcal{R}\left(A_{1}\right)\right)^{2}\right) \mathrm{E}\left(\left(\mathrm{d} \mathcal{R}\left(A_{2}\right)\right)^{2}\right) \geq\left(\frac{1}{2 i} \mathrm{E}\left(\left[\mathrm{d} \mathcal{R}\left(A_{1}\right), \mathrm{d} \mathcal{R}\left(A_{2}\right)\right]\right)\right)^{2}=\left(\frac{1}{2 i} \mathrm{E}\left(\left[\mathrm{d} \mathcal{R}\left(\left[A_{1}, A_{2}\right]\right)\right]\right)^{2}=\left(\frac{1}{2 i} \mathrm{E}\left(\mathrm{d} \mathcal{R}\left(A_{3}\right)\right)^{2}\right.\right.$

where again $E$ denotes expected value and where the probability density in the Gabor domain is given by

$$
(p, q, s) \mapsto \frac{1}{\left(\mathcal{W}_{\psi} f, \mathcal{W}_{\psi} f\right)}\left|\mathcal{W}_{\psi} f(p, q, s)\right|^{2},
$$



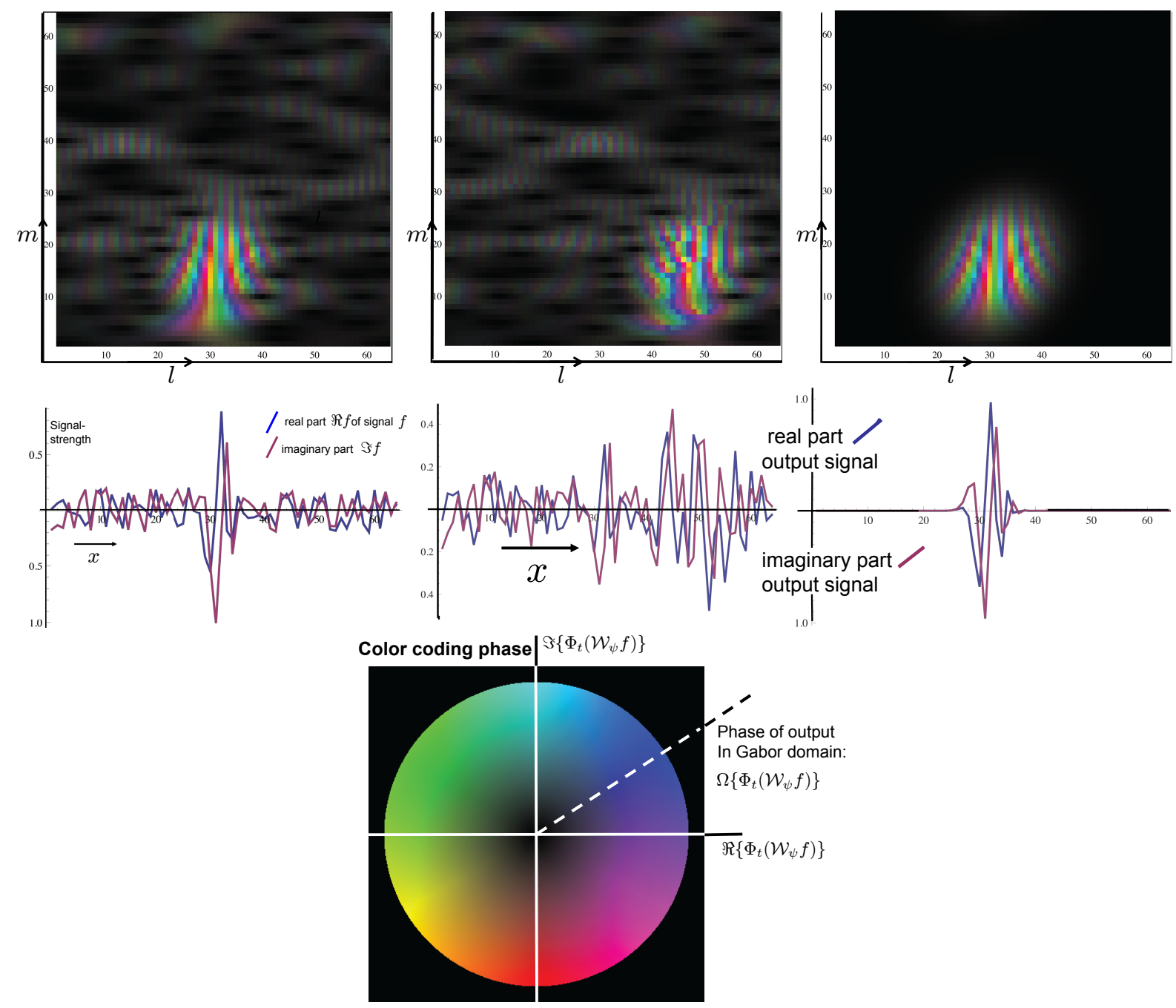

Figure 2: Top row from left to right, (1) the Gabor transform of original signal $f,(2)$ processed Gabor transform $\Phi_{t}\left(\mathcal{W}_{\psi} f\right)$ where $\Phi_{t}$ denotes a phase invariant shift (for more elaborate adaptive convection/re-assignment operators see Section 5 where we operationalize the theory in [9]) using a discrete Heisenberg group, where $l$ represents discrete spatial shift and $m$ denotes discrete local frequency, (3) processed Gabor transform $\Phi_{t}\left(\mathcal{W}_{\psi} f\right)$ where $\Phi_{t}$ denotes a phase covariant diffusion operator on Gabor transforms with stopping time $t>0$ as explained in Section 7 . Note that phase-covariance is preferable over phase invariance. For example restoration of the old phase in the phase invariant shift (the same holds for the adaptive phase-invariant convection of Section 5 ) creates noisy artificial patterns (middle image) in the phase of the transported strong responses in the Gabor domain. Middle row, from left to right : (1) Original complex-valued signal $f$, (2) output signal $\Upsilon_{\psi} f=\mathcal{W}_{\psi}^{*} \Phi_{t} \mathcal{W}_{\psi} f$ where $\Phi_{t}$ denotes a phase-invariant spatial shift (due to phase invariance the output signal looks bad and clearly phase invariant spatial shifts in the Gabor domain do not correspond to spatial shifts in the signal domain), (3) Output signal $\Upsilon_{\psi} f=\mathcal{W}_{\psi}^{*} \Phi_{t} \mathcal{W}_{\psi} f$ where $\Phi_{t}$ denotes phase-covariant adaptive diffusion in the Gabor domain with stopping time $t>0$. Bottom row: explanation color-coding of the phase in the corresponding Gabor transforms in the top row. 
just like $\xi \mapsto \frac{1}{(f, f)}|f(\xi)|^{2}$ represents the probability density in the signal domain.

As we will see in section 2 the Gabor transform as defined in (1.1) may be written as

$$
\mathcal{W}_{\psi}^{n} f(p, q, s)=\left(\mathcal{U}_{(p, q, s)}^{n} \psi, f\right)_{\mathbb{L}_{2}\left(\mathbb{R}^{2}\right)},
$$

where $\mathcal{U}: H_{r} \rightarrow B\left(\mathbb{L}_{2}\left(\mathbb{R}^{d}\right)\right)$ denotes the well-known Schrodinger representation of the WeylHeisenberg group (or reduced Heisenberg group $H_{r}=H_{2 d+1} /\{0\} \times\{0\} \times \mathbb{Z}$ ). This puts an important group-structure on the domain of a Gabor transform. The representation $\mathcal{U}^{n}, n \in \mathbb{Z}$ is square integrable representation with respect to the quotient $H_{r} / \Theta$ (where $\Theta$ denotes the phase subgroup $\left.\left.\left\{\left(0,0, e^{2 \pi i s}\right) \mid s \in[0,1)\right\}\right)\right)$ with invariant measure $\mathrm{d} \mu_{H_{r} / \Theta}(g)=\mathrm{d} q \mathrm{~d} p$. Therefore by the theory of coherent states, [47], we employ that

$$
\int_{\mathbb{R}^{d}} \int_{\mathbb{R}^{d}}\left|\mathcal{W}_{\psi}[f](p, q, s)\right|^{2} \mathrm{~d} p \mathrm{~d} q=C_{\psi} \int_{\mathbb{R}^{d}}|f(p)|^{2} \mathrm{~d} p,
$$

for all $f \in \mathbb{L}_{2}\left(\mathbb{R}^{d}\right), s>0$ and for all $\psi \in \mathbb{L}_{2}\left(\mathbb{R}^{d}\right)$, where the constant $C_{\psi}$ is given by

$$
C_{\psi}=\int_{\mathbb{R}^{d}} \int_{\mathbb{R}^{d}}\left|\left(\mathcal{U}_{(p, q, 0)}^{n} \psi, \psi\right)\right|^{2} \mathrm{~d} p \mathrm{~d} q=\frac{1}{n}\|\psi\|_{\mathbb{L}_{2}\left(\mathbb{R}^{d}\right)}^{2}<\infty \quad n \in \mathbb{Z},
$$

for all $\psi \in \mathbb{L}_{2}\left(\mathbb{R}^{2}\right)$. Consequently, we may reconstruct via

$$
f(\xi)=\frac{1}{C_{\psi}} \int_{0}^{1} \int_{\mathbb{R}^{d}} \int_{\mathbb{R}^{d}}\left(\mathcal{W}_{\psi} f\right)(p, q, s) e^{i 2 \pi n[(\xi, q)+(s)-(1 / 2)(p, q)]} \psi(\xi-p) \mathrm{d} p \mathrm{~d} q \mathrm{~d} s .
$$

Now $H_{2 d+1} / \Theta \equiv \mathbb{R}^{2}$ and in principle (if no operators are applied to the Gabor transform) the integration over $s$ in (1.6) is redundant as the integrand is independent on $s>0$.

However as soon as we relate operators $\Phi: \mathcal{R}\left(\mathcal{W}_{\psi}^{n}\right) \rightarrow \mathbb{L}_{2}\left(H_{r}\right)$ on Gabor transforms, which actually use and change the relevant phase information of a Gabor transform, in a well-posed manner to operators $\Upsilon_{\psi}: \mathbb{L}_{2}\left(\mathbb{R}^{d}\right) \rightarrow \mathbb{L}_{2}\left(\mathbb{R}^{d}\right)$ on signals via

$$
\begin{aligned}
& \left(\Upsilon_{\psi} f\right)(\xi)=\left(\mathcal{W}_{\psi}^{*} \circ \Phi \circ \mathcal{W}_{\psi} f\right)(\xi) \\
& =\frac{1}{C_{\psi}} \int_{0}^{1} \int_{\mathbb{R}^{d}} \int_{\mathbb{R}^{d}}\left(\Phi\left(\mathcal{W}_{\psi} f\right)\right)(p, q, s) e^{i 2 \pi n[(\xi, q)+(s)-(1 / 2)(p, q)]} \psi(\xi-p) \mathrm{d} p \mathrm{~d} q \mathrm{~d} s,
\end{aligned}
$$

the integration over $s>0$ does make a difference. In such cases, as we shall consider in section 6 and section 7 the $s$-variable keeps track of the phase and should be considered as a buffer to store the non-commuting behavior of both position- and frequency shifts.

In this article we shall consider operator's $\Phi$ as left-invariant evolution operators with stopping time $t>0$. To stress the dependence on the stopping time we shall write $\Phi_{t}$ rather than $\Phi$. Typically, such operators are defined by $W(p, q, s, t)=\Phi_{t}\left(\mathcal{W}_{\psi} f\right)(p, q, s)$ where $W$ is the solution of

$$
\begin{cases}\partial_{t} W(p, q, s, t) & =Q\left(\left|\mathcal{W}_{\psi} f\right|, \mathcal{A}_{1}, \ldots, \mathcal{A}_{d}, \mathcal{A}_{d+1}, \ldots, \mathcal{A}_{2 d}\right) W(p, q, s, t) \\ W(p, q, s, 0) & =\mathcal{W}_{\psi} f(p, q, s)\end{cases}
$$

where we note that the left-invariant vector fields $\left\{\mathcal{A}_{i}\right\}_{i=1}^{2 d+1}$ on $H_{r}$ are given by

$$
\mathcal{A}_{i}=\partial_{p_{i}}+\frac{q_{i}}{2} \partial_{s}, \mathcal{A}_{d+i}=\partial_{q_{i}}-\frac{p_{i}}{2} \partial_{s}, \mathcal{A}_{2 d+1}=\partial_{s}, \quad \text { for } i=1, \ldots, d,,
$$

with left-invariant differential form

$$
Q\left(\left|\mathcal{W}_{\psi} f\right|, \mathcal{A}_{1}, \ldots, \mathcal{A}_{d}, \mathcal{A}_{d+1}, \ldots, \mathcal{A}_{2 d}\right)=-\sum_{i=1}^{2 d} a_{i}\left(\left|\mathcal{W}_{\psi} f\right|\right)(p, q) \mathcal{A}_{i}+\mathcal{A}_{i} D_{i j}\left(\left|\mathcal{W}_{\psi} f\right|\right)(p, q) \mathcal{A}_{j}
$$

and $a_{i}$ and $D_{i j}$ functions such that $(p, q) \mapsto a_{i}\left(\left|\mathcal{W}_{\psi} f\right|\right)(p, q) \in \mathbb{R}$ and $(p, q) \mapsto a_{i}\left(\left|\mathcal{W}_{\psi} f\right|\right)(p, q) \in \mathbb{R}$ are differentiable and $D^{T}=D>0$ (with $D=\left[D_{i j}\right]$ ) for all $i=1, \ldots, 2 d, j=1, \ldots 2 d$. 
Next we provide a brief motivation for considering these operators $\Phi_{t}$. First of all the operator $\Phi_{t}$ should be left-invariant, i.e.

$$
\mathcal{L}_{g} \circ \Phi_{t}=\Phi_{t} \circ \mathcal{L}_{g} \quad \text { for all } g \in H_{r} \text { all } t>0,
$$

where the left-regular representation is given by

$$
\mathcal{L}_{g} U(h)=U\left(g^{-1} h\right), \text { for all } h, g \in H_{r} \text { and all } U \in \mathbb{L}_{2}\left(H_{r}\right),
$$

in order to ensure, [14], that the net operator $\Upsilon_{\psi}$ given by (1.7) satisfies

$$
\Upsilon_{\psi} \circ \mathcal{U}_{g}=\mathcal{U}_{g} \circ \Upsilon_{\psi} \text { for all } g \in H_{r}
$$

Secondly, we note that some of the functions $a_{i}, D_{i j}$ may be constant (in particular zero), but not of all of them. If they are all constant then it directly follows by linearity, (1.10) and the extended Schur's lemma [10] that $\Upsilon_{\psi}$ is a constant times the identity. So that we need adaptive convection and/or adaptive diffusion via an adpative conductivity matrix $D$ similar to our previous work framework on adaptive diffusions invertible orientation scores [18], [11], [16], [12] (where we replaced $H_{r}$ by the 2D-Euclidean motion group).

Thirdly, we consistently removed the $\mathcal{A}_{2 d+1}$-direction in diffusion and convection and removed the $s$-dependence in the coefficients $a_{i}\left(\left|\mathcal{W}_{\psi} f\right|\right)(p, q), D_{i j}\left(\left|\mathcal{W}_{\psi} f\right|\right)(p, q)$ of the generator $Q\left(\left|\mathcal{W}_{\psi} f\right|, \mathcal{A}_{1}, \ldots, \mathcal{A}_{d}, \mathcal{A}_{d+1}, \ldots, \mathcal{A}_{2 d}\right)$ by taking the absolute value $\left|\mathcal{W}_{\psi} f\right|$ which, in contrast to $\mathcal{W}_{\psi} f$, does not depend on $s>0$. Here we take the absolute value to adaptively steer the diffusion and convection to avoid oscillations. ${ }^{1}$ Furthermore we would like to enforce horizontal diffusion and convection, which means that transport and diffusion takes place along horizontal curves in $H_{r}$ which are curves $t \mapsto(p(t), q(t), s(t)) \in H_{r}$ along which

$$
s(t)=\frac{1}{2} \int_{0}^{t} \sum_{i=1}^{d} p_{i}(\tau) q_{i}^{\prime}(\tau)-p^{\prime}(\tau) q_{i}(\tau) \mathrm{d} \tau .
$$

This gives a nice geometric interpretation to the phase variable $s(t)$, since by the Stokes theorem it represents the net surface area between a straight-line connection between $(p(0), q(0), s(0))$ and $(p(t), q(t), s(t))$ and the actual horizontal curve connection $[0, t] \ni \tau \mapsto(p(\tau), q(\tau), s(\tau))$. For details, see Appendix B. So by omitting the $\mathcal{A}_{2 d+1}=\partial_{s}$ direction in the generator of the non-linear left-invariant convection and diffusions, like in (1.8), the $s$-axis keeps track of the non-commutative nature between both spatial- and frequency-shifts. Here we stress that the omission of $\mathcal{A}_{2 d+1}$ does not affect the smoothness and uniqueness of the solutions of (1.8), since the initial condition is for suitable choice of $\psi$ (for example a Gaussian) infinitely differentiable and the Hörmander condition [28], [14] is still satisfied, since clearly $\left[\mathcal{A}_{i 1}, \mathcal{A}_{i+d}\right]=-\mathcal{A}_{2 d+1}, i=1, \ldots, d$.

The domain of a (processed) Gabor transform $\Phi_{t}\left(\mathcal{W}_{\psi} f\right)$ should not be considered as $\mathbb{R}^{2} \equiv$ $H_{r} / \Theta$. It is not even entirely appropriate to consider it as the reduced Heisenberg group $H_{r}=$ $H_{2 d+1} /\{0\} \times\{0\} \times \mathbb{Z}$, because of the horizontality constraint. As we explain in Appendix B and Appendix $\mathrm{C}$ it should be considered as a principal fiber bundle $P_{T}=\left(H_{r}, \mathbb{T}, \pi, \mathcal{R}\right)$ equipped with the Cartan connection form $\omega_{g}\left(X_{g}\right)=\left\langle\mathrm{d} s+\frac{1}{2}(p \mathrm{~d} q-q \mathrm{~d} p), X_{g}\right\rangle$ or equivalently as a contact manifold $\left(H_{r}, \mathrm{~d} \mathcal{A}^{3}=\mathrm{d} s+\frac{1}{2}(q \mathrm{~d} p-p \mathrm{~d} q)\right)$ of dimension 3 .

Throughout this article we shall use this underlying differential geometry for underpinning of our specific choices of the adaptive coefficients $a_{i}$ and $D_{i j}$. Here we shall distinguish between two approaches. Either we choose $a_{i}$ and $D_{i i}$ such that the phase is preserved, allowing us to restrict ourselves to phase space, leading to explicit phase invariant schemes of for example the convection PDE's proposed in [9]. Or we choose $a_{i}$ and $D_{i j}$ such that the phase is transported along the characteristic curves of convection and diffusion, which we shall call phase covariant schemes.

\footnotetext{
${ }^{1}$ This was also essential in the context of non-linear left-invariant diffusions on invertible orientation scores, [12], [18].
} 


\section{The Gabor transform and phase space}

Let $H_{3}$ be the Heisenberg group consisting of elements $(p, q, s) \in \mathbb{R}^{3}$ and group product

$$
(p, q, s)\left(p^{\prime}, q^{\prime}, s^{\prime}\right)=\left(p+p^{\prime}, q+q^{\prime}, s+s^{\prime}+\frac{1}{2} \Im\left\{(p+i q)\left(p^{\prime}+i q^{\prime}\right)\right\}\right)=\left(p+p^{\prime}, q+q^{\prime}, s+s^{\prime}+\frac{1}{2}\left(q p^{\prime}-p q^{\prime}\right)\right) .
$$

Let $\left\{\partial_{p}, \partial_{q}, \partial_{s}\right\}$ be a basis of the Lie-algebra $T_{e}\left(H_{3}\right)$ attached to the unity element $e=(0,0,0)$. So for example $\partial_{p}$ denotes the tangent vector at $e$ along the curve $\{(p, 0,0) \mid p \in \mathbb{R}\}$. Here we note that tangent vectors can always be identified with differential operators on locally defined smooth functions. The vector space $T_{e}\left(H_{3}\right)$ is a Lie-algebra under the Lie product (for explanation on this formula, see Appendix G)

$$
[A, B]=\lim _{t \rightarrow 0} t^{-2}\left(a(t) b(t)(a(t))^{-1}(b(t))^{-1}-e\right),
$$

where $a$ and $b$ are arbitrary curves in $H_{3}$ such that their tangent vector at the unity element equals respectively $A$ and $B$. From now on we shall write

$$
A_{1}=\partial_{p}, A_{2}=\partial_{q}, A_{3}=\partial_{s} .
$$

It is not difficult to verify that

$$
\left[A_{1}, A_{3}\right]=\left[A_{2}, A_{3}\right]=0 \text { and }\left[A_{1}, A_{2}\right]=-A_{3}
$$

The exponential map from $T_{e}\left(H_{3}\right)$ onto $H_{3}$ is surjective and it is simply given by

$$
\exp \left(t \sum_{i=1}^{3} a_{i} A_{i}\right)=\left(a_{1} t, a_{2} t, a_{3} t\right) \in H_{3} .
$$

Here we note that in literature it is also common to work with a non-symmetric parametrization of the Heisenberg group. For example [9], where one must set $\phi=s+\frac{p q}{2}, b=p, v=q$. This different parametrization comes from two fundamental ways to parameterize nilpotent group elements, namely by coordinates of the first and second kind:

$$
g=\exp \left(\sum_{i=1}^{3} a_{i} A_{i}\right)=\prod_{i=1}^{3} \exp \left(b_{i} A_{i}\right),
$$

where by the CBH-formula one must set $a_{1}=p, a_{2}=q, a_{3}=s$ and

$$
b_{1}=p, b_{2}=q \text { and } b_{3}=s+\frac{p q}{2} .
$$

In the sequel it will become apparent why we use the coordinates of the first kind. The main reason is that the exponential map becomes the identity, so that exponential curves (along which the left-invariant convection/diffusion will take place) are straight-lines in $\mathbb{R}^{3}$ if we embed $H_{3}$ in $\mathbb{R}^{3}$.

Now consider the following unitary, irreducible, representations $\mathcal{U}^{\lambda}: H_{3} \rightarrow \mathcal{B}\left(\mathbb{L}_{2}\left(\mathbb{R}^{2}\right)\right)$,

$$
\mathcal{U}_{g=(p, q, s)}^{\lambda} \psi(\xi)=e^{2 \pi i \lambda\left(s+q \xi-\frac{p q}{2}\right)} \psi(\xi-p), \quad \psi \in \mathbb{L}_{2}(\mathbb{R}),
$$

of the Heisenberg group $H_{3}$, parameterized by $\lambda \in \mathbb{R}$. So these representations are a composition of a modulation and a translation of a signal.

The derivative of these representations puts an isomorphism between the Lie-algebra $T_{e}\left(H_{3}\right)=$ $\operatorname{span}\left\{A_{1}, A_{2}, A_{3}\right\}$ and the Lie-algebra $\operatorname{span}\left\{\mathrm{d} \mathcal{U}\left(A_{1}\right), \mathrm{d} \mathcal{U}\left(A_{2}\right), \mathrm{d} \mathcal{U}\left(A_{3}\right)\right\}$ (equipped with the usual Lie-bracket $[A, B]=A B-B A)$ of the following differential operators on smooth signals:

$$
\begin{aligned}
& \mathrm{d} \mathcal{U}\left(A_{1}\right) \phi(x)=\left(\lim _{\epsilon \rightarrow 0} \frac{\mathcal{U}_{\exp \left(\epsilon A_{1}\right)}^{\lambda} \phi-\phi}{\epsilon}\right)(x)=\lim _{\epsilon \rightarrow 0} \frac{\phi(x-\epsilon)-\phi(x)}{\epsilon}=-\partial_{x} \phi(x) \\
& \mathrm{d} \mathcal{U}\left(A_{2}\right) \phi(x)=\left(\lim _{\epsilon \rightarrow 0} \frac{\mathcal{U}_{\exp \left(\epsilon A_{2}\right)}^{\lambda} \phi-\phi}{\epsilon}\right)(x)=\lim _{\epsilon \rightarrow 0} \frac{e^{2 \pi i \lambda \epsilon x} \phi(x)-\phi(x)}{\epsilon}=2 \pi i \lambda x \phi(x) \\
& \mathrm{d} \mathcal{U}\left(A_{3}\right) \phi(x)=2 \pi i \lambda \phi(x)
\end{aligned}
$$


for all $\phi \in \mathbb{L}_{2}(\mathbb{R}) \cap \mathcal{S}(\mathbb{R})$. It is well-known that the uncertainty between frequency (impuls) and position is due to the non commutative nature of $H_{3}$, recall that

$$
\begin{aligned}
& \operatorname{Var}(P) \operatorname{Var}(Q):=\int_{\mathbb{R}} t^{2}|\psi(t)|^{2} \mathrm{~d} t \int_{\mathbb{R}} \omega^{2}|\mathcal{F} \psi|^{2}(\omega) \mathrm{d} \omega \geq \\
& \frac{1}{4}\left(\int_{\mathbb{R}}|\psi(t)|^{2} \mathrm{~d} t\right)^{2}=:\left(\frac{1}{2 i} \mathrm{E}\left(\left[x,-i \partial_{x}\right]\right)\right)^{2}=\left(\frac{1}{4 \pi \lambda i} \mathrm{E}\left(\left[\mathrm{d} \mathcal{U}^{\lambda}\left(A_{1}\right), \mathrm{d} \mathcal{U}^{\lambda}\left(A_{2}\right)\right]\right)\right)^{2} \\
& =\left(\frac{1}{4 \pi \lambda i} \mathrm{E}\left(\left[\mathrm{d} \mathcal{U}^{\lambda}\left(\left[A_{1}, A_{2}\right]\right)\right]\right)^{2}=\left(\frac{1}{4 \pi \lambda i} \mathrm{E}\left(\mathrm{d} \mathcal{U}^{\lambda}\left(A_{3}\right)\right)^{2} .\right.\right.
\end{aligned}
$$

The big problem however, is that these differential operators $\left\{\mathrm{d} \mathcal{U}\left(A_{i}\right)\right\}_{i=1}^{3}$ are not well-defined at locations in a signal $x \mapsto f(x)$ where multiple patches $\mathcal{U}_{p, q, s} \psi$ may be present at a single location $p \in \mathbb{R}$ causing inteference in the out-put of the infinitesimal generators of translation and modulation. To cope with this practical problem one should consider a Gabor domain transform $\mathcal{W}_{\psi} f$ of a signal, constructed from a low-pass filter (usually a Gaussian kernel as this minimizes the uncertainty, in the sense that (2.17) holds with equality), to obtain a "score" of local positions and frequencies. This is done by $\mathcal{W}_{\psi}^{\lambda} f(p, q, s)=\left(\mathcal{U}_{g}^{\lambda} \psi, f\right)_{\mathbb{L}_{2}(\mathbb{R})}$, with $g=(p, q, s) \in H_{3}$, i.e. by (1.1).

Now typically (like in the framework of invertible orientation scores, cf. [12, 19]) in this score local patches are manifestly torn apart and therefore the left-invariant generators on Gabor transforms $\left\{\mathrm{d} \mathcal{R}\left(A_{i}\right)\right\}_{i=1}^{3}$, in contrast to the infinitesimal generators $\left\{\mathrm{d} \mathcal{U}\left(A_{i}\right)\right\}_{i=1}^{3}$ in the signal domain, do not suffer from interference between these patches.

In literature it is common to either restrict the transform to the sections $s=-\frac{p q}{2}$ or $s=\frac{p q}{2}$ and then the Gabor transform as a function from $\mathbb{R}^{2}$ to $\mathbb{C}$. From a modeling point of view this last consideration is inconsistent, since this violates the uncertainty principle between position and frequency. On $\mathbb{R}^{2}$ the left-invariant vector fields are $\partial_{p}$ and $\partial_{q}$ and they commute $\left[\partial_{p}, \partial_{q}\right]_{\mathbb{R}^{2}}=0$ whereas on the Heisenberg group $\left[\partial_{p}, \partial_{q}\right]=-\partial_{s}$. This theoretic observation has also been reported by Daudet et al.[9]. Moreover, as both sections are plausable, the question rises whether the choice of section matters for the algorithms in literature. In fact, the algorithm is independent on the choice of section if it preserves the phase at each fixed location. In literature this is known as phase invariance. However, this means that one may as well apply a corresponding operator on the amplitude of a Gabor transform, which is actually independent of $t>0$, and to restore the phase afterwards. This has been overlooked in [9], their proposed re-assigment scheme, does employ the group-structure of $\mathrm{H}_{3}$ but relates to a standard re-assignment approach as proposed by [7]. Later we will also take a different view-point on phase preservation, rather than maintaining the phase at each position, we will send the phase along the characteristic curves of our left-invariant evolution equations. We will call this kind of phase preservation phase covariance.

To get a periodic phase in the domain of our Gabor transform we consider $H_{3} / C$ where $C=$ $\{0\} \times\{0\} \times \mathbb{Z}$ is the center of the group $H_{3}$. As the center is a normal subgroup of $H_{3}$ the quotient $H_{3} / C$ is again a group known as the reduced Heisenberg group $H_{r}$. Now the representation $\mathcal{U}^{\lambda}$ given by (2.15) is a representation of the reduced Heisenberg group if and only if $\lambda=n \in \mathbb{Z} \backslash\{0\}$. In the identification of $H_{r} \equiv \mathbb{R}^{2} \times \mathbb{T}, z=e^{2 \pi i s}$, one obtains $\mathcal{U}_{p, q, z}^{n} f(\xi-p)=z^{n} e^{2 \pi i n\left(q \xi-\frac{p q}{2}\right)}$. Consequently we have $\mathcal{W}_{\psi}^{n} f(p, q, z)=z^{-n}\left(\mathcal{W}_{\psi}^{n} f\right)(p, q, 1)$ for all $f, \psi \in \mathbb{L}_{2}(\mathbb{R})$.

Definition 2.1. Let $\mathcal{H}_{n}$ denote that space of all complex-valued functions $F$ on $H_{r}$ such that $F(p, q, z)=z^{-n} F(p, q, 1)$ and $F(\cdot, \cdot, z) \in \mathbb{L}_{2}\left(\mathbb{R}^{2}\right)$ for all $z \in \mathbb{T}$, then clearly $\mathcal{W}_{\psi} f \in \mathcal{H}_{n}$ for all $f, \psi \in \mathcal{H}_{n}$.

In fact $\mathcal{H}_{n}$ is the closure of the space $\left\{\mathcal{W}_{\psi} f \mid \psi, f \in \mathbb{L}_{2}(\mathbb{R})\right\}$ in $\mathbb{L}_{2}\left(H_{r}\right)$. The space $\mathcal{H}_{n}$ is both right and left invariant, since:

$$
\mathcal{W}_{\psi}^{n} \circ \mathcal{U}_{g}^{n}=\mathcal{L}_{g} \circ \mathcal{W}_{\psi}^{n}, \quad \mathcal{W}_{\mathcal{U}_{g}^{n} \psi}^{n}=\mathcal{R}_{g} \circ \mathcal{W}_{\psi}^{n},
$$

where $\mathcal{R}$ denotes the right regular representation on $\mathbb{L}_{2}\left(H_{r}\right)$ and $\mathcal{L}$ denotes the left regular representation on $\mathbb{L}_{2}\left(H_{r}\right)$, i.e.

$$
\mathcal{R}_{g} \phi(h)=\phi(h g) \text { and } \mathcal{L}_{g} \phi(h)=\phi\left(g^{-1} h\right)
$$


for almost every $h, g \in H_{r}$ and every $\phi \in \mathbb{L}_{2}\left(H_{r}\right)$. Since $\mathcal{H}_{n} \subset \mathbb{L}_{2}\left(H_{r}\right)$ we define the right and left regular representations on the space $\mathcal{H}_{n}$ simply by restriction

$$
\mathcal{R}_{g}^{(n)}=\left.\mathcal{R}_{g}\right|_{\mathcal{H}_{n}} \text { and } \mathcal{L}_{g}^{(n)}=\left.\mathcal{R}_{g}\right|_{\mathcal{H}_{n}}
$$

for all $g \in H_{r}$. Now we can identify $\mathcal{H}_{n}$ with $\mathbb{L}_{2}\left(\mathbb{R}^{2}\right)$ by means of the following operator $\mathcal{S}: \mathcal{H}_{n} \rightarrow$ $\mathbb{L}_{2}\left(\mathbb{R}^{2}\right)$ given by

$$
(\mathcal{S} F)(p, q)=F\left(p, q, e^{-2 \pi i \frac{p q}{2}}\right)=e^{i \pi n p q} F(p, q, 1) .
$$

Clearly, this operator is invertible and its inverse is given by

$$
\left(\mathcal{S}^{-1} F\right)(p, q, z)=z^{-n} e^{-i \pi n p q} F(p, q)
$$

Here we recall that $z=e^{2 \pi i s}$ so that this operator simply corresponds to taking the section $s(p, q)=-\frac{p q}{2}$ in the left cosets $H_{3} / \Theta$ where $\Theta$ denotes the phase subgroup $T=\{(0,0, s) \mid s \in \mathbb{R}\}$ of $H_{3}$. We define the following Gabor transform

$$
\mathcal{G}_{\psi}^{n}(f)(p, q)=\mathcal{W}_{\psi}^{\lambda=n}(f)\left(p, q, e^{2 \pi i\left(-\frac{p q}{2}\right)}\right)=\int_{\mathbb{R}} f(\xi) \overline{\psi(\xi-p)} e^{-2 \pi n i(\xi-p) q} \mathrm{~d} \xi,
$$

or briefly $\mathcal{G}_{\psi}^{n}=\mathcal{S} \circ \mathcal{W}_{\psi}^{n}$. To this end we note that in section 6 and section 7 we will consider the evolution equations both on $\mathcal{W}_{\psi}(f)$ defined on the full group $H_{3}$ and on $G_{\psi}(f)$ defined on phase space, where we always keep track of the correspondence using the conjugation with $\mathcal{S}$. To this end we first consider the left and right regular action on the phase space by the left and right regular action on $H_{r}$ and conjugation with $\mathcal{S}^{-1}$. So we define

$$
\tilde{\mathcal{R}}_{g}^{(n)}:=\mathcal{S} \circ \mathcal{R}_{g}^{(n)} \circ \mathcal{S}^{-1}, \quad \tilde{\mathcal{L}}_{g}^{(n)}:=\mathcal{S} \circ \mathcal{L}_{g}^{(n)} \circ \mathcal{S}^{-1} .
$$

A brief computation yields

$$
\begin{aligned}
\tilde{\mathcal{R}}_{g}^{(n)} \phi\left(p^{\prime}, q^{\prime}\right) & =\left(\left(\mathcal{S} \circ \mathcal{R}_{g}^{n}\right)\left((\tilde{p}, \tilde{q}, \tilde{z}) \mapsto(\tilde{z})^{-n} e^{-i n \pi \tilde{p} \tilde{q}} \phi(\tilde{p}, \tilde{q})\right)\left(p^{\prime}, q^{\prime}\right)\right. \\
& =\left(\mathcal{S}\left((\tilde{p}, \tilde{q}, \tilde{z}) \mapsto\left(\tilde{z} z e^{-i \pi(\tilde{p} q-p \tilde{q})}\right)^{-n} e^{-i n \pi(p+\tilde{p})(q+\tilde{q})} \phi(p+\tilde{p}, q+\tilde{q})\right)\right)\left(p^{\prime}, q^{\prime}\right) \\
& =z^{-n} e^{i n \pi\left(-2 p q^{\prime}-p q\right)} \phi\left(p+p^{\prime}, q+q^{\prime}\right) \text { and } \\
\tilde{\mathcal{L}}_{g}^{(n)} \phi\left(p^{\prime}, q^{\prime}\right) & =\left(\mathcal{S}\left((\tilde{p}, \tilde{q}, \tilde{z}) \mapsto\left(\tilde{z} z^{-1} e^{-i \pi(\tilde{p} q-p \tilde{q})}\right)^{-n} e^{-i n \pi(p-\tilde{p})(q-\tilde{q})} \phi(p-\tilde{p}, q-\tilde{q})\right)\right)\left(p^{\prime}, q^{\prime}\right) \\
& =z^{n} e^{n \pi i\left(2 q p^{\prime}-p q\right)} \phi\left(p^{\prime}-p, q^{\prime}-q\right)
\end{aligned}
$$

for all $g=\left(p, q, z=e^{2 \pi i s}\right) \in H_{r}$ and all $\phi \in \mathbb{L}_{2}\left(\mathbb{R}^{2}\right)$.

From the fundamental identities (2.18) we also deduce (by taking the adjoint on both sides of the equality and using the fact that $\mathcal{R}$ and $\mathcal{U}^{\lambda}$ are unitary representations :

$$
\mathcal{U}_{g}^{n} \circ\left(\mathcal{W}_{\psi}^{n}\right)^{*}=\left(\mathcal{W}_{\psi}^{n}\right)^{*} \circ \mathcal{L}_{g} \text {, and }\left(\mathcal{W}_{\psi}^{n}\right)^{*}=\left(\mathcal{W}_{\mathcal{U}_{g} \psi}^{n}\right)^{*} \mathcal{R}_{g}
$$

for all $g \in H_{r}$ and all $n \in \mathbb{Z}$. Now (2.18) and (2.20) imply the following important relation

$$
\begin{aligned}
& \forall_{g \in H_{r}}: \Phi \circ \mathcal{L}_{g}=\mathcal{L}_{g} \circ \Phi \Leftrightarrow \forall_{g \in H_{r}}: \mathcal{U}_{g} \circ \Upsilon_{\psi}=\Upsilon_{\psi} \circ \mathcal{U}_{g}, \\
& \forall_{g \in H_{r}}: \Phi \circ \mathcal{R}_{g}=\mathcal{R}_{g} \circ \Phi \Leftrightarrow \forall_{g \in H_{r}}: \Upsilon_{\mathcal{U}_{g} \psi}=\Upsilon_{\psi} .
\end{aligned}
$$

between operators $\Phi: \mathcal{H}_{n} \rightarrow \mathbb{L}_{2}\left(H_{r}\right)$ on Gabor transforms and corresponding operators

$$
\Upsilon_{\psi}:=\left(\mathcal{W}_{\psi}^{n}\right)^{*} \circ \Phi \circ \mathcal{W}_{\psi}^{n}: \mathbb{L}_{2}(\mathbb{R}) \rightarrow \mathbb{L}_{2}(\mathbb{R})
$$

on signals. So in order to achieve a net modulation and translation covariant operator $\Upsilon_{\psi}$ the operator in the Gabor domain $\Phi$ must be left invariant. Moreover, since $\Upsilon_{\mathcal{U}_{g} \psi}=\Upsilon_{\psi}$ is a highly undesirable property, we do not want the operator $\Phi$ to be right invariant ! 
Theorem 2.2. Let the operator $\Phi$ map the closure $\mathcal{H}_{n}, n \in \mathbb{Z}$, of the space of Gabor transforms into itself, i.e. $\Phi: \mathcal{H}_{n} \rightarrow \mathcal{H}_{n}$. Let $\tilde{\Phi}:=\mathcal{S} \circ \Phi \circ \mathcal{S}^{-1}$ be the corresponding operator on $\mathbb{L}_{2}\left(\mathbb{R}^{2}\right)$ and

$$
Y_{\psi}=\left(\mathcal{W}_{\psi}^{n}\right)^{*} \circ \Phi \circ \mathcal{W}_{\psi}^{n}=\left(\mathcal{S} \mathcal{W}_{\psi}^{n}\right)^{-1} \circ \tilde{\Phi} \circ \mathcal{S} \mathcal{W}_{\psi}^{n}=\left(\mathcal{G}_{\psi}^{n}\right)^{*} \circ \tilde{\Phi} \circ \mathcal{G}_{\psi}^{n}
$$

Then one has the following correspondence:

$$
\Upsilon_{\psi} \circ \mathcal{U}^{n}=\mathcal{U}^{n} \circ \Upsilon_{\psi} \Leftrightarrow \Phi \circ \mathcal{L}^{n}=\mathcal{L}^{n} \circ \Phi \Leftrightarrow \tilde{\Phi} \circ \tilde{\mathcal{L}}^{n}=\tilde{\mathcal{L}}^{n} \circ \tilde{\Phi} .
$$

Proof. More explicitly formulated we have

$$
\forall_{g \in H_{r}}: \Upsilon_{\psi} \circ \mathcal{U}_{g}^{n}=\mathcal{U}_{g}^{n} \circ \Upsilon_{\psi} \Leftrightarrow \forall_{g \in H_{r}}: \Phi \circ \mathcal{L}_{g}^{n}=\mathcal{L}_{g}^{n} \circ \Phi \Leftrightarrow \forall_{g \in H_{r}}: \tilde{\Phi} \circ \tilde{\mathcal{L}}_{g}^{n}=\tilde{\mathcal{L}}_{g}^{n} \circ \tilde{\Phi},
$$

where $Y_{\psi}: \mathbb{L}_{2}\left(\mathbb{R}^{2}\right) \rightarrow \mathbb{L}_{2}\left(\mathbb{R}^{2}\right)$ is the operator on the space of images, $\Phi: \mathcal{H}_{n} \rightarrow \mathcal{H}_{n}$ the corresponding operator on the space of Gabor transforms and $\tilde{\Phi}:=\mathcal{S} \circ \Phi \circ \mathcal{S}^{-1}: \mathbb{L}_{2}\left(\mathbb{R}^{2}\right) \rightarrow \mathbb{L}_{2}\left(\mathbb{R}^{2}\right)$ the corresponding operator on phase space. Now the first equivalence is directly apparent from (2.21), simply by restriction to $\mathcal{H}_{n}$. Furthermore we have by definition of $\tilde{\Phi}$ and $\tilde{\mathcal{L}}^{n}$ that $\tilde{\Phi} \circ \tilde{\mathcal{L}}^{n}=$ $\mathcal{S} \circ \Phi \circ \mathcal{L}^{n} \circ \mathcal{S}^{-1}, \tilde{\mathcal{L}}^{n} \circ \tilde{\Phi}=\mathcal{S} \circ \mathcal{L}^{n} \circ \Phi \circ \mathcal{S}^{-1}$. So the second equivalence follows by the invertibility of (conjugation with) $\mathcal{S}$.

\subsection{Left-invariant vector fields in the Gabor domain and in phase space}

Although Theorem 2.2 is rather simple, it has great practical consequences. For example we would like to find all left-invariant vector fields (considered as first order differential operators) as unbounded operators on both $\mathbb{L}_{2}\left(H_{r}\right)$ (the space in which we embed the Gabor transforms $R\left(\mathcal{W}_{\psi}\right):=\left\{\mathcal{W}_{\psi} f \mid f \in \mathbb{L}_{2}(\mathbb{R})\right\}$ and on $\mathbb{L}_{2}\left(\mathbb{R}^{2}\right)$ (the space in which we embed the phase space restrictions of the Gabor transforms $\left.R\left(\mathcal{G}_{\psi}\right):=\left\{\mathcal{G}_{\psi} f \mid f \in \mathbb{L}_{2}(\mathbb{R})\right\}\right)$. These operators are to be considered as unbounded operators with a domain which is a sufficiently high order Sobolev space such that point evaluation is continuous. In principle one may also assume $\psi$ to be infinitely smooth and rapidly decaying at infinity, in which case the Gabor transform is also infinitely smooth and rapidly decaying.

A field $X$ on a group $G$ is called left-invariant if and only if $X_{g}=L_{g}^{*} X_{e}$ for all $g \in G$, where $L_{g}^{*}$ is the push-forward of the left-multiplication. The left-invariant vector fields are found by means of the derivative of the right-regular representation ${ }^{2}$, which is a Lie-algebra isomorphism between $T_{e}(G)$ equipped with Lie-bracket $(2.11)$ and the Lie-algebra $\mathcal{L}(G)$ of left-invariant vector fields equipped with Lie-product $[\mathcal{A}, \mathcal{B}]=\mathcal{A B}-\mathcal{B} \mathcal{A}$.

Now consider the case $G=H_{3}$ and take the basis $\left\{A_{1}, A_{2}, A_{3}\right\}=\left\{\partial_{p}, \partial_{q}, \partial_{s}\right\}$ for the Liealgebra $T_{e}\left(H_{3}\right)$. Then brief computations yield the left-invariant vector fields $\left\{\mathcal{A}_{1}, \mathcal{A}_{2}, \mathcal{A}_{3}\right\}=$ $\left\{\mathrm{d} \mathcal{R}\left(A_{1}\right), \mathrm{d} \mathcal{R}\left(A_{2}\right), \mathrm{d} \mathcal{R}\left(A_{3}\right)\right\}$, which are given by

$$
\begin{aligned}
& \left.\mathcal{A}_{1}\right|_{g} \phi:=\mathrm{d} \mathcal{R}\left(A_{1}\right) \phi(g)=\lim _{\epsilon \rightarrow 0} \frac{\phi\left(g e^{\epsilon A_{1}}\right)-\phi(g)}{\epsilon}=\left(\left(\partial_{p}+\frac{q}{2} \partial_{s}\right) \phi\right)(g) \\
& \left.\mathcal{A}_{2}\right|_{g} \phi:=\mathrm{d} \mathcal{R}\left(A_{2}\right) \phi(g)=\lim _{\epsilon \rightarrow 0} \frac{\phi\left(g e^{\epsilon A_{2}}\right)-\phi(g)}{\epsilon}=\left(\left(\partial_{q}-\frac{p}{2} \partial_{s}\right) \phi\right)(g) \\
& \left.\mathcal{A}_{3}\right|_{g} \phi:=\mathrm{d} \mathcal{R}\left(A_{3}\right) \phi(g)=\lim _{\epsilon \rightarrow 0} \frac{\phi\left(g e^{\epsilon \epsilon 3}\right)-\phi(g)}{\epsilon}=\partial_{s} \phi(g),
\end{aligned}
$$

for all $g=\left(p, q, e^{2 \pi i s}\right) \in H_{r}$ and all locally defined smooth functions $\phi: \Omega_{g} \subset H_{r} \rightarrow \mathbb{C}$.

Now the practical advantage of using these generators rather than the generators (2.16) is that in the Gabor transform (which is to be considered as score of local frequencies) local grouporbits are torn apart. If for example at a certain position two frequency modulations take place they provide separate responses in the Gabor domain and at both locations the directions of the left-invariant vector fields are well-defined!

Application of these left-invariant vector fields to a Gabor transform, correspond to application of the generators (2.16) to the kernel:

$$
\mathrm{d} \mathcal{R}\left(A_{i}\right)\left(\mathcal{W}_{\psi}(f)\right)(g)=\lim _{\epsilon \rightarrow 0}(1 / \epsilon)\left(\mathcal{U}_{g e^{\epsilon A_{i}}} \psi-\mathcal{U}_{g} \psi, f\right)=\left(\mathcal{U}_{g} \mathrm{~d} \mathcal{U}\left(A_{i}\right) \psi, f\right)=\mathcal{W}_{\mathrm{d} \mathcal{U}\left(A_{i}\right) \psi} f(g) .
$$

\footnotetext{
${ }^{2}$ The right-regular representation is left-invariant whereas the left-regular representation is not left-invariant on a non-commutative group.
} 
From now on we omit the $n$-index. The corresponding operators on phase space are

$$
\left\{\tilde{\mathcal{A}}_{1}, \tilde{\mathcal{A}}_{2}, \tilde{\mathcal{A}}_{3}\right\}=\left\{\mathrm{d} \tilde{\mathcal{R}}\left(A_{1}\right), \mathrm{d} \tilde{\mathcal{R}}\left(A_{2}\right), \mathrm{d} \tilde{\mathcal{R}}\left(A_{3}\right)\right\}=\left\{\mathcal{S} \mathcal{A}_{1} \mathcal{S}^{-1}, \mathcal{S A}_{2} \mathcal{S}^{-1}, \mathcal{S} \mathcal{A}_{3} \mathcal{S}^{-1}\right\}
$$

Straightforward computations yield

$$
\begin{aligned}
& \mathrm{d} \tilde{\mathcal{R}}\left(A_{1}\right) U\left(p^{\prime}, q^{\prime}\right)=\lim _{\epsilon \rightarrow 0} \frac{e^{-i n \pi 2 q^{\prime} \epsilon} U\left(\epsilon+p^{\prime}, q^{\prime}\right)-U\left(p^{\prime}, q^{\prime}\right)}{\epsilon}=\left(\left(\partial_{p^{\prime}}-2 n \pi i q^{\prime}\right) U\right)\left(p^{\prime}, q^{\prime}\right), \\
& \mathrm{d} \tilde{\mathcal{R}}\left(A_{2}\right) U\left(p^{\prime}, q^{\prime}\right)=\lim _{\epsilon \rightarrow 0} \frac{U\left(p^{\prime}, q^{\prime}+\epsilon\right)-U\left(p^{\prime}, q^{\prime}\right)}{\epsilon}=\left(\partial_{q^{\prime}} U\right)\left(p^{\prime}, q^{\prime}\right), \\
& \mathrm{d} \tilde{\mathcal{R}}\left(A_{3}\right) U\left(p^{\prime}, q^{\prime}\right)=-2 i n \pi U\left(p^{\prime}, q^{\prime}\right) .
\end{aligned}
$$

for all $(p, q) \in \mathbb{R}$ and all locally defined smooth functions $U: \Omega_{(p, q)} \subset \mathbb{R}^{2} \rightarrow \mathbb{C}$.

Throughout this article we shall often use the dual left-invariant vector fields. The dual leftinvariant vector fields are spanned by the dual basis

$$
\left.\mathrm{d} \mathcal{A}^{1}\right|_{g=(p, q, s)}=\mathrm{d} p,\left.\quad \mathrm{~d} \mathcal{A}^{2}\right|_{g=(p, q, s)}=\mathrm{d} q,\left.\quad \mathrm{~d} \mathcal{A}^{3}\right|_{g=(p, q, s)}=\mathrm{d} s-\frac{1}{2}(p \mathrm{~d} q-q \mathrm{~d} p),
$$

for all $g \in H_{r}$.

Remark 2.3. These co-vectors are expressed in the co-vectors with respect to the fixed coordinates $\{p, q, s\}$, so that they satisfy

$$
\left\langle\mathrm{d} \mathcal{A}^{i}, \mathcal{A}_{j}\right\rangle=\delta_{j}^{i}, \text { for } i, j=1,2,3 .
$$

Note that $\mathcal{A}_{i}$ is a vector, so the $\mathrm{d}$ within the co-vector $\mathrm{d} \mathcal{A}^{i}$ should not be mistaken for an exterior derivative. So $\mathrm{d} \mathcal{A}^{i}$ is just a notation for corresponding dual basis, defined by (2.25).

\subsection{The Cauchy Riemann equations on Gabor transforms}

If $\psi(\xi)=e^{-\pi n \xi^{2}}$ and $f$ is some arbitrary signal in $\mathbb{L}_{2}(\mathbb{R})$ then the Gabor transform $\mathcal{W}_{\psi}(f)$ satisfies

$$
\psi(\xi)=e^{-\pi n \xi^{2}} \Rightarrow\left(\mathcal{A}_{2}+i \mathcal{A}_{1}\right) \mathcal{W}_{\psi}(f)=0
$$

Equivalently, we find by application of $\mathcal{S}$ that the Gabor transform $\mathcal{G}_{\psi} f$ satisfies

$$
\psi(\xi)=e^{-\pi \xi^{2} n} \Rightarrow\left(\tilde{\mathcal{A}}_{2}+i \tilde{\mathcal{A}}_{1}\right) \mathcal{G}_{\psi}(f)=0,
$$

where we recall that $\mathcal{G}_{\psi}(f)=\mathcal{S} \mathcal{W}_{\psi}(f)$ and $\mathcal{A}_{i}=\mathcal{S}^{-1} \tilde{\mathcal{A}}_{i} \mathcal{S}$ for $i=1,2,3$. Equality (2.27) on phase space has been reported previously by [9].

For analysis (not for fast implementation) the equivalent equation on the group (2.26) is much more tangible, because the left-invariant vector fields on $H_{r}$ are proper derivatives, in contrast to the left invariant vector fields on phase-space $\mathbb{R}^{2}$ where the time derivative in the first generator is replaced by a cumbersome multiplication operator.

Recall to this end from classical function theory that the logarithm of an analytic function is again analytic, which directly follows from the identity: $\partial_{\bar{z}} \log f(z, \bar{z})=\frac{\partial_{\bar{z}} f(z, \bar{z})}{f(z, \bar{z})}$, with $z=x+i y$ and $\partial_{\bar{z}}=\partial_{x}+i \partial_{y}$. Now the logarithm yields a decomposition in local phase and localamplitude, so apparently there exist Cauchy-Riemann relations between derivations on local phase and local amplitude. This is often very useful in signal processing since these relations allow us to relate local phase derivatives by amplitude derivatives, where the local amplitude does not suffer from branch-cuts.

Definition 2.4. A smooth complex-valued function $f$ on $\mathbb{C}$ is analytic iff $\left(\partial_{x}+i \partial_{y}\right) f(x+i y)=0$, a complex-valued smooth function $U$ on ${ }^{3} H_{r}$ is analytic iff $\left(\mathcal{A}_{2}+i \mathcal{A}_{1}\right) U=0$.

\footnotetext{
${ }^{3}$ To be more precise a function on the non-integrable contact manifold $\left(H_{r}, \mathrm{~d} \mathcal{A}_{3}\right)$ within $H_{r}$. For further explanation see Appendix B or for general theory on contact manifolds cf. [4].
} 
Remark 2.5. There is a big difference between analyticity of functions on $\mathbb{C}$ and analyticity of functions on $H_{r}$. In Definition 2.4 operators $\partial_{x}$ and $\partial_{y}$ are two (left)-invariant vector fields on the group $\mathbb{R}^{2}$ and $\mathcal{A}_{1}$ and $\mathcal{A}_{2}$ are left-invariant vector fields on $H_{r}$. However the vector fields $\mathcal{A}_{1}, \mathcal{A}_{2}$ do not commute and thereby they form a non-integrable foliation in $H_{r}$. Consequently, fundamental theorems like the Liouville theorem on analytic functions on $\mathbb{R}^{2}$ do not directly apply to analytic functions on $H_{r}$. For example for suitable choice of $\psi$ the Gabor transform is both bounded and analytic on $H_{r}$. The property, that the logarithm of an analytic function is again analytic, however, does naturally generalize to $H_{r}$ since it only uses the chain-rule for differentiation with respect to the fixed coordinate system $(p, q, s)$.

Now using the chainrule for differentiation we directly deduce from (2.26) that

$$
\begin{aligned}
& \left(\mathcal{A}_{2}+i \mathcal{A}_{1}\right) \mathcal{W}_{\psi}(f)=0 \Leftrightarrow\left(\mathcal{A}_{2}+i \mathcal{A}_{1}\right) \log \left(\mathcal{W}_{\psi}(f)\right)=0 \\
& \Leftrightarrow\left(\mathcal{A}_{2}+i \mathcal{A}_{1}\right)\left(\log \left|\mathcal{W}_{\psi}(f)\right|+i \arg \left\{\mathcal{W}_{\psi}(f)\right\}\right)=0 \\
& \Leftrightarrow|U| \mathcal{A}_{2} \Omega=-\mathcal{A}_{1}|U|=-\partial_{p}|U| \text { and }|U| \mathcal{A}_{1} \Omega=\mathcal{A}_{2}|U|=\partial_{q}|U|,
\end{aligned}
$$

where we use short notation $U=\mathcal{W}_{\psi}(f)$ and $\Omega=\arg \left\{\mathcal{W}_{\psi}(f)\right\}$.

For the corresponding equations on phase space one must be careful (a simple replacement $\mathcal{A}_{i} \rightarrow \tilde{\mathcal{A}}_{i}$ will not do because the multiplication operator $2 \pi q i$, in contrast to $\partial_{s}$ does not satisfy the chainrule). A brief computation yields

$$
\left(\tilde{\mathcal{A}}_{2}+i \tilde{\mathcal{A}}_{1}\right) \mathcal{G}_{\psi}(f)=0 \Rightarrow|\tilde{U}| \tilde{\mathcal{A}}_{2} \tilde{\Omega}=-\mathcal{A}_{1}|\tilde{U}|=-\partial_{p}|\tilde{U}| \text { and }|\tilde{U}| \tilde{\mathcal{B}}_{1} \tilde{\Omega}=\mathcal{A}_{2}|\tilde{U}|=\partial_{q}|\tilde{U}|
$$

where we use short notation $\tilde{U}=\mathcal{G}_{\psi}(f)$ and $\tilde{\Omega}=\arg \left\{\mathcal{G}_{\psi}(f)\right\}$ and where

$$
\tilde{B}_{1} \tilde{\Omega}=\partial_{p} \tilde{\Omega}-2 \pi q .
$$

Now operator $\tilde{B}_{1}$ is not a left-invariant vector field. However, the composition operator $\tilde{B}_{1} \circ \arg$ is left invariant.

Sofar we had to consider the from a practical point of view not so interesting case $a=1$ gives $\psi(\xi)=e^{-\pi n(\xi-c)^{2}}, c>0$. However, a solution in the general case is simply obtained by dilation

$$
\psi_{a}(\xi)=e^{-a^{-2} \pi n(\xi-c)^{2}} \text { with } a>0, c>0,
$$

where $c>0$ is a shift parameter and $a>0$ a scaling parameter. Next we briefly motivate our scaling argument. It is easily verified that the Gabor transform admits the following scaling relation:

$$
\mathcal{G}_{\psi_{a}} f(p, q)=\sqrt{a} \mathcal{G}_{\mathcal{D}_{a} \psi}(f)(p, q)=\sqrt{a} \mathcal{G}_{\psi} \mathcal{D}_{a} f\left(\frac{p}{a}, a q\right)
$$

where $\psi=\psi_{a=1}$ and where the unitary dilation operator $\mathcal{D}_{a}: \mathbb{L}_{2}(\mathbb{R}) \rightarrow \mathbb{L}_{2}(\mathbb{R})$ is given by $\mathcal{D}_{a}(\psi)(x)=a^{-\frac{1}{2}} f(x / a), a>0, f \in \mathbb{L}_{2}(\mathbb{R})$. Now we have

$$
\begin{aligned}
& \forall_{f \in \mathbb{L}_{2}(\mathbb{R})} \forall_{p^{\prime}, q^{\prime} \in \mathbb{R}}\left(i\left(\partial_{p^{\prime}}-2 \pi i q^{\prime}\right)+\partial_{q^{\prime}}\right)\left(\mathcal{G}_{\psi}\left(\mathcal{D}_{\frac{1}{a}} f\right)\right)\left(p^{\prime}, q^{\prime}\right)=0 \Leftrightarrow \\
& \forall_{f \in \mathbb{L}_{2}(\mathbb{R})} \forall_{p, q \in \mathbb{R}}\left(i\left(a \partial_{p}-2 \pi i a q\right)+\frac{1}{a} \partial_{q}\right)\left(\mathcal{G}_{\psi_{a}}(f)(p, q)=0,\right.
\end{aligned}
$$

which simply follows by substitution $q^{\prime}=a q, p^{\prime}=p / a$. So if we define

$$
\tilde{\mathcal{A}}_{1}^{a}:=a \tilde{\mathcal{A}}_{1} \text { and } \tilde{\mathcal{A}}_{2}^{a}:=a^{-1} \tilde{\mathcal{A}}_{2},
$$

then we have for all signals $f \in \mathbb{L}_{2}(\mathbb{R})$ that

$$
\left(\tilde{\mathcal{A}}_{2}^{a}+i \tilde{\mathcal{A}}_{1}{ }^{a}\right) \mathcal{G}_{\psi}(f)=0 \text { and }\left(\mathcal{A}_{2}^{a}+i \mathcal{A}_{1}^{a}\right) \mathcal{W}_{\psi}(f)=0 .
$$

and consequently,

$$
\begin{array}{lll}
\left|\tilde{U}^{a}\right| \partial_{q} \tilde{\Omega}^{a}=-a^{2} \partial_{p}\left|\tilde{U}^{a}\right| & \text { and } & \left|\tilde{U}^{a}\right| \partial_{p} \tilde{\Omega}^{a}=a^{-2} \partial_{q}\left|\tilde{U}^{a}\right|+2 \pi q . \\
\mathcal{A}_{2} \Omega^{a}=a^{2} \mathcal{A}_{1}\left|U^{a}\right| & \text { and } & \mathcal{A}_{1} \Omega^{a}=a^{-2} \mathcal{A}_{1}\left|U^{a}\right|
\end{array}
$$

where $\tilde{U}^{a}$ respectively $U$ is short notation for $\tilde{U}^{a}=\mathcal{G}_{\psi_{a}}(f)$ and $U^{a}=\mathcal{W}_{\psi_{a}}(f)$ and $\tilde{\Omega}^{a}=$ $\arg \left\{\mathcal{G}_{\psi_{a}}(f)\right\}$ and $\Omega^{a}=\arg \left\{\mathcal{W}_{\psi_{a}}(f)\right\}$. 


\subsection{Gabor transform and inverse Fourier transform on $H_{r}$}

The unitarity of the Gabor transform directly follows from the Fourier transform on $H_{r}$, see Appendix A. The set $\left\{\mathcal{U}^{\lambda=n}\right\}_{n \in \mathbb{Z}}$ is up to equivalence the unique set of unitary irreducible representations of $H_{r}$. We can now express the Gabor transform as the inverse Fourier transform of a function $A_{f, \psi}: \widehat{H_{r}} \rightarrow H\left(\mathbb{L}_{2}(\mathbb{R})\right)$, where $H\left(\mathbb{L}_{2}(\mathbb{R})\right)$ denotes the space of trace-class operators on $\mathbb{L}_{2}(\mathbb{R})$ :

$$
\mathcal{W}_{\psi}^{n} f\left(g^{-1}\right)=\left(\psi,\left(\mathcal{U}_{g^{-1}}^{n}\right)^{*} f\right)=\operatorname{trace}\left(f \otimes \psi \circ\left(\mathcal{U}_{g}^{n}\right)\right)=\frac{1}{\nu_{H_{r}}(\{\mathcal{U}\})} \int_{\hat{H}_{r}} \operatorname{trace}\left\{A_{f, \psi}(\sigma)(\sigma(g))^{*}\right\} \mathrm{d} \nu_{\bar{H}_{r}}(\sigma)
$$

where $\nu_{\widehat{H_{r}}}$ denotes the Plancherel measure on the dual group $\widehat{H_{r}}$

$$
A_{f, \psi}(\sigma)= \begin{cases}0 & \text { if } \sigma \neq \mathcal{U}^{n} \\ f \otimes \psi & \text { if } \sigma=\mathcal{U}^{n}\end{cases}
$$

So indeed application of the Plancherel Theorem yields

$$
\left\|\mathcal{W}_{\psi}(f)\right\|^{2}=\int_{\widehat{H}_{r}} \frac{1}{\left(\nu_{\widehat{H_{r}}}\left(\mathcal{U}^{n}\right)\right)^{2}}\left\|A_{f, \psi}(\sigma)\right\|^{2} \mathrm{~d} \nu_{\widehat{H_{r}}}(\sigma)=\frac{1}{\nu_{\widehat{H_{r}}}\left(\left\{\mathcal{U}^{n}\right\}\right)}\|f\|^{2}\|\psi\|^{2}
$$

where we note that the Hilbert Schmidt norm of $\|f \otimes \psi\|=\|f\|\|\psi\|$. So the constant $C_{\psi}$ in (1.4) actually equals $C_{\psi}=\frac{\|\psi\|^{2}}{\nu_{\widehat{H_{r}}}\left(\left\{\mathcal{U}^{\lambda=n}\right\}\right)}$.

More explicitly we have $\nu_{\widehat{H}_{r}}\left(\left\{\mathcal{U}^{\lambda=n}\right\}\right)=n$, recall (1.4), and if we again use the identification $n \leftrightarrow \mathcal{U}^{n}$ (like in Appendix A) we get

$$
\begin{aligned}
\mathcal{W}_{\psi}^{n} f\left(g^{-1}\right) & =\operatorname{trace}\left\{f \otimes \psi \circ \mathcal{U}_{g}^{n}\right\} \\
& =\frac{1}{n} \sum_{n^{\prime} \in \mathbb{Z}} n^{\prime} \operatorname{trace}\left\{A_{f, \psi}\left(n^{\prime}\right) \mathcal{U}_{g}^{n^{\prime}}\right\}=\left[\mathcal{F}_{H_{r}}^{-1} A_{f, \psi}\right](g)
\end{aligned}
$$

\section{Discrete Gabor Transforms}

The discrete Gabor transform is given by

$$
\left(\mathcal{W}_{\boldsymbol{\psi}}^{D} \mathbf{f}\right)[l, m, k] \quad:=e^{-2 \pi i\left(\frac{k}{Q}-\frac{m l L}{2 M}\right)} \frac{1}{N} \sum_{n=0}^{N-1} \overline{\psi[n-l L]} \mathbf{f}[n] e^{-\frac{2 \pi i n m}{M}}
$$

with $L, K, N, M, Q \in \mathbb{N}$ and

$$
k=0,1, \ldots, Q-1 \text { and } l=0, \ldots, K-1, m=0, \ldots, M-1, \text { with } L=\frac{N}{K},
$$

and integer oversampling $P=M / L \in \mathbb{Z}$ and where the discrete signal is given by $\mathbf{f}=\{\mathbf{f}[n]\}_{n=0}^{N-1}:=$ $\left\{f\left(\frac{n}{N}\right)\right\}_{n=0}^{N-1} \in \mathbb{R}^{N}$ and where the discrete kernel is given by a sampled Gaussian kernel

$$
\boldsymbol{\psi}=\{\boldsymbol{\psi}[n]\}_{n=-(N-1)}^{N-1}:=\left\{e^{-\frac{\left(|n|-\left\lfloor\frac{N-1}{2}\right\rfloor\right)^{2} \pi}{N^{2} a^{2}}}\right\}_{n=-(N-1)}^{N-1} \in \mathbb{R}^{N}
$$

with $\frac{\pi}{a^{2}}=\frac{1}{2 \sigma^{2}}$ where $\sigma^{2}$ is the variance of the Gaussian. Now we have $P N=M K$, or equivalently

$$
\frac{1}{M}=\frac{K}{P} \frac{1}{N} .
$$

We note that it is important that the discrete kernel is $N$ periodic since from the fact that $N=K L$ it directly follows that

$$
\forall_{\mathbf{f} \in \ell_{2}(I)} \forall_{l, m, k} \mathcal{W}_{\boldsymbol{\psi}}^{D} \mathbf{f}[l+K, m, k]=\mathcal{W}_{\boldsymbol{\psi}}^{D} \mathbf{f}[l, m, k] \Leftrightarrow \forall_{n=0, \ldots N} \boldsymbol{\psi}[n-N]=\boldsymbol{\psi}[n],
$$


where $I=\{0, \ldots, N-1\}$. Moreover, we note that the kernel chosen in (3.36) is even.

Now for Riemann-integrable $f$ with support within $[0,1]$ and $\psi$ even with support within $[-1,1]$, say

$$
\psi(\xi)=e^{-\frac{\pi|| \xi\left|-\frac{1}{2}\right|^{2}}{a^{2}}} 1_{[-1,1]}(\xi),
$$

we have

$$
\begin{aligned}
\left(W_{\boldsymbol{\psi}}^{D} \mathbf{f}\right)[l, m, k] & =\frac{1}{N} e^{-2 \pi i\left(\frac{k}{Q}-\frac{m l L}{2 M}\right)} \sum_{n=0}^{N-1} e^{-\pi a^{-2} \frac{\left(|n-l L|-\left\lfloor\frac{N-1}{2}\right\rfloor\right)^{2}}{N^{2}}} f\left(\frac{n}{N}\right) e^{-\frac{2 \pi i n m}{M}} \\
& =e^{-2 \pi i\left(\frac{k}{Q}-\frac{m l}{2 P}\right)} \frac{1}{N} \sum_{n=0}^{N-1} e^{-\pi a^{-2}\left(\left|\frac{n}{N}-\frac{l}{K}\right|-\frac{1}{N}\left\lfloor\frac{N-1}{2}\right\rfloor\right)^{2}} f\left(\frac{n}{N}\right) e^{-\frac{2 \pi(K / P) i n m}{N}} \\
& \rightarrow e^{-2 \pi i\left(\frac{k}{Q}-\frac{1}{2} \frac{m K}{P} \frac{l}{K}\right)} \int_{0}^{1} f(\xi) e^{-\frac{\pi\left(\left|\xi-\frac{l}{K}\right|-\frac{1}{2}\right)^{2}}{a^{2}}} e^{-2 \pi i \xi\left(\frac{m K}{P}\right)} \mathrm{d} \xi .
\end{aligned}
$$

So that we have

$$
\left(W_{\boldsymbol{\psi}}^{D} \mathbf{f}\right)[l, m, k] \rightarrow \mathcal{W}_{\psi}^{n=1} f\left(p=\frac{l}{K}, q=\frac{m K}{P}, s=\frac{k}{Q}\right) \text { as } N \rightarrow \infty .
$$

where we keep both $P$ and $K$ fixed so that only $M \rightarrow \infty$ as $N \rightarrow \infty$ and where we recall that the continuous Gabor transform was given by

$$
\mathcal{W}_{\psi}^{n=1} f(p, q, s)=e^{-2 \pi i\left(s-\frac{p q}{2}\right)} \int_{\mathbb{R}} \overline{\psi(\xi-p)} f(\xi) e^{-2 \pi i \xi q} \mathrm{~d} \xi,
$$

where we again took the scaled Gaussian kernel $\psi(\xi)=e^{-\pi a^{-2} \xi^{2}} 1_{[-1,1]}(\xi)$ and we took pointwise limit in the reproducing kernel space of Gabor transforms.

\subsection{Diagonalization of the Gabor transform}

In our algorithms, we used the diagonalization of the discrete Gabor transform by means of the discrete Zak-transform. Next we give a brief summary of this diagonalization, for more details we refer to [29] and [27]. The finite frame operator $\mathfrak{F}: \ell_{2}(I) \rightarrow \ell_{2}(I)$, is given by

$$
[\mathfrak{F} \mathbf{f}][n]=\sum_{l=0}^{K-1} \sum_{m=0}^{M-1}\left(\boldsymbol{\psi}_{l m}, \mathbf{f}\right) \boldsymbol{\psi}_{l m}[n], \quad n \in I,
$$

with $\boldsymbol{\psi}_{l m}=\mathcal{U}_{\left[l, m, k=-\frac{Q l m}{2 P}\right]} \boldsymbol{\psi}$. Now there exists Riesz-bounds $A, B>0$ such that $A(f, f) \leq(\mathfrak{F} f, f)$, so $\mathfrak{F}$ is bounded from below and thereby $\mathfrak{F}^{-1}$ exists. Now clearly $\mathfrak{F}^{*}=\mathfrak{F}$ and

$$
\mathbf{f}=\mathfrak{F}^{-1} \mathfrak{F} \mathbf{f}=\sum_{l=0}^{K-1} \sum_{m=0}^{M-1}\left(\boldsymbol{\psi}_{l m}, f\right) \mathfrak{F}^{-1} \boldsymbol{\psi}_{l m},
$$

so we see that $\boldsymbol{\psi}_{l m}$ is the analysis, window and $\mathfrak{F}^{-1} \boldsymbol{\psi}_{l m}$ is the synthesis window. Now we also write

$$
f=\mathfrak{F}^{-\frac{1}{2}} \mathfrak{F} \mathfrak{F}^{-\frac{1}{2}} f=\sum_{l=0}^{K-1} \sum_{m=0}^{M-1}\left(\boldsymbol{\psi}_{l m}, \mathfrak{F}^{-\frac{1}{2}} \mathbf{f}\right) \mathfrak{F}^{-\frac{1}{2}} \boldsymbol{\psi}_{l m}=\sum_{l=0}^{K-1} \sum_{m=0}^{M-1}\left(\mathfrak{F}^{-\frac{1}{2}} \boldsymbol{\psi}_{l m}, \mathbf{f}\right) \mathfrak{F}^{-\frac{1}{2}} \boldsymbol{\psi}_{l m}
$$

and thereby to each frame one can associate a tight frame (i.e. the Riesz-bounds coincide), where dual and synthesis window coincide. Since $\mathfrak{F}$ is self-adjoint on a finite dimensional ${ }^{4}$ vector space it has a orthonormal basis of eigen vectors which are given by

$$
\mathbf{u}_{n k}\left[n^{\prime}\right]=\frac{1}{\sqrt{K}} v\left[n^{\prime}-n\right] e^{\frac{2 \pi i k}{N}\left(n^{\prime}-n\right)}, \text { with } v(n)=\sum_{l=-\infty}^{\infty} \delta[n-l L]
$$

\footnotetext{
${ }^{4}$ In the continuous setting (or in the infinite discrete setting) the frame operator is self-adjoint but unfortunately not compact and one has to work with generalized eigenvectors in a Gelfand-triple.
} 
for $n \in\{0, \ldots, L-1\}, k \in\{0, \ldots, K-1\}$, where we recall $N=K L$. so that we can put the frame operator into diagonal form :

$$
\mathfrak{F}=\left(Z^{D}\right)^{-1} \circ \Lambda \circ Z^{D},
$$

where the Discrete Zak transform is given by $\left[Z^{D} \mathbf{f}\right][n, k]=\left(\mathbf{u}_{n k}, \mathbf{f}\right)_{\ell_{2}(I)}$ so that $\mathfrak{F} \mathbf{f}=\sum_{n=0}^{L-1} \sum_{k=0}^{K-1} \lambda_{n k}\left(\mathbf{u}_{n k}, \mathbf{f}\right) \mathbf{u}_{n k}$, with eigenvalues $\lambda_{n k}=L \sum_{p=0}^{P-1}\left|Z \boldsymbol{\psi}^{D}\left[n, k-p \frac{N}{M}\right]\right|^{2}$ and integer oversampling factor $P=M / L$.

We stress that all these considerations boil down to diagonalization of inverse Fourier transform on the discrete Heisenberg group, where we recall (2.33).

\section{Discrete Left-invariant vector fields}

Similar to the continuous case the discrete Gabor transform can be written

$$
\left[\mathcal{W}_{\boldsymbol{\psi}}^{D} \mathbf{f}\right][l, m, k]=\left(\mathcal{U}_{[l, k, m]} \boldsymbol{\psi}, \mathbf{f}\right)_{\ell_{2}(I)},
$$

where $I=\{0, \ldots, N-1\}$ and $(\mathbf{a}, \mathbf{b})=\frac{1}{N} \sum_{i=0}^{N-1} \bar{a}_{i} b_{i}$.

$$
\mathcal{U}_{[l, k, m]} \boldsymbol{\psi}[n]=e^{2 \pi i\left(\frac{k}{Q}-\frac{m l}{2 P}\right)} e^{\frac{2 \pi i n m}{M}} \boldsymbol{\psi}[n-l L] .
$$

From now on we shall assume $\frac{Q}{2 P} \in \mathbb{Z}, L$ even, $N$ even (reasonable assumptions in practice) so that we can define the following finite reduced Heisenberg group $\mathfrak{h}_{r}=\{[l, m, k] \mid l=0, \ldots, K-1, m=$ $0, \ldots, M-1, k=0, \ldots, Q-1\}$ equipped with product

$$
[l, m, k]\left[l^{\prime}, m^{\prime}, k^{\prime}\right]=\left[l+l^{\prime} \operatorname{Mod} K, m+m^{\prime} \operatorname{Mod} M, k+k^{\prime}+\frac{Q}{2 P}\left(m l^{\prime}-m^{\prime} l\right) \operatorname{Mod}(Q)\right],
$$

which is well-defined as long as $\frac{K}{2 P}=\frac{N}{2 M} \in \mathbb{N}$ :

$$
\begin{aligned}
& {[l+\alpha K, m+\beta M, k+\gamma Q]\left[l^{\prime}, m^{\prime}, k^{\prime}\right]=} \\
& {\left[l+l^{\prime} \operatorname{Mod} \mathrm{K}, m+m^{\prime} \operatorname{Mod~M}, k+k^{\prime}+\frac{Q}{2 P}\left(m l^{\prime}-l m^{\prime}\right)+\frac{Q}{2 P}\left(\beta M l^{\prime}-\alpha K m^{\prime}\right) \operatorname{Mod} Q\right]} \\
& =\left[l+l^{\prime} \operatorname{Mod} \mathrm{K}, m+m^{\prime} \operatorname{Mod} \mathrm{M}, k+k^{\prime}+\frac{Q}{2 P}\left(m l^{\prime}-l m^{\prime}\right) \operatorname{Mod} Q\right], \quad \alpha, \beta, \gamma \in \mathbb{N} .
\end{aligned}
$$

since $\frac{M}{P}=L \in \mathbb{N}$. This group is the domain of the discrete Gabor transforms, since for all $\boldsymbol{\psi} \in \ell_{2}(I)$ we have

$$
\mathcal{U}_{\left[l^{\prime}, k^{\prime}, m^{\prime}\right]} \mathcal{U}_{[l, k, m]} \psi=\mathcal{U}_{\left[l^{\prime}, k^{\prime}, m^{\prime}\right][l, k, m]} \psi
$$

Based on the convergence result (3.39) we define the following mapping

$$
\phi[l, m, k]=\left(\frac{l}{K}, \frac{m K}{P}, \frac{k}{Q}\right)
$$

which sets an monomorphism between the discrete group $h_{r}=\{[l, m, k] \mid l, m, k \in \mathbb{Z}\}$ which is equipped with group product

$$
[l, m, k]\left[l^{\prime}, m^{\prime}, k^{\prime}\right]=\left[l+l^{\prime}, m+m^{\prime}, k+k^{\prime}+\frac{Q}{2 P}\left(m l^{\prime}-m^{\prime} l\right)\right] .
$$

and the continuous Heisenberg group $H_{3}$ :

$$
\begin{aligned}
\phi[l, m, k] \phi\left[l^{\prime}, m^{\prime}, k^{\prime}\right] & =\left(\frac{l}{K}, \frac{m K}{P}, \frac{k}{Q}\right)\left(\frac{l}{K}, \frac{m K}{P}, \frac{k}{Q}\right) \\
& =\left(\frac{l+l^{\prime}}{K}, \frac{\left(m+m^{\prime}\right) K}{P}, \frac{k+k^{\prime}+\frac{Q}{2 P}\left(m l^{\prime}-l m^{\prime}\right)}{Q}\right)=\phi\left[[l, m, k]\left[l^{\prime}, m^{\prime}, k^{\prime}\right]\right] .
\end{aligned}
$$


Here we note that the mapping $\phi$ maps the discrete variables on a uniform grid in the continuous domain:

$$
s \in[0,1) \leftrightarrow k \in[0, Q) \cap \mathbb{Z}, \quad p \in[0,1) \leftrightarrow l \in[0, K) \cap \mathbb{Z}, \quad q \in[0, N) \leftrightarrow m \in[0, M) \cap \mathbb{Z} .
$$

The group $h_{r}$ is not isomorphic to a subgroup of $H_{r}$ since we have periodicity in both $l$ and $m$. However, if again $\frac{N}{2 M} \in \mathbb{N}$, then it equals the quotient $\mathfrak{h}_{r}:=h_{r} /[K \mathbb{Z}, M \mathbb{Z}, Q \mathbb{Z}]$ of the discrete group $h_{r}$ with the normal subgroup $[K \mathbb{Z}, M \mathbb{Z}, Q \mathbb{Z}]$, where we note that $\frac{N}{2 M}$ and $N$ even implies that $[K \mathbb{Z}, M \mathbb{Z}, Q \mathbb{Z}]=\left[K \mathbb{Z}, M \mathbb{Z}, Q \mathbb{Z}-\frac{Q}{2 P} M K \mathbb{Z}\right]($ recall that $M K=P N)$.

Then on this quotient-group $\mathfrak{h}_{r}$ we define the forward left-invariant vector fields on discrete Gabor-transforms as follows (where we again use (3.37) and (3.35)):

$$
\begin{aligned}
& \left(\mathcal{A}_{1}^{D^{+}} W_{\boldsymbol{\psi}}^{D} \mathbf{f}\right)[l, m, k]=K\left(\mathrm{~d} \mathcal{R}^{D^{+}}[1,0,0] W_{\boldsymbol{\psi}}^{D} \mathbf{f}\right)[l, m, k]=\frac{W_{\boldsymbol{\psi}}^{D} \mathbf{f}([l, m, k][1,0,0])-W^{D}}{\boldsymbol{\psi}^{\mathrm{f}[l, m, k]}} \\
& =\frac{e^{-\frac{\pi i m}{P}} W_{\boldsymbol{\psi}}^{D} \mathbf{f}[l+1, m, k]-W^{D} \mathbf{f}[l, m, k]}{K^{-1}}=\frac{e^{-\frac{\pi i m L}{M}} W^{D} \mathbf{f}[l+1, m, k]-W^{D} \boldsymbol{\psi}^{\mathbf{f}[l, m, k]}}{K^{-1}} \\
& \left(\mathcal{A}_{2}^{D^{+}} W_{\boldsymbol{\psi}}^{D} \mathbf{f}\right)[l, m, k]=\frac{M}{N}\left(\mathrm{~d} \mathcal{R}^{D^{+}}[0,1,0] W_{\boldsymbol{\psi}}^{D} \mathbf{f}\right)[l, m, k]=\frac{e^{+\frac{\pi i l}{P}}{ }^{D} \boldsymbol{\psi}^{\mathbf{f}[l, m+1, k]-W^{D}} \boldsymbol{\psi}^{\mathbf{f}[l, m, k]}}{K P^{-1}} \\
& =\frac{e^{+\frac{\pi i l L}{M}} W_{\boldsymbol{\psi}^{\mathrm{f}}[l, m+1, k]-W^{-}}^{D} \mathbf{f}[l, m, k]}{N M^{-1}} \\
& \left(\mathcal{A}_{3}^{D^{+}} W_{\boldsymbol{\psi}}^{D} \mathbf{f}\right)[l, m, k]=Q\left(\mathrm{~d} \mathcal{R}^{D^{+}}[0,0,1] W_{\boldsymbol{\psi}}^{D} \mathbf{f}\right)[l, m, k]=\frac{W_{\boldsymbol{\psi}^{\mathrm{f}}[l, m, k+1]-W^{2}}^{D} \mathbf{f}[l, m, k]}{Q^{-1}} \\
& \left.=Q\left(e^{\frac{-2 \pi i}{Q}}-1\right) W_{\boldsymbol{\psi}}^{D} \mathbf{f}[l, m, k]\right)
\end{aligned}
$$

and the backward discrete left-invariant vector fields

$$
\begin{aligned}
& \left(\mathcal{A}_{1}^{D^{-}} W_{\boldsymbol{\psi}}^{D} \mathbf{f}\right)[l, m, k]=\left(\mathrm{d} \mathcal{R}^{D^{-}}[1,0,0] W_{\boldsymbol{\psi}}^{D} \mathbf{f}\right)[l, m, k] \\
& =\frac{{ }^{D} \boldsymbol{\psi}^{\mathbf{f}[l, m, k]-e^{+\frac{\pi i m L}{M}} W^{D}} \boldsymbol{\psi}^{\mathbf{f}[l-1, m, k]}}{K^{-1}} \\
& \left(\mathcal{A}_{2}^{D^{-}} W_{\boldsymbol{\psi}}^{D} \mathbf{f}\right)[l, m, k]=\left(\mathrm{d} \mathcal{R}^{D^{+}}[0,1,0] W_{\boldsymbol{\psi}}^{D} \mathbf{f}\right)[l, m, k] \\
& =\frac{{ }^{D} \boldsymbol{\psi}^{\mathbf{f}[l, m, k]-e^{-\frac{\pi i l L}{M}}} W_{\boldsymbol{\psi}}^{D} \mathbf{f}[l, m-1, k]}{N M^{-1}} \\
& \left(\mathcal{A}_{3}^{D^{-}} W_{\boldsymbol{\psi}}^{D} \mathbf{f}\right)[l, m, k]=\frac{N M^{-1}}{\left(\mathrm{~d} \mathcal{R}^{D^{+}}[0,0,1] W_{\boldsymbol{\psi}}^{D} \mathbf{f}\right)[l, m, k]}
\end{aligned}
$$

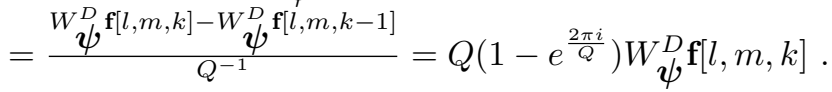

Note that left-invariant central differences are given by the average of a left-invariant forward and left-invariant backward difference.

Remark 4.6. With respect to the denominators (i.e. step-sizes) in (4.43) and (4.44) we recall (3.39) where for the sake of consistent convergence we have set $p=\frac{l}{K}, q=\frac{m}{M} N, \xi=\frac{n}{N}, s=\frac{k}{Q}$, so that the actual discrete steps are $\Delta p=K^{-1}, \Delta q=N M^{-1}$ and $\Delta s=Q^{-1}$.

Just like in the continuous case we can use the following discrete version $S^{D}$ of the operator $\mathcal{S}$ which maps a Gabor transform $W_{\boldsymbol{\psi}}^{D}$ onto its phase space representation $G_{\boldsymbol{\psi}}^{D}$ :

$$
G_{\boldsymbol{\psi}}^{D} \mathbf{f}[l, m]:=\left(S^{D} W_{\boldsymbol{\psi}}^{D}\right) \mathbf{f}[l, m]=W_{\boldsymbol{\psi}}^{D} \mathbf{f}\left[l, m,-\frac{Q l m}{2 P}\right], \quad P=M / L,
$$

and the inverse is given by

$$
W_{\boldsymbol{\psi}}^{D} \mathbf{f}[l, m, k]=\left(\left(S^{D}\right)^{-1} G_{\boldsymbol{\psi}}^{D} \mathbf{f}\right)[l, m, k]=e^{-2 \pi i\left(\frac{k}{Q}+\frac{l m L}{2 M}\right)} G_{\boldsymbol{\psi}^{D}}^{D} \mathbf{f}[l, m]
$$

Again we can use the conjugation with $S^{D}$ to map the left-invariant discrete vector fields $\left\{\mathcal{A}_{i}^{D^{ \pm}}\right\}_{i=1}^{3}$ to the corresponding discrete vector fields on the discrete phase space: $\tilde{\mathcal{A}}_{i}^{D^{ \pm}}=\left(S^{D}\right) \circ \mathcal{A}_{i}^{D^{ \pm}} \circ\left(S^{D}\right)^{-1}$. 
A brief computation yields the following forward left-invariant differences

$$
\begin{aligned}
\left(\tilde{\mathcal{A}}_{1}^{D^{+}} G_{\boldsymbol{\psi}}^{D} \mathbf{f}\right)[l, m] & =\frac{e^{\frac{-2 \pi i L m}{M}}\left(G^{D} \boldsymbol{\psi}^{\mathbf{f})[l+1, m]-G^{D}} \boldsymbol{\psi}^{\mathbf{f}[l, m]}\right.}{K^{-1}} \\
\left(\tilde{\mathcal{A}}_{2}^{D^{+}} G_{\boldsymbol{\psi}}^{D} \mathbf{f}\right)[l, m] & =M N^{-1}\left(G_{\boldsymbol{\psi}}^{D} \mathbf{f}[l, m+1]-G_{\boldsymbol{\psi}}^{D} \mathbf{f}[l, m]\right) \\
\left(\tilde{\mathcal{A}}_{3}^{D^{+}} G_{\boldsymbol{\psi}}^{D} \mathbf{f}\right)[l, m] & =Q\left(e^{\frac{-2 \pi i}{Q}}-1\right) G_{\boldsymbol{\psi}}^{D} \mathbf{f}[l, m]
\end{aligned}
$$

and the following backward left-invariant differences:

$$
\begin{aligned}
& \left(\tilde{\mathcal{A}}_{1}^{D^{-}} G_{\boldsymbol{\psi}}^{D} \mathbf{f}\right)[l, m]=\frac{\left(G^{D} \mathbf{\psi}\right)[l, m]-e^{\frac{2 \pi i L m}{M}} G^{D} \boldsymbol{\psi}^{\mathbf{f}[l-1, m]}}{K^{-1}} \\
& \left(\tilde{\mathcal{A}}_{2}^{D^{-}} G_{\boldsymbol{\psi}}^{D} \mathbf{f}\right)[l, m]=M N^{-1}\left(G_{\boldsymbol{\psi}}^{D} \mathbf{f}[l, m]-G_{\boldsymbol{\psi}^{D}}^{D} \mathbf{f}[l, m-1]\right) \\
& \left(\tilde{\mathcal{A}}_{3}^{D^{-}} G_{\boldsymbol{\psi}}^{D} \mathbf{f}\right)[l, m]=Q\left(1-e^{\frac{2 \pi i}{Q}}\right) G_{\boldsymbol{\psi}}^{D} \mathbf{f}[l, m] .
\end{aligned}
$$

The discrete operators are exact on the discrete quotient group $\mathfrak{h}_{r}$ and they are first order approximation of the corresponding continuous operators. For example, on the one hand we have for $f$ compactly supported on $[0,1]$ and both $f$ and $\psi$ Riemann-integrable on $\mathbb{R}$ :

$$
\begin{aligned}
\tilde{\mathcal{A}}_{1} \mathcal{G}_{\psi} f\left(p=\frac{l}{K}, q=\frac{m K}{P}\right) & =\left(\partial_{p}-2 \pi q\right)\left(e^{2 \pi i p q} \int_{\mathbb{R}} \overline{\psi(\xi-p)} f(\xi) e^{-2 \pi i \xi q} \mathrm{~d} \xi\right)\left(p=\frac{l}{K}, q=\frac{m K}{P}\right) \\
& =-e^{\frac{2 \pi i l}{P}} \int_{\mathbb{R}} \bar{\psi}^{\prime}\left(\xi-\frac{l}{K}\right) f(\xi) e^{-\frac{2 \pi i n m K}{N P}} \mathrm{~d} \xi \\
& =O\left(\frac{1}{N}\right)-\frac{1}{N} e^{\frac{2 \pi i l m}{P}} \sum_{n=0}^{N-1} \bar{\psi}^{\prime}\left(\frac{n}{N}-\frac{l}{K}\right) f\left(\frac{n}{N}\right) e^{-\frac{2 \pi i n m}{M}} .
\end{aligned}
$$

Whereas on the other hand we have

$$
\left[G_{\boldsymbol{\psi}}^{D} \mathbf{f}\right](l, m)=\frac{1}{N} e^{\frac{2 \pi i m l}{P}} \sum_{n=0}^{N-1} e^{-\frac{2 \pi i n m}{M}} \bar{\psi}\left(\frac{n}{N}-\frac{l}{K}\right) f\left(\frac{n}{N}\right)
$$

so that straightforward computation yields

$$
\begin{aligned}
\tilde{\mathcal{A}}_{1}^{D^{+}} G_{\boldsymbol{\psi}}^{D} \mathbf{f}[l, m] & =\frac{1}{N} e^{2 \pi i \frac{l m}{P}} \sum_{n=0}^{N-1} \frac{\bar{\psi}\left(\frac{n}{N}-\frac{l+1}{K}\right)-\bar{\psi}\left(\frac{n}{N}-\frac{l}{K}\right)}{K^{-1}} f\left(\frac{n}{N}\right) e^{-\frac{2 \pi i n m}{N}} \\
& =O\left(\frac{1}{K}\right) O\left(\frac{1}{N}\right)-\frac{1}{N} e^{\frac{2 \pi i l m}{P}} \sum_{n=0}^{N-1} \bar{\psi}^{\prime}\left(\frac{n}{N}-\frac{l}{K}\right) f\left(\frac{n}{N}\right) e^{-\frac{2 \pi i n m}{M}}
\end{aligned}
$$

So from (4.47) and (4.48) we deduce that

$$
\tilde{\mathcal{A}}_{1}^{D^{+}} G_{\boldsymbol{\psi}}^{D} \mathbf{f}[l, m]=O\left(\frac{1}{N}\right)+\tilde{\mathcal{A}}_{1} \mathcal{G}_{\psi} f\left(p=\frac{l}{K}, q=\frac{m K}{P}\right) .
$$

So clearly the discrete left-invariant vector fields acting on the discrete Gabor-transforms converge to the continuous vector fields acting on the continuous Gabor transforms pointwise as $N \rightarrow \infty$.

However, if it comes to algorithms it is essential that one works on the finite group with corresponding left-invariant vector fields. This is simply due to the fact that one implements finite Gabor-transforms to avoid sampling errors on the grid. The domain of these finite Gabor transforms (4.41) is the group $\mathfrak{h}_{r}$. So, rather than considering the discrete left-invariant vector fields $\tilde{\mathcal{A}}_{i}^{D^{+}}, i=1,2,3$, acting on $G_{\boldsymbol{\psi}}^{D} \mathbf{f}$ as an approximation of the continuous left-invariant vector fields $\tilde{\mathcal{A}}_{i}, i=1,2,3$ acting on $\mathcal{G}_{\psi} f$, one should consider the continuous left-invariant vector fields $\tilde{\mathcal{A}}_{i}$ acting on $\mathcal{G}_{\psi} f$ as an approximation of the discrete left-invariant vector fields $\tilde{\mathcal{A}}_{i}^{D^{+}}, i=1,2,3$ acting on $G_{\boldsymbol{\psi}}^{D}$ f. 
Remark 4.7. In the PDE-schemes which we will present in the next sections, such as for example the diffusion scheme in Section 7, the solutions will leave the space of Gabor-transforms. In such cases one has to apply a left-invariant finite difference to a smooth function $\Phi \in \mathbb{L}_{2}\left(H_{r}\right)$ defined on the Heisenberg-group $H_{r}$ or one has to apply a finite difference to a smooth function $\tilde{\Phi} \in \mathbb{L}_{2}\left(\mathbb{R}^{2}\right)$ defined on phase space, which is not the Gabor-transform of some image. In such cases it is usually not appropriate to use the final results in (4.44) and (4.43) on the group $H_{r}$. In stead one should just use

$$
\begin{aligned}
\left(\mathcal{A}_{1}^{D^{+}} \Phi\right)[l, m, k] & =\left(\mathrm{d} \mathcal{R}^{D^{+}}[1,0,0] \Phi\right)[l, m, k]=\frac{\Phi[[l, m, k][1,0,0]]-\Phi[l, m, k]}{K-1} \\
\left(\mathcal{A}_{1}^{D^{-}} \Phi\right)[l, m, k] & =\left(\mathrm{d} \mathcal{R}^{D^{-}}[1,0,0] \Phi\right)[l, m, k]=\frac{\Phi[l, m, k]-\Phi[l, m, k][-1,0,0]]}{K-1} \\
\left(\mathcal{A}_{2}^{D^{+}} \Phi\right)[l, m, k] & =\left(\mathrm{d} \mathcal{R}^{D^{+}}[0,1,0] \Phi\right)[l, m, k]=\frac{\Phi[l, m, k][0,1,0]]-\Phi[l, m, k]}{\left.\left.N^{-1}-1, k\right][0,-1,0]\right]} \\
\left(\mathcal{A}_{2}^{D^{-}} \Phi\right)[l, m, k] & =\left(\mathrm{d} \mathcal{R}^{D^{-}}[0,1,0] \Phi\right)[l, m, k]=\frac{\Phi[l, m, k]-\Phi[l, m, k, 1}{N^{-1}}
\end{aligned}
$$

which does not require any interpolation between the discrete data iff $\frac{Q}{2 P} \in \mathbb{N}$. However, the left-invariant operators on phase space (4.45) and (4.46) are naturally extendable to $\mathbb{L}_{2}\left(\mathbb{R}^{2}\right)$. For example, $\left.\mathcal{A}_{1}^{D^{+}} \Phi\right)[l, m]=\left[\mathcal{S}^{D} \circ \mathcal{A}_{1}^{D^{+}} \circ\left(\mathcal{S}^{D}\right)^{-1} \tilde{\Phi}\right][l, m]=K\left(e^{-\frac{2 \pi i m}{P}} \tilde{\Phi}[l+1, m]-\tilde{\Phi}[l, m]\right)$ for all $\tilde{\Phi} \in \ell_{2}(\{0, \ldots, K-1\} \times\{0, \ldots, M-1\})$.

\subsection{The Cauchy-Riemann relations in the discrete case}

In contrast to the continuous setting, recall (2.27) and (2.31), the discrete setting the CauchyRiemann relations are not exactly satisfied if one uses the sampled Gaussian kernel (3.36). Nevertheless, it is interesting to find the discrete kernel $\psi_{a}^{D}:=\{\psi[n]\}_{n=-(N-1)}^{N-1}$ such that the following discrete Cauchy-Riemann equation in discrete phase space (using central left-invariant differences) holds

$$
\forall_{l=0, \ldots, K-1} \forall_{m=0, \ldots, M-1} \forall_{\mathbf{f} \in \ell_{2}(I)}: \frac{1}{a}\left(\mathcal{A}_{2}^{D^{+}}+\mathcal{A}_{2}^{D^{-}}\right)+i a\left(\mathcal{A}_{1}^{D^{+}}+\mathcal{A}_{1}^{D^{-}}\right)\left(G_{\boldsymbol{\psi}_{a}^{D}}^{D} \mathbf{f}\right)[l, m]=0,
$$

which is (by left-invariance of the discrete left-invariant vector fields) equivalent to

$$
\begin{aligned}
& \forall l=0, \ldots, K-1 \forall_{m=0, \ldots, M-1} \forall_{n^{\prime}=0, \ldots, L-1}: \frac{1}{a}\left(\mathcal{A}_{2}^{D^{+}}+\mathcal{A}_{2}^{D^{-}}\right)+i a\left(\mathcal{A}_{1}^{D^{+}}+\mathcal{A}_{1}^{D^{-}}\right)\left(G_{\boldsymbol{\psi}_{a}^{D}}^{D} \delta_{n^{\prime}}\right)[l, m]=0, \\
& \text { with } \delta_{n^{\prime}}[n]=\delta_{n n^{\prime}},
\end{aligned}
$$

Now $G_{\boldsymbol{\psi}} \delta_{n^{\prime}}[l, m]=\frac{1}{N} \psi\left[n^{\prime}-l L\right] e^{2 \pi i\left(l m \frac{L}{M}-n^{\prime}\right)}$ and by straightforward application of the discrete left-invariant vector fields we find

$$
K \frac{i a}{2} \frac{e^{\frac{2 \pi i l m L}{M}}}{N}\left(\psi\left[n^{\prime}-(l+1) L\right]-\psi\left[n^{\prime}-(l-1) L\right]\right)+\frac{e^{\frac{2 \pi i l m L}{M}}}{a} \frac{M}{2 N^{2}}\left(\psi\left[n^{\prime}-l L\right]\right)\left(e^{2 \pi i \frac{l L}{M}}+e^{-2 \pi i \frac{l L}{M}}\right)=0 .
$$

Consequently, we get the following 2-fold recursions (enumerated by $l$ making steps of $L$ in $\{0, \ldots, N-1\})$ for the even discrete kernel $\boldsymbol{\psi}_{a}^{D}:=\{\psi[n]\}_{n=-(N-1)}^{N-1}=\left\{\psi\left[n^{\prime}-l L\right]\right\}_{n^{\prime}=0, l=0}^{L-1, K-1}$ :

$$
\left\{\begin{array}{l}
\frac{N K}{2 M} a^{2}\left(\psi\left[n^{\prime}-(l+1) L\right]-\psi\left[n^{\prime}-(l-1) L\right]\right)+\psi\left[n^{\prime}-l L\right] \sin \left(\frac{2 \pi l L}{M}\right), \text { for } l=0, \ldots K-1, \\
\psi[-n]=\psi[n], \psi[N]:=\psi[0], \psi[-N]:=\psi[0] \text { and } \sum_{l=0}^{K-1} \psi\left[n^{\prime}-l L\right]=1
\end{array}\right.
$$

In particular if we consider the safe case of extreme oversampling $K=M=N, L=1, P=N$ we get a unique solution. Figure 3 shows a comparison of this discrete solution and the solution of the continuous Cauchy-Riemann equations (2.31), using the fundamental transformation $p=\frac{l}{N}$, $q=\frac{m M}{N}$ (needed to let the discrete Gabor transform converge to the continuous Gabor transform, recall (3.39)) for several scaling values of $a>0$. We conclude that the important Cauchy-Riemann relations which we shall exploit in the next section also hold in the discrete setting. The CauchyRiemann equations require a slightly different kernel than the discretely sampled kernel required in the continuous setting, but for reasonable parameter settings these kernels are rather close (and in the limiting case $N \rightarrow \infty$ they are the same). Consequently, the results of the next section, where we relate our algorithms based on the theoretical approach by Daudet et al. [9] to simple erosion schemes [5], apply to the discrete as well. 

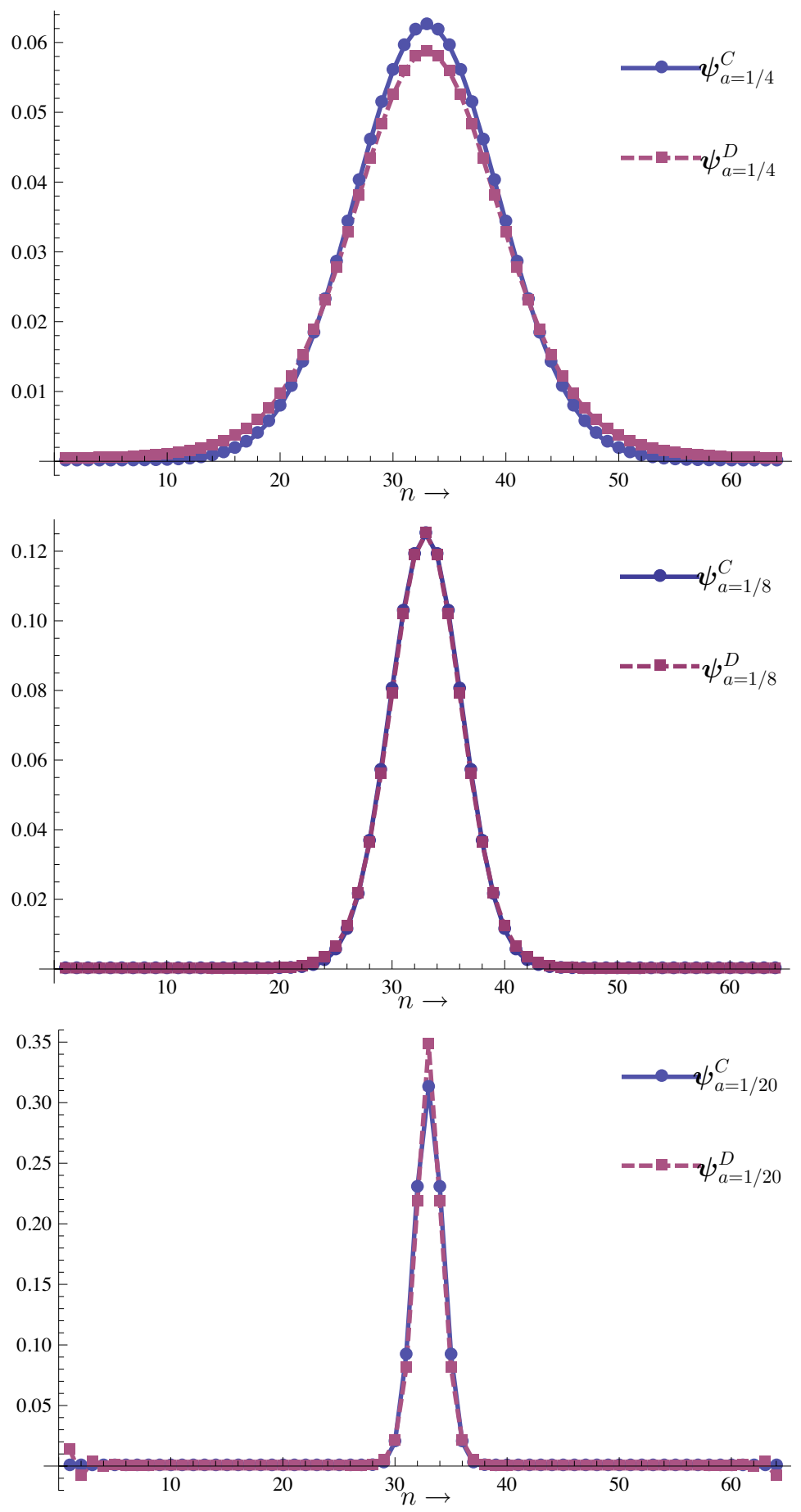

Figure 3: A comparison between the solution $\boldsymbol{\psi}_{a}^{D}=\{\psi[n]\}_{n=-(N-1)}^{N-1}$ of the discrete Cauchy Riemann equations (4.50), for the case $K=M=N, L=1, P=N$ and the discrete sampling $\boldsymbol{\psi}_{a}^{C}=\left\{\psi_{a}\left(\frac{n}{N}\right)\right\}_{n=-(N-1)}^{N-1}$ of the continuous solution $\psi_{a}(\xi)$ (2.30), or rather (3.38) where the reflection to the negative index-set $\{-(N-1), \ldots,-1\}$ is applied only to make the convolution in the definition of the discrete Gabor transform (3.34) periodic and well-defined, of (2.31), for different values of $a>0$. Note that we fixed the number of samples $N=64$. As $a>0$ decreases the solution of the discrete Cauchy Riemann solutions converges pointwise to (the sampled) continuous solution (although if $a$ becomes too small the distribution is to peaked for decent sampling). Similar behavior is obtained if we keep $a>0$ fixed and increase the number of samples $N$. This coincides with our fundamental convergence results (3.39) and (4.48). 


\section{Phase invariant convection (Reassignment) on Gabor trans- forms}

First we derive left-invariant and phase-invariant differential operators on Gabor transforms $U:=$ $\mathcal{W}_{\psi}(f)$, which will serve as generators of left-invariant phase-invariant convection equations (i.e. reassignment) on Gabor transforms. The basic goal of reassignment is to sharpen the Gabor distributions towards lines (which are close to the geodesics derived in section D.2 of Appendix D.2) in $H_{r}$, while maintaining the signal as much as possible.

On the group $H_{r}$ it directly follows by the product rule for differentiation that the following differential operators $\mathcal{C}: \mathcal{H}_{n} \rightarrow \mathcal{H}_{n}$ given by

$$
\mathcal{C}(U)=M(|U|)\left(-\mathcal{A}_{2} \Omega \mathcal{A}_{1} U+\mathcal{A}_{1} \Omega \mathcal{A}_{2} U\right), \quad \text { where } \Omega=\arg \{U\},
$$

are phase invariant, where $M(|U|)$ denotes a multiplication operator with the modulus of $U$ naturally associated to a bounded monotonically increasing differentiable function $M:[0, \max (U)] \rightarrow$ $[0, M(\max (U))] \subset \mathbb{R}$ with $M(0)=0$. Here we note that the Gabor modulus transform is bounded, since the space of Gabor transforms is a reproducing kernel ${ }^{5}$ space $\mathbb{C}_{K}^{H_{r}}$ consisting of functions on $H_{r}$ with a uniform bound on point evaluation and the window $\psi$ is assumed to be within the Fourier invariant Schwarz space of rapidly decaying functions. Furthermore, these considerations show us that $\mathcal{C}$ can be considered as an unbounded operator from $\mathcal{H}_{n}$ into $\mathcal{H}_{n}$, since the space $\mathcal{H}_{n}$ is right-invariant and invariant under bounded multiplication operators which do not depend on $z=e^{2 \pi i s}$, i.e.

$$
\begin{aligned}
& U=\mathcal{W}_{\psi}(f) \in \mathcal{H}_{n} \Rightarrow \partial_{t}-\mathcal{A}_{i} U \in \mathcal{H}_{n}, \\
& U \in \mathcal{H}_{n} \Rightarrow M(|U|) U \in \mathcal{H}_{n}, \\
& U \in \mathcal{H}_{n} \Rightarrow \mathcal{C}(U) \in \mathcal{H}_{n} .
\end{aligned}
$$

Now a direct calculation using the product rule for differentiation yields:

$$
\mathcal{C}\left(e^{i \Omega}|U|\right)=M(|U|) e^{i \Omega}\left(-\mathcal{A}_{2} \Omega \mathcal{A}_{1}|U|+\mathcal{A}_{1} \Omega \mathcal{A}_{2}|U|\right)
$$

and for Gaussian kernels $\psi_{a}(\xi)=e^{-a^{-2} \xi^{2} n \pi}$ we may apply the Cauchy Riemann relations $(2.26)$ which simplifies for the special case $M(|U|)=|U|$ to

$$
\mathcal{C}\left(e^{i \Omega}|U|\right)=|U| e^{i \Omega}\left(-\mathcal{A}_{2} \Omega \mathcal{A}_{1}|U|+\mathcal{A}_{1} \Omega \mathcal{A}_{2}|U|\right)=\left(a^{2}\left(\partial_{p}|U|\right)^{2}+a^{-2}\left(\partial_{q}|U|\right)^{2}\right) e^{i \Omega} .
$$

Now consider the following phase-invariant adaptive convection equation on $H_{r}$,

$$
\left\{\begin{array}{l}
\partial_{t} W(g, t)=-\mathcal{C}(W(\cdot, t))(g) \\
W(g, 0)=U(g)
\end{array}\right.
$$

with either

$$
\begin{aligned}
& \text { 1. } \mathcal{C}(W(\cdot, t))=M(|U|)\left(-\mathcal{A}_{2} \Omega, \mathcal{A}_{1} \Omega\right) \cdot\left(\mathcal{A}_{1} W(\cdot, t), \mathcal{A}_{2} W(\cdot, t)\right) \text { or } \\
& \text { 2. } \mathcal{C}(W(\cdot, t))=e^{i \Omega}\left(a^{2} \frac{\left(\partial_{p}|W(\cdot, t)|\right)^{2}}{|W(\cdot, t)|}+a^{-2} \frac{\left(\partial_{q}|W(\cdot, t)|\right)^{2}}{|W(\cdot, t)|}\right)
\end{aligned}
$$

In the first choice we stress that $\arg (W(\cdot, t))=\arg (W(\cdot, 0))=\Omega$, since transport only takes place along iso-phase surfaces. Initially, in case $M(|U|)=1$ the two approaches are the same since at $t=0$ the Cauchy Riemann relations (2.32) hold, but after a while the Cauchy-Riemann equations are violated over time (this directly follows by the preservation of phase and the non-preservation of amplitude in both approaches), which has been more or less overlooked in the convection schemes in $[9,7]$. Nevertheless, both approaches (5.53) in (5.52) make sense from a practical point of view.

\footnotetext{
${ }^{5}$ The reproducing kernel $K$ of the space of Gabor transforms relates to the window in the following manner: $K(g, h)=\left(\mathcal{U}_{g} \psi, \mathcal{U}_{h} \psi\right)$.
} 
For small convection times $t>0$ the difference turns out to be hardly visible, but after a while we have observed differences. See for example Figure 8 (where the green and yellow signal correspond to the first choice whereas the magenta signal corresponds to the second choice).

The second choice in (5.53) in (5.52) is just a phase-invariant inverse Hamilton Jakobi equation on $H_{r}$, with a Gabor transform as initial solution. Rather than computing the viscosity solution of this non-linear PDE, we may as well store the phase and apply an inverse Hamilton Jakobi system on $\mathbb{R}^{2}$ with the amplitude $|U|$ as initial condition and multiply with the stored phase factor afterwards. Here we stress that by taking the modulus, we omit the $s$-dependence and we automatically apply our algorithm in phase space.

With respect to the first choice in (5.53) in (5.52), which is much more cumbersome to implement, the authors in [9] considered the equivalent equation on phase space:

$$
\left\{\begin{array}{l}
\partial_{t} \tilde{W}(p, q, t)=-\tilde{\mathcal{C}}(\tilde{W}(\cdot, t))(p, q) \\
\tilde{W}(p, q, 0)=\tilde{U}(p, q)=e^{i \tilde{\Omega}}|\tilde{U}|=e^{i \tilde{\Omega}}|U|
\end{array}\right.
$$

where $\tilde{U}(p, q)=\mathcal{G}_{\psi} f(p, q)$ and

$$
\tilde{C}(\tilde{W}(\cdot, t))=M(|U|)\left(-\tilde{\mathcal{A}}_{2} \tilde{\Omega} \mathcal{A}_{1} \tilde{W}(\cdot, t)+\tilde{\mathcal{B}}_{1} \tilde{\Omega} \mathcal{A}_{2} \tilde{W}(\cdot, t)\right)
$$

where we recall $(2.29)$ and $\mathcal{G}_{\psi}(f)=\mathcal{S} \mathcal{W}_{\psi}(f)$ and $\mathcal{A}_{i}=\mathcal{S}^{-1} \tilde{\mathcal{A}}_{i} \mathcal{S}$ for $i=1,2,3$. Note that the authors in [9] consider the case $M=1$. However the case $M=1$ and the earlier mentioned case $M(|U|)=|U|$ are equivalent by transformations $|U| \rightarrow \log |U|$ and $|U| \rightarrow \exp |U|$, since

$$
\frac{\partial}{\partial t}|U|=a^{2} \frac{\left(\partial_{p}|U|\right)^{2}}{|U|}+a^{-2} \frac{\left(\partial_{q}|U|\right)^{2}}{|U|} \Leftrightarrow \frac{\partial}{\partial t} \log |U|=a^{2}\left(\partial_{p} \log |U|\right)^{2}+a^{-2}\left(\partial_{q} \log |U|\right)^{2} .
$$

Although the approach by the authors in [9] is highly plausible from the theoretical point of view, the authors did not provide an explicit computation scheme and here our technical results of the previous section come at hand.

On the other hand with the second approach in (5.53) one does not need the technicalities of the previous section, since here the viscosity solution of the system (5.54) is given by a basic inverse convolution over the $(\max ,+)$ algebra, [5], (also known as erosion operator in image analysis)

$$
\tilde{W}(p, q, t)=\left(K_{t} \ominus|U|\right)(p, q) e^{i \Omega(p, q, t)},
$$

with the kernel

$$
K_{t}(p, q)=-\frac{a^{-2} p^{2}+a^{2} q^{2}}{4 t}
$$

(describing the growth of balls in $\mathbb{R}^{2}$ ), where

$$
(f \ominus g)(p, q)=\inf _{\left(p^{\prime}, q^{\prime}\right) \in \mathbb{R}^{2}}\left[g\left(p^{\prime}, q^{\prime}\right)-f\left(p^{\prime}-p, q^{\prime}-q\right)\right] .
$$

Here we note that the homomorphism between dilation/erosion and diffusion/inverse diffusion is given by the Cramer transform $C=\mathfrak{F} \circ \log \circ \mathcal{L}$, [5], [1], which is a concatination of the multivariate Laplace transform, logarithm and Fenchel transform. The Fenchel transform maps a convex function $c: \mathbb{R}^{d} \rightarrow \overline{\mathbb{R}}$ (in particular $d=2$ ) onto the function $\mathbf{x} \mapsto[\mathfrak{F} c](\mathbf{x})=\sup _{\mathbf{y} \in \mathbb{R}^{n}}[\mathbf{y} \cdot \mathbf{x}-c(\mathbf{x})]$. The isomorphic property of the Cramer transform is summarized as

$$
\mathcal{C}(f * g)=\mathfrak{F} \log \mathcal{L}(f * g)=\mathfrak{F}(\log \mathcal{L} f+\mathcal{L} g)=\mathfrak{F} \log \mathcal{L} f \oplus \mathfrak{F} \log \mathcal{L} g=\mathcal{C} f \oplus \mathcal{C} g,
$$

where the convolution on the (max, + )-algebra is given by $f \oplus g(\mathbf{x})=\sup _{\mathbf{y} \in \mathbb{R}^{d}}[f(\mathbf{x}-\mathbf{y})+g(\mathbf{y})]$.

Remark 5.8. Note that the effective operator on the modulus $|U| \mapsto a^{2}\left(\partial_{p}|U|\right)^{2}+a^{-2}\left(\partial_{q}|U|\right.$, that arises from (5.51), is not left-invariant. Nevertheless the net-operator $\mathcal{C}\left(e^{i \Omega}|U|\right)=a^{2}\left(\partial_{p}|U|\right)^{2}+$ $a^{-2}\left(\partial_{q}|U|\right)^{2} e^{i \Omega}$ is left-invariant. In general it is wrong to apply a left-invariant operator to the modulus and to restore the old phase afterwards, since this violates left-invariance. This directly follows by the fact that $\arg \circ \mathcal{L}_{g} \circ \mathcal{W}_{\psi} f \neq \mathcal{L}_{g} \circ \arg \mathcal{W}_{\psi} f$. 


\subsection{Algorithm for the first choice in the discrete setting}

Here we provide an explicit algorithm on the discrete Gabor transform $G_{\boldsymbol{\psi}}^{D} \mathbf{f}$ of the discrete signal $\mathbf{f}$, that corresponds to the theoretical PDE's on the continuous case as proposed in [9], i.e. convection equation (5.52) where we apply the first choice (5.53). Recall that the domain of this transform is the phase space associated discrete group $\mathfrak{h}_{r}$. Although that the PDE by [9] is not as simple as the second approach in (5.53) (which corresponds to a standard erosion step on the absolute value $\left|\mathcal{G}_{\psi} f\right|$ followed by a restoration of the phase afterwards) we do provide an explicit numerical scheme of this PDE, where we will stay entirely in the phase space of the discrete setting.

Explicit upwind scheme with left-invariant finite differences in pseudo-code:

For $l=1, \ldots, K-1, m=1, \ldots M-1$ set $W[l, m, 0]:=\mathcal{G}_{\psi} f[l, m]$.

For $t=1, \ldots, T$

For $l=0, \ldots, K-1$, for $m=1, \ldots, M-1 \operatorname{set}^{6}$

$\tilde{v}^{1}[l, m, t]:=-\frac{a K}{2}(|W[l+1, m, t=0]|-|W[l-1, m, t=0]|) \cdot \frac{\left(M\left(\left|\mathcal{G}_{\psi} f\right|\right)\right)[l, m]}{\left|\mathcal{G}_{\psi} f\right|[l, m]}$

$\tilde{v}^{2}[l, m, t]:=-\frac{a M}{2}(|W[l, m+1, t=0]|-|W[l, m-1, t=0]|) \cdot \frac{\left(M\left(\left|\mathcal{G}_{\psi} f\right|\right)\right)[l, m]}{\left|\mathcal{G}_{\psi} f\right|[l, m]}$

$W[l, m, t]:=W[l, m, t-1]+K \Delta t\left(z^{+}\left(\tilde{v}^{1}\right)[l, m, t]\left[\mathcal{A}_{1}^{D^{-}} W\right][l, m, t]+z^{-}\left(\tilde{v}^{1}\right)[l, m, t]\left[\mathcal{A}_{1}^{D^{+}} W\right][l, m, t]\right)+$

$M \Delta t\left(z^{+}\left(\tilde{v}^{2}\right)[l, m, t]\left[\mathcal{A}_{2}^{D^{-}} W\right][l, m, t]+z^{-}\left(\tilde{v}^{2}\right)[l, m, t]\left[\mathcal{A}_{2}^{D^{+}} W\right][l, m, t]\right) . \quad$ with $z^{+}(\phi)[l, m, t]=\max \{\phi(l, m, t), 0\}$

and $z^{-}(\phi)[l, m, t]=\min \{\phi(l, m, t), 0\}$.

Remark 5.9. The Cauchy-Riemann relations only hold at $t=0$, therefore we set $\tilde{v}^{1}[l, m, t]:=$ $-\frac{a K}{2}(|W(l+1, m, 0)|-|W(l-1, m, 0)|)$. This means that the velocity vector $\tilde{v}=\left(\tilde{v}^{1}, \tilde{v}^{2}\right)$ is in fact not dependent on time. It is not adapted to the evolving Gabor transform, but rather on the initial Gabor transform. This is different than our second approach (5.53) in the convection PDE (5.52), where the velocity is adaptive to the evolving Gabor transform. A third approach arises if one replaces $\tilde{v}^{1}[l, m, t]:=-\frac{a K}{2}(|W(l+1, m, 0)|-|W(l-1, m, 0)|)$ by $\tilde{v}^{1}[l, m, t]:=-\frac{a K}{2}(|W(l+1, m, t)|-|W(l-1, m, t)|)$ and $\tilde{v}^{2}[l, m, t]:=-\frac{a M}{2}(|W(l, m+1,0)|-|W(l, m-1,0)|)$ by $\tilde{v}^{2}[l, m, t]:=-\frac{a M}{2}(|W(l, m+1, t)|-|W(l, m-1, t)|)$ in the PDE-scheme above. This adaptive version of the first approach in (5.53) works fine, cf. Figure 8 , and is more adaptive, but it is not entirely phase invariant, since the Cauchy-Riemann relations are more and more violated as $t$ increases.

\subsection{Evaluation Reassignment}

We distinguished between two approaches to apply left-invariant (with respect to the discrete group $\mathfrak{h}_{r}$ with product (4.42)) adaptive convection on discrete Gabor-transforms. Either we apply the numerical upwind PDE-scheme described in subsection 5.1 using the discrete left-invariant vector fields (4.45), (4.46), or we apply erosion (5.55) on the modulus and restore the phase afterwards. These two approaches corresponds to respectively the first and second choice in (5.53). To reduce dimensionality from 3 to 2 all implementations take place in the discrete phase space.

Within each of the two approaches, we distinguish between two cases. Either we can use the discrete Cauchy-Riemann kernel $\boldsymbol{\psi}_{a}^{D}$ or the sampled continuous Cauchy-Riemann kernel $\boldsymbol{\psi}_{a}^{C}$, recall Figure 3. This brings the total amount of methods to four.

To evaluate these 4 proposed methods we apply the reassignment scheme to the reassignment of a linear chirp that is multiplied by a modulated Gaussian and is sampled using $N=128$ samples. The input signal is an analytic signal so it suffices to show its Gabor transform from 0 to $\pi$. A visualization of this complex valued signal can be found in Figure 5 as the topmost image. The other signals in this figure are the reconstructions from the reassigned Gabor transforms that are given in Figure 7. Here the topmost image shows the Gabor transform of the original signal. Apart

\footnotetext{
${ }^{6}$ For $M=1$ it is numerically better to first compute $\log \left|\mathcal{G}_{\boldsymbol{\psi}} \mathbf{f}\right|$ and then apply the discrete vector fields, this avoids divisions by small $\left|\mathcal{G}_{\boldsymbol{\psi}} \mathbf{f}\right|[l, m]$. Note that the chain-rule for differentiation does not apply for discrete vector fields. Setting $M(U)=|U|$ avoids these simple numerical problems.
} 


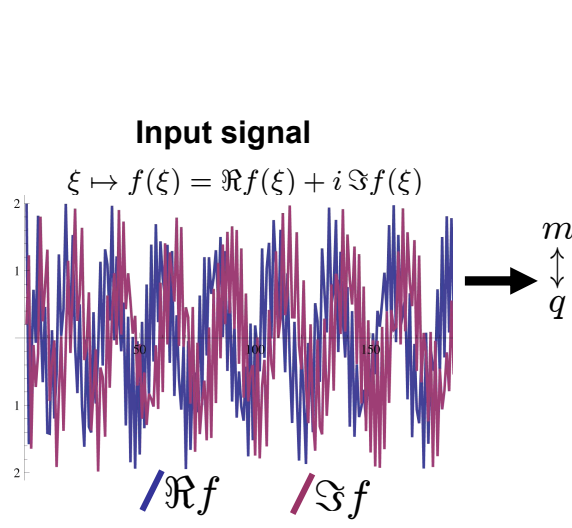

\section{Absolute value of Gabor transform}

\section{Absolute value of re-assigned} Gabor transform

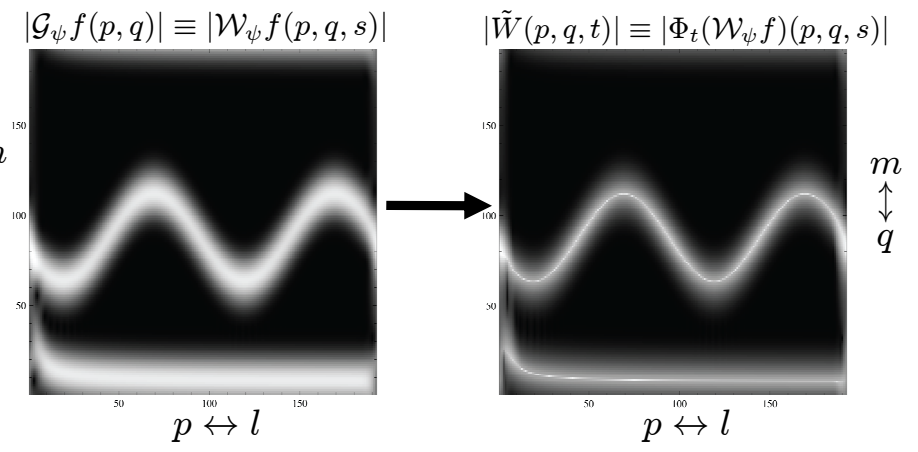

Figure 4: Illustration of re-assignment by adaptive phase-invariant convection explained in Section 5, using the upwind scheme of subsection 5.1 applied on a Gabor transform constructed by Gabor kernel (3.36). Left: input signal, middle: absolute value of the corresponding Gabor transform, right: result of re-assignment in the Gabor domain.

from the first images, from top to bottom in Figure 5 and Figure 7 one can find the reconstructions and reassigned Gabor transforms respectively using the four methods of reassignment. The parameters involved in generating these figures are the number of samples used to sample the chirp signal, which equals $N=128$. The number of samples in the spatial direction is $K=128$, and the number of samples in the frequency direction is $M=128$. This implies $L=1$ and $P=128$. Furthermore $a=1 / 6$ and the time step for the PDE based method is set to $\Delta t=10^{-3}$. All images show a snapshot of the reassignment method that was stopped at $t=0.1$. The signals are scaled such that their energy equals the energy of the input signal. This is needed to correct for the numerical diffusion the discretization scheme suffers from. Reassignment should only reassign time frequency particles (or Gabor atoms), therefore we argue it is justified to compare the methods by rescaling the output such that the $\mathbb{L}_{2}$-norm is conserved. Clearly the reassigned signals resemble the input signal quite well. To study the differences the modulus of the input signal is depicted together with the modulus of the reconstructed reassigned signals in Figure 8. The PDE scheme that uses the sampled continuous window shows some defects. In contrast, the PDE scheme that uses $\boldsymbol{\psi}_{a}^{D}$ resembles the modulus of the original signal the most. Numerical results support these observations. Table 5.2 shows the relative errors for all three experiments of the complex valued signal, $\epsilon_{1}$ and the relative error of its modulus $\epsilon_{2}$, i.e.

$$
\epsilon_{1}=\frac{\|\mathbf{f}-\tilde{\mathbf{f}}\|_{\ell_{2}(I)}}{\|\mathbf{f}\|_{\ell_{2}(I)}}, \quad \epsilon_{2}=\frac{\||\mathbf{f}|-|\tilde{\mathbf{f}}|\|_{\ell_{2}(I)}}{\|\mathbf{f}\|_{\ell_{2}(I)}} .
$$

where $\mathbf{f} \in \ell_{2}(I)$ is the original discrete signal and $\tilde{\mathbf{f}}$ the reconstructed signal after normalized re-assignment in the Gabor domain.

Advantages of the erosion scheme (5.55) over the upwind PDE-scheme of section 5.1 are

1. The erosion scheme does not produce numerical approximation-errors in the phase, which is evident since the phase is not used in the computations.

2. The erosion scheme does not involve numerical diffusion as it does not suffer from finite step-sizes.

3. The erosion scheme is much faster from a computational point of view. ${ }^{7}$

The convection time in the erosion scheme is different than the convection time in the upwindscheme, due to violation of the Cauchy-Riemann equations. Typically, to get similar visual sharpening of the re-assigned Gabor transforms, the convection time of the PDE-scheme should be taken

\footnotetext{
${ }^{7}$ For example the erosions (5.55) are separable, since we use a quadratic structure element (5.56) which is a sum of a square in $p$ and a square in $q$, reducing the complexity of the erosion by order $O(K+M)$ in stead of $O(K M)$.
} 


\begin{tabular}{l|c|c|c|} 
& $\epsilon_{1}$ & $\epsilon_{2}$ & $t$ \\
\hline Erosion continuous window & $2.4110^{-2}$ & $8.3810^{-3}$ & 0.1 \\
Erosion discrete window & $8.2510^{-2}$ & $7.8910^{-2}$ & 0.1 \\
PDE continuous window & $2.1610^{-2}$ & $2.2110^{-3}$ & 0.1 \\
PDE discrete window & $1.4710^{-2}$ & $3.3210^{-4}$ & 0.1 \\
PDE discrete window & $2.4310^{-2}$ & $6.4310^{-3}$ & 0.16
\end{tabular}

Table 1: The first column shows $\epsilon_{1}$, the relative error of the complex valued reconstructed signal compared to the input signal. In the second column $\epsilon_{2}$ can be found which represents the relative error of the modulus of the signals. The rows show the methods that were used in the reassignment of the chirp signal. Parameters involved are grid constants $K=M=N=128$, window scale $a=\frac{1}{8}$ and the convection time $t=0.1$. The time step for the forward Euler method is $\Delta t=10^{-3}$ if applicable. PDE stand for the upwind scheme presented in Subsection 5.1 and morphological means the morphological erosion method given by eq. (5.55).

larger than the convection time of the erosion scheme. We think that this is due to numerical blurring in the PDE-scheme. For example $t=1.6$ for the PDE-scheme roughly corresponds to $t=1$ in the sense that the $\mathbb{L}_{2}$-errors nearly coincide, see Table 5.2 , but even here the erosion scheme seems (at least visually) to sharpen more than the PDE-scheme. The method that uses a sampled version of the continuous window shows large errors. We note that in Figure 8 the defects are clearly visible. This shows the importance of the window selection, i.e. in the PDEschemes it is better to use window $\boldsymbol{\psi}_{a}^{D}$ rather than window $\boldsymbol{\psi}_{a}^{C}$ (in the initial condition/Gabor transform). Especially if it comes to reduction of phase-errors. However, Figure 9 and Table 5.2 clearly indicate that in the erosion schemes it is better to choose window $\boldsymbol{\psi}_{a}^{C}$ than $\boldsymbol{\psi}_{a}^{D}$. 

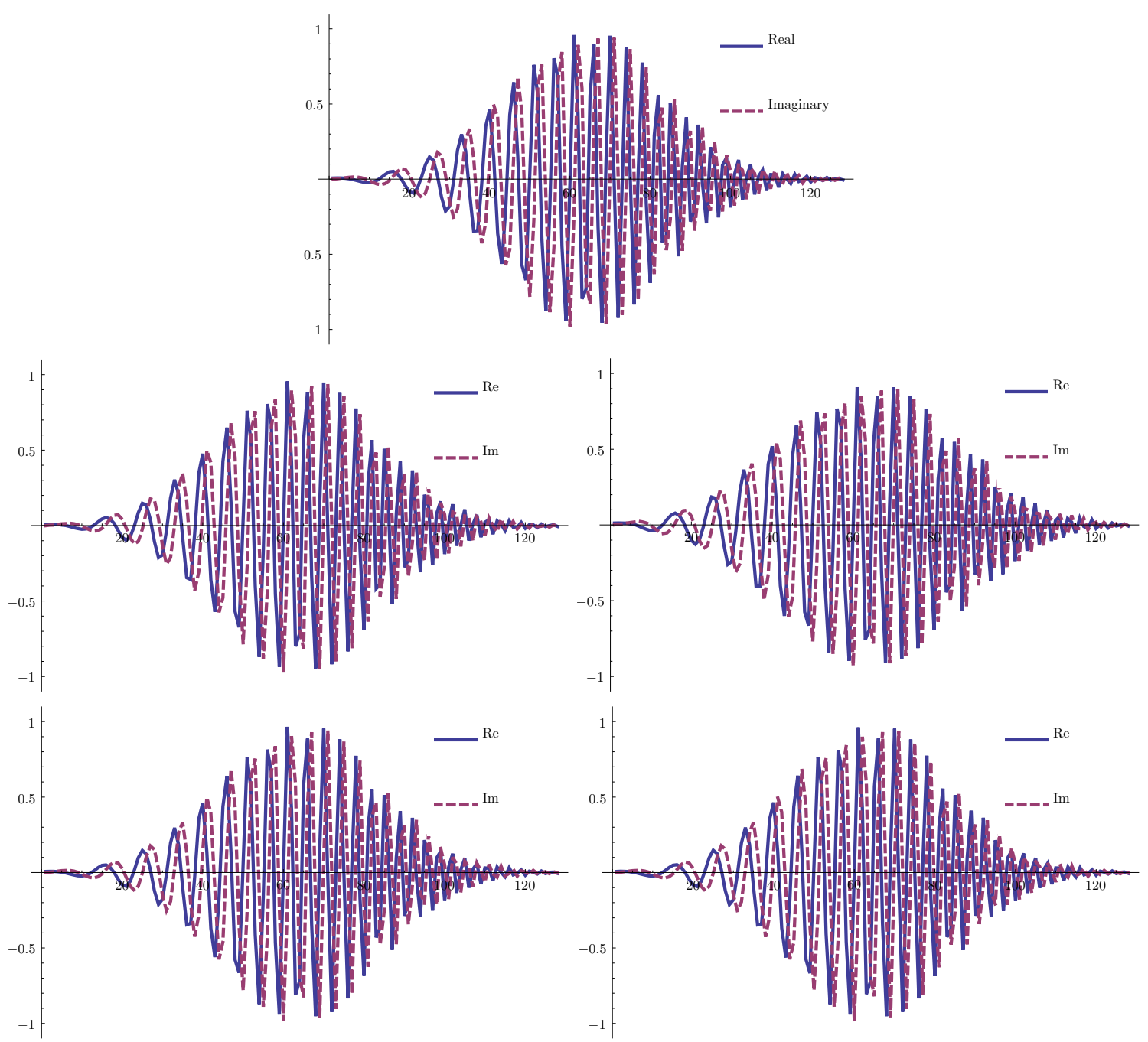

Figure 5: Reconstructions of the reassigned Gabor transforms of the signal that is depicted on top. The signals in the 2nd row correspond to reassignment by means of morphological erosion where in the left-signal we used $\boldsymbol{\psi}_{a}^{C}$ in the Gabor transform and in the right signal we used $\boldsymbol{\psi}_{a}^{D}$. The signals in the 3rd row correspond to reassignment by the upwind scheme (with $M(|U|)=1$ ) of Subsection 5.1, where again in the left-signal we used $\boldsymbol{\psi}_{a}^{C}$ in the Gabor transform and in the right signal we used $\boldsymbol{\psi}_{a}^{D}$. Parameters involved are grid constants $K=M=N=128$, window scale $a=1 / 6$ time step $\Delta t=10^{-3}$ and the convection time $t=0.1$. The goal of reassignment is achieved; all reconstructed signals are close to the original signal, whereas their corresponding Gabor transforms depicted in Figure 6 are much sharper than the Gabor transform of the original. 

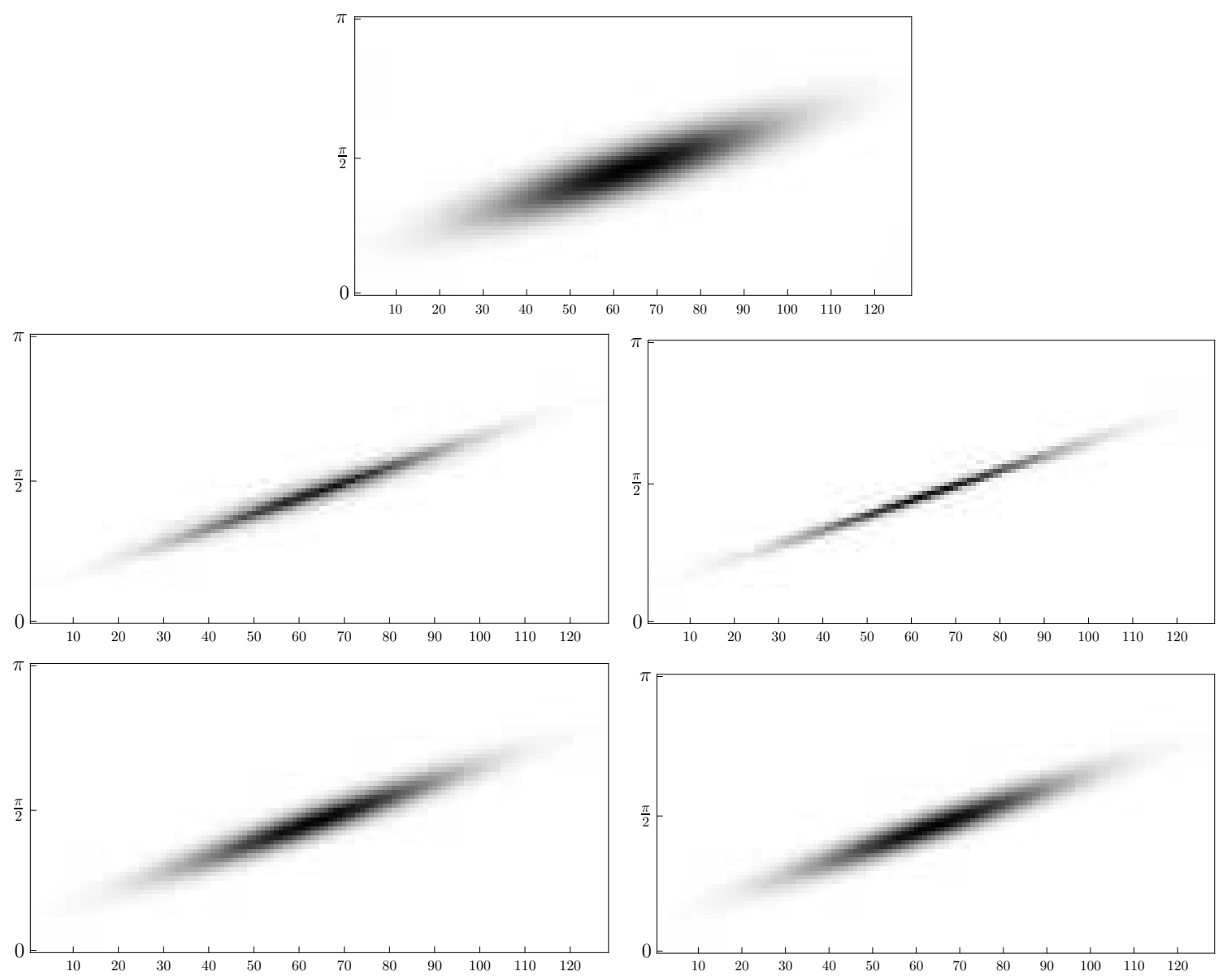

Figure 6: The reassigned Gabon transforms of the signal that is depicted on top. The absolute value of the Gabor transform in the end row correspond to reassignment by means of morphological erosion where in the left Gabon transform we used $\boldsymbol{\psi}_{a}^{C}$ in the Gabon transform and in the Gabon transform depicted on the right we used $\boldsymbol{\psi}_{a}^{D}$. The absolute value of the Gabon transforms in the ard row correspond to reassignment by the upwind scheme (with $M(|U|)=1$ ) of Subsection 5.1, where again in the left Gabon transform we used $\boldsymbol{\psi}_{a}^{C}$ in the Gabon transform and in the Gabon transform depicted on the right we used $\boldsymbol{\psi}_{a}^{D}$. Parameters involved are grid constants $K=M=N=128$, window scale $a=1 / 6$ time step $\Delta t=10^{-3}$ and the convection time $t=0.1$.

26 

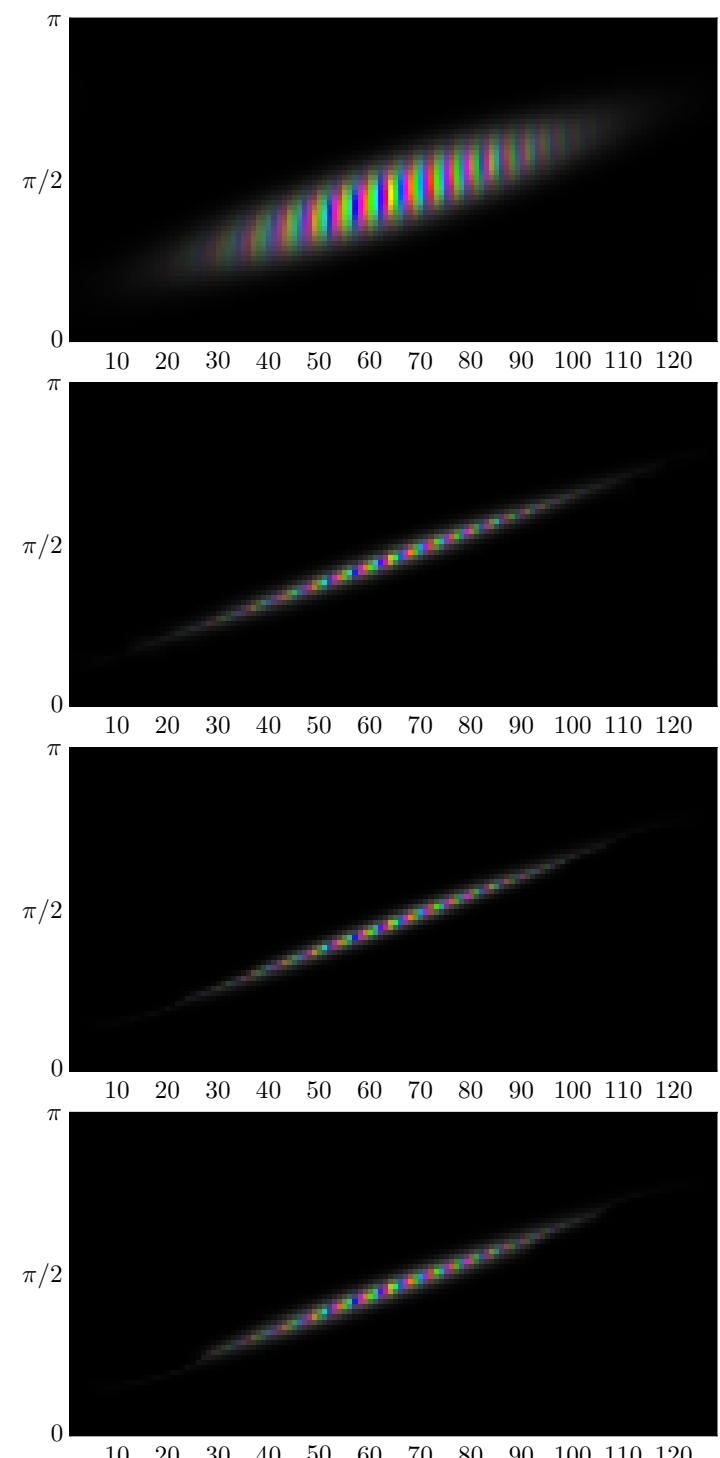

Figure 7: Gabor transforms of the top most signal in Figure 5. The second third and fourth signal correspond to reassignment by means of morphological erosion using $\boldsymbol{\psi}_{a}^{D}$ in the Gabor transform, convection (adaptive according to Remark 5.9, with $M(|U|)=1$ ) using $\boldsymbol{\psi}_{a}^{D}$ in the Gabor transform and convection (adaptive according to Remark 5.9, with $M(|U|)=1$ ) when a sampled version $\boldsymbol{\psi}_{a}^{C}$ of the continuous window $\psi_{a}$ is used respectively. Parameters involved are grid constants $K=M=N=128$, window scale $a=1 / 8$ time step $\Delta t=10^{-3}$ and the convection time $t=0.1$. Note that the adaptation proposed in Remark 5.9 hardly violates the phase-invariance. 


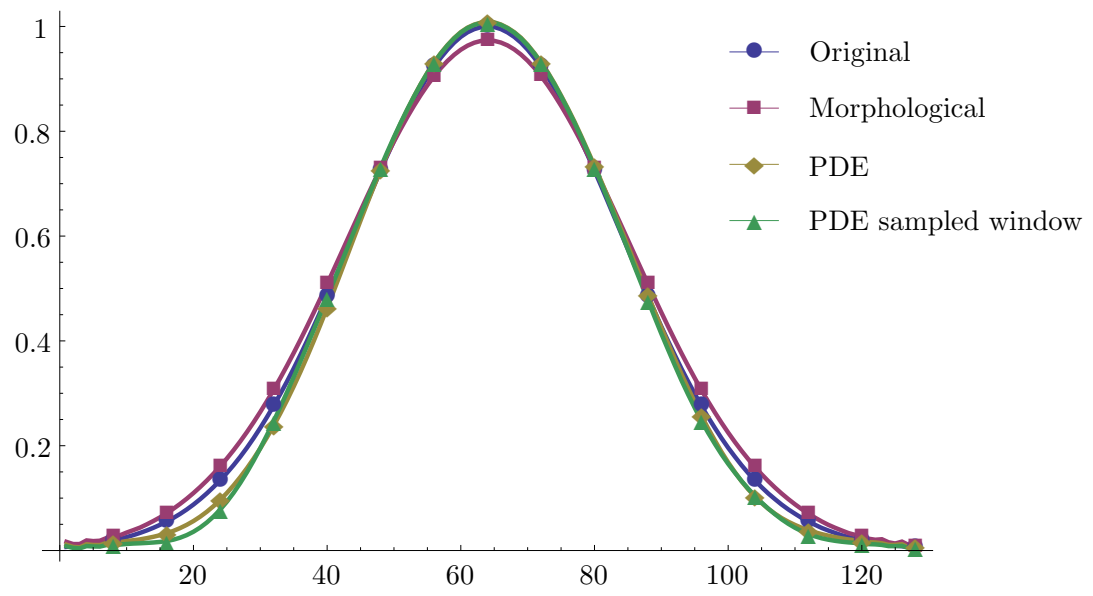

Figure 8: The modulus of the signals corresponding to the Gabor transforms in Figure 7. See the caption of that figure for further details. Note that "PDE-sampled window" stands for the PDE-approach of Subsection 5.1 (with $M=1$ ) applied to discrete Gabor transforms constructed by kernels $\boldsymbol{\psi}_{a}^{C}$, recall Fig.3. The erosion scheme does not perform well in the signal domain if the discrete kernel $\boldsymbol{\psi}_{a}^{D}$ is used. The PDE-scheme performs better if $\boldsymbol{\psi}_{a}^{D}$ is used (rather than $\boldsymbol{\psi}_{a}^{C}$ ).

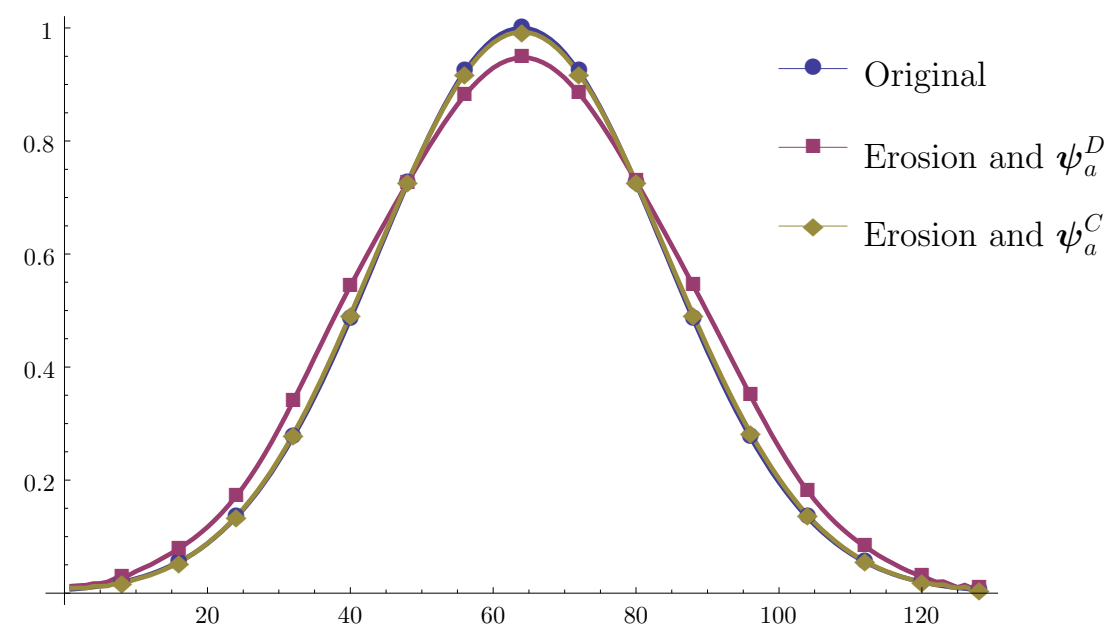

Figure 9: The modulus of the signals corresponding to the Gabor transforms in Figure 6. See caption of Figure 6 for details. Application of erosion (5.55) applied on a Gabor transform constructed with $\boldsymbol{\psi}_{a}^{C}$ performs better than erosion applied on a Gabor transform constructed by $\boldsymbol{\psi}_{a}^{D}$. 

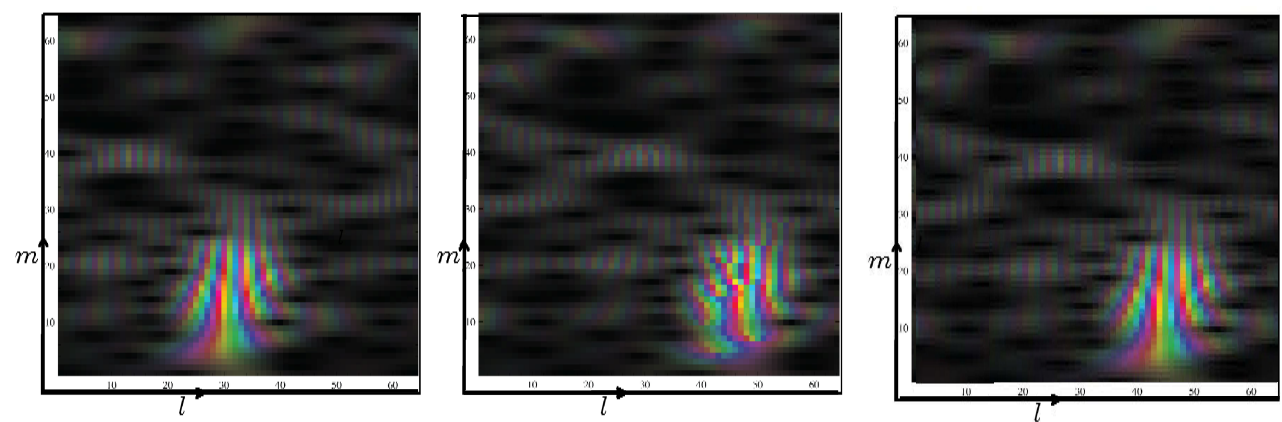

Figure 10: From left to right, the Gabor transform of the original signal depicted in the left of Fig. 2, the result of phase-invariant transport, the result of phase covariant transport (which indeed corresponds to the shift of the original signal). We again used the modulus of the (transformed) Gabor transform for signal strength and applied the same color-coding of the phase as in Fig. 2. Clearly, the phase-invariantly shifted Gabor transform is useless since at strong amplitude locations of the transported Gabor transform, the transported Gabor transform carries the illposed phase of weak amplitude locations in the original Gabor transform. This explains why the corresponding signal of the phase-invariantly shifted Gabor transform is quite distorted, as can be seen in the middle of Fig. 2. Phase invariant operators that are not phase covariant do not make sense.

\section{Phase covariant convection}

In general phase invariant convection does not make sense, as we will show by a very basic experiment in Figure 10. Nevertheless, the special cases of phase-invariant convection considered in the previous section does make sense, since they are also phase covariant, meaning that the phase moves along with the flow. This is a direct consequence of the fact that the flow takes place along isocontours of the phase, in which case it clearly does not matter if one maintains the old phase (phase invariance) or if one moves the phase along with the flow (phase covariance). In other words the problem sketched in Figure 10 does not occur.

In this section we shall consider general phase covariant convection in the Gabor domain. We shall consider both linear and non-linear left-invariant convection operators on Gabor transforms defined on the reduced Heisenberg group $H_{r}$. However, we recall that a linear left-invariant operator $\Phi$ on the space of Gabor transforms yields a linear net operator $\Upsilon_{\psi}$ on signals. Now such operator $\Upsilon_{\psi}$ commutes with an irreducible representation so that by the extended Schur's lemma for closed not necessarily bounded operators, [10] Vol.V p.21, it follows that $\Upsilon_{\psi}$ is constant. So for enhancement purposes in the signal domain these left-invariant convection equations with constant coefficient are useless, but they do provide an important preliminary point of view to the non-linear phase covariant convection equations.

We consider the following left-invariant evolution equations on the reduced Heisenberg group $H_{r}:$

$$
\left\{\begin{aligned}
\frac{\partial W}{\partial t}(g, t) & =-\sum_{i=1}^{2} v^{i}\left(\left|\mathcal{W}_{\psi} f\right|\right)(p, q) \mathcal{A}_{i} W(g, t), \quad g=\left(p, q, e^{2 \pi i s}\right) \in H_{r}, t>0 \\
W(g, 0) & =\mathcal{W}_{\psi} f(g) .
\end{aligned}\right.
$$

where the velocities $v^{i}\left(\mathcal{W}_{\psi} f\right)(g) \in \mathbb{C}$ are only dependent on the actual location $g$ in the group via the initial condition $\mathcal{W}_{\psi} f$. To ensure left-invariance of the operators $W(\cdot, 0) \mapsto W(\cdot, t)$ for all stopping times $t>0$ we will require

$$
v^{i}\left(\left|\mathcal{L}_{h} \mathcal{W}_{\psi} f\right|\right)(g)=\mathcal{L}_{h} v^{i}\left(\left|\mathcal{W}_{\psi} f\right|\right)(p, q)=v^{i}\left(\left|\mathcal{W}_{\psi} f\right|\right)\left(p-p^{\prime}, q-q^{\prime}\right),
$$

for all $f \in \mathbb{L}_{2}(\mathbb{R})$, and all $g=\left(p, q, e^{2 \pi i s}\right), h=\left(p^{\prime}, q^{\prime}, e^{2 \pi i s^{\prime}}\right) \in H_{r}$. Furthermore we will assume that $(p, q) \mapsto v^{i}\left(\mathcal{W}_{\psi} f\right)(p, q)$ are infinintely differentiable with bounded partial derivatives so that 
the ordinary differential equations

$$
\left\{\begin{array}{l}
\frac{d p}{d t}=-v^{1}\left(\left|\mathcal{W}_{\psi} f\right|\right)(p, q) \\
\frac{d q}{d t}=-v^{2}\left(\left|\mathcal{W}_{\psi} f\right|\right)(p, q) \\
\frac{d s}{d t}=\frac{q}{2} \frac{d p}{d s}-\frac{p}{2} \frac{d q}{d s} \\
(p(0), q(0), s(0))=\left(p_{0}, q_{0}, s_{0}\right)
\end{array}\right.
$$

have a unique solution which we shall denote by

$$
t \mapsto \gamma_{f}^{g_{0}}(t) \text { for all } g_{0}:=\left(p_{0}, q_{0}, t_{0}\right) \in H_{r} .
$$

Then by applying the method of characteristics on solving first order PDE's on manifolds we find the following unique solutions:

Theorem 6.10. Let $\psi$ be a rapidly decreasing infinitely differentiable function, i.e. $\psi \in \mathcal{S}(\mathbb{R})$. Let $(p, q) \mapsto v^{i}\left(\mathcal{W}_{\psi} f\right)(p, q), i=1,2$ be infinitely differentiable with bounded partial derivatives. Then the unique smooth solutions of the phase-covariant convection equations (6.57) are given by

$$
\begin{aligned}
W(g, t) & =\mathcal{G}_{\psi}(f)\left(\gamma_{f}^{g}(t)\right), \quad, t>0, g \in H_{r} \\
& =\mathcal{G}_{\psi}(f)\left(g \gamma_{\mathcal{U}_{g^{-1}} f}^{e}(t)\right) .
\end{aligned}
$$

The operator $\mathcal{G}_{\psi}(f) \mapsto W(\cdot, t)$ is phase covariant, which means that the phase moves along with the left-invariant flow:

$$
\arg \{W(g, t)\}=\arg \left\{\mathcal{G}_{\psi} f\right\}\left(\gamma_{f}^{g}(t)\right),
$$

for all stopping times $t>0$.

Proof. First we shall show that $g \gamma_{\mathcal{U}_{g-1} f}^{e}(t)=\gamma_{f}^{g}(t)$ for all $g \in H_{r}$ and all $t>0$ and all $f \in \mathbb{L}_{2}(\mathbb{R})$. To this end we note that both solutions are horizontal curves, i.e.

$$
\left\langle\left.\mathrm{d} \mathcal{A}^{3}\right|_{\gamma_{f}^{g}(t)}, \dot{\gamma}_{f}^{g}(t)\right\rangle=\left\langle\left.\mathrm{d} \mathcal{A}^{3}\right|_{g \gamma_{\mathcal{u}_{g^{-1}} f}^{e}(t)}, g \dot{\gamma}_{\mathcal{U}_{g^{-1}} f}^{e}(t)\right\rangle=0
$$

where $\mathrm{d} \mathcal{A}^{3}=\mathrm{d} s+2(p \mathrm{~d} q-q \mathrm{~d} p)$. So it is sufficient to check whether the first two components of the curves coincide. by

Now let $g=(p, q, s) \in H_{r}$ and define $p_{e}: \mathbb{R}^{+} \rightarrow \mathbb{R}, q_{e}: \mathbb{R}^{+} \rightarrow \mathbb{R}$ and $p_{g}: \mathbb{R}^{+} \rightarrow \mathbb{R}, q_{g}: \mathbb{R}^{+} \rightarrow \mathbb{R}$

$$
\begin{array}{ll}
p_{e}(t):=\left\langle\mathrm{d} p, g \dot{\gamma}_{\mathcal{U}_{g^{-1}} f}^{e}(t)\right\rangle, & p_{g}(t):=\left\langle\mathrm{d} p, \dot{\gamma}_{f}^{g}(t)\right\rangle, \\
q_{e}(t):=\left\langle\mathrm{d} q, g \dot{\gamma}_{\mathcal{U}_{g^{-1}} f}^{e}(t)\right\rangle, & q_{g}(t):=\left\langle\mathrm{d} q, \dot{\gamma}_{f}^{g}(t)\right\rangle,
\end{array}
$$

then it remains to be shown that $p_{e}+p=p_{g}$ and $p_{e}+q=q_{g}$. To this end we compute

$$
\begin{aligned}
\frac{d p_{e}}{d t}(t) & =v^{1}\left(\left|\mathcal{W}_{\psi} \mathcal{U}_{g^{-1}} f\right|\right)\left(p_{e}(t), q_{e}(t)\right) \\
& =v^{1}\left(\left|\mathcal{L}_{g^{-1}} \mathcal{W}_{\psi} f\right|\right)\left(p_{e}(t), q_{e}(t)\right) \\
& =v^{1}\left(\left|\mathcal{W}_{\psi} f\right|\right)\left(p_{e}(t)+p, q_{e}(t)+q\right),
\end{aligned}
$$

so that we see that $\left(p_{e}+p, q_{e}+q\right)$ satisfies the following ODE system:

$$
\left\{\begin{array}{lll}
\frac{d}{d t}\left(p+p_{e}\right)(t)=v^{1}\left(\left|\mathcal{W}_{\psi} f\right|\right)\left(p_{e}(t)+p, q_{e}(t)+q\right), & t>0 \\
\frac{d}{d t}\left(q+q_{e}\right)(t)=v^{2}\left(\left|\mathcal{W}_{\psi} f\right|\right)\left(q_{e}(t)+q, q_{e}(t)+q\right), & t>0 \\
p+p_{e}=p & & \\
q+q_{e}=q &
\end{array}\right.
$$


now this initial value problem has a unique smooth solution, so indeed $p_{g}=p+p_{e}$ and $q_{g}=q+q_{e}$. Furthermore we have by means of the chain-rule for differentiation:

$$
\begin{aligned}
\frac{d}{d t}\left(\mathcal{W}_{\psi} f\right)\left(\gamma_{f}^{g}(t)\right)= & \sum_{i=1}^{2}\left\langle\left.\mathrm{~d} \mathcal{A}^{i}\right|_{\gamma_{f}^{g}(t)}, \dot{\gamma}_{f}^{g}(t)\right\rangle\left(\left.\mathcal{A}_{i}\right|_{\gamma_{f}^{g}(t)} \mathcal{W}_{\psi} f\right)\left(\gamma_{f}^{g}(t)\right) \\
= & \left.\dot{p}^{g}(t) \mathcal{A}_{1}\right|_{\gamma_{f}^{g}(t)} \mathcal{W}_{\psi} f\left(\gamma_{f}^{g}(t)\right)+\left.\dot{q}^{g}(t) \mathcal{A}_{2}\right|_{\gamma_{f}^{g}(t)} \mathcal{W}_{\psi} f\left(\gamma_{f}^{g}(t)\right) \\
= & -\left.v^{1}\left(\left|\mathcal{W}_{\psi} f\right|\right)\left(p^{g}(t), q^{g}(t)\right) \mathcal{A}_{1}\right|_{\gamma_{f}^{g}(t)} \mathcal{W}_{\psi}\left(\gamma_{f}^{g}(t)\right) \\
& \quad-\left.v^{2}\left(\left|\mathcal{W}_{\psi} f\right|\right)\left(p^{g}(t), q^{g}(t)\right) \mathcal{A}_{2}\right|_{\gamma_{f}^{g}(t)} \mathcal{W}_{\psi}\left(\gamma_{f}^{g}(t)\right)
\end{aligned}
$$

The corresponding left-invariant evolution equations on phase space are given by

$$
\left\{\begin{array}{l}
\frac{\partial W}{\partial t}(p, q, t)=-\sum_{i=1}^{2} \tilde{v}^{i}\left(\left|\mathcal{G}_{\psi} f\right|\right)(p, q) \tilde{\mathcal{A}}_{i} W(p, q, t) \\
W(p, q, 0)=\mathcal{G}_{\psi} f(p, q)
\end{array}\right.
$$

where $\tilde{v}^{i}\left(\left|\mathcal{G}_{\psi} f\right|\right)(p, q)=v^{i}\left(\left|\mathcal{W}_{\psi}(f)\right|\right)(p, q)$.

Now first consider the linear case where $v^{i}=$ constant, $i=1,2$. Then we have for each stopping time $t>0$

$$
\begin{aligned}
& \Phi_{t}\left(\mathcal{W}_{\psi} f\right):=W(g, t)=\left(e^{t\left(v^{1} \mathcal{A}_{1}+v^{2} \mathcal{A}_{2}\right)} \mathcal{W}_{\psi} f\right)(g) \\
& =\left(\mathcal{W}_{\psi} f\right)\left(g e^{t v^{i} A_{i}}\right)=\left(\mathcal{U}_{g} \mathcal{U}_{e^{t A_{i}}} \psi, f\right)_{\mathbb{L}_{2}(\mathbb{R})}=\mathcal{W}_{\mathcal{U}_{e} t A_{i}} f
\end{aligned}
$$

so consequently, by Schur's lemma, the net operator $\Upsilon_{t, \psi}=\mathcal{W}_{\psi}^{*} \circ \Phi_{t} \circ \mathcal{W}_{\psi}$ on the image is a multiple of the identity and indeed one has

$$
\Upsilon_{t, \psi} f=\mathcal{W}_{\psi}^{*} \mathcal{W}_{\mathcal{U}_{e} A_{i}} \psi f=\left(\psi, \mathcal{U}_{e^{t A_{i}}} \psi\right) f .
$$

Despite the obvious fact the net operator is a highly trivial operator on a signal, we can make an important observation: Horizontal (i.e. $v_{3}=0$ ), linear left-invariant convection along takes place along the parallel exponential curves:

$$
\gamma^{g_{0}}(t)=g_{0} e^{t\left(v^{1} A_{1}+v^{2} A_{2}\right)}=g_{0}\left(t v^{1}, t v^{2}, 0\right)=\left(p_{0}+t v^{1}, q_{0}+t v^{2}, t_{0}+\frac{t}{2}\left(v^{1} q_{0}-v^{2} p_{0}\right)\right) .
$$

for all $g_{0}=\left(p_{0}, q_{0}, t_{0}\right) \in H_{r}$. In practice it does not make sense to transport everything along parallel exponential curves.

\section{Left Invariant Diffusion on Gabor transforms}

Before we shall consider left-invariant adaptive diffusion on Gabor transforms, we shall provide a quick review on adaptive left-invariant diffusions on signals/images, which we shall generalize to left-invariant adaptive diffusions on Gabor transforms where we consistently replace the domain of signals, i.e. the additive group $\mathbb{R}^{d}$ by the domain of the Gabor transforms, i.e. the Weyl-Heisenberg group $H_{r}$.

A scale space representation $u_{f}: \mathbb{R}^{d} \times \mathbb{R}^{+} \rightarrow \mathbb{R}$ of a signal/image $f: \mathbb{R}^{d} \rightarrow \mathbb{R}$ is usually obtained by solving an evolution equation on the additive group $\left(\mathbb{R}^{d},+\right)$, with usually $d \in\{1,2,3\}$. The most common evolution equation, in image analysis, is the diffusion equation,

$$
\left\{\begin{array}{l}
\partial_{s} u_{f}(\mathbf{x}, s)=\nabla_{\mathbf{x}} \cdot\left(C\left(u_{f}\right)(\mathbf{x}, s) \nabla_{\mathbf{x}} u_{f}\right)(\mathbf{x}, s) \\
u_{f}(\mathbf{x}, 0)=f(\mathbf{x}),
\end{array}\right.
$$

where $\left.C: \mathbb{L}_{2}\left(\mathbb{R}^{2} \times \mathbb{R}^{+}\right) \cap C^{2}\left(\mathbb{R}^{2} \times \mathbb{R}^{+}\right) \rightarrow C^{1}\left(\mathbb{R}^{2} \times \mathbb{R}^{+}\right)\right)$is a function which takes care of adaptive conductivity, that is conductivity depending on the local differential structure at $\left(\mathbf{x}, s, u_{f}(\mathbf{x}, s)\right)$. In case $C=1$, this local differential structure is not used and the solution is given by convolution

$$
u_{f}(\mathbf{x}, s)=\left(G_{s} * f\right)(\mathbf{x})=\int_{\mathbb{R}^{d}} G_{s}(\mathbf{x}-\mathbf{y}) f(\mathbf{y}) \mathrm{d} \mathbf{y},
$$


with a Gaussian kernel $G_{s}(\mathbf{x})=\frac{1}{(4 \pi s)^{\frac{d}{2}}} e^{-\frac{\|\mathbf{x}\|}{4 s}}$ with scale, $s=\frac{1}{2} \sigma^{2}>0$.

As pointed out by Perona and Malik [37], nonlinear image adaptive isotropic diffusion is achieved by replacing $C=1$ by $C\left(u_{f}\right)(\mathbf{x}, s)=c\left(\left\|\nabla_{\mathbf{x}} u_{f}(\mathbf{x}, s)\right\|\right)$, where $c: \mathbb{R}^{+} \rightarrow \mathbb{R}^{+}$is some smooth strictly decaying positive function vanishing at infinity. This is based on the idea that if (locally) the gradient is large you do not want to diffuse too much. By restricting ourselves to positively valued $c>0$ one ensures that the diffusion is always forward, and thereby ill-posed backward diffusion is avoided. The common choices are

$$
c(t)=e^{-\frac{c}{\left(\frac{\lambda}{t}\right)^{2 p}}}, \quad c(t)=\frac{1}{\left(\frac{t}{\lambda}\right)^{2 p}+1} \text { and } c(t)=\frac{1}{\sqrt{\left(\frac{t}{\lambda}\right)^{2}+1}},
$$

involving parameters $p>\frac{1}{2}, \lambda>0$.

A further improvement of the Perona and Malik scheme is introduced by Weickert [46], who also uses the direction of the gradient $\nabla_{\mathbf{x}} u_{f}$ of $u_{f}$, which is not used in the algorithms of Perona and Malik type. He proposed "coherence enhancing diffusion" (CED) where the diffusion constant $c$ is replaced by a diffusion matrix:

$$
\begin{aligned}
& S\left(u_{f}\right)(\mathbf{x}, s)=\left(G_{\sigma} * \nabla u_{f}(\cdot, s)\left(\nabla u_{f}(\cdot, s)\right)^{T}\right)(\mathbf{x}), \\
& C\left(u_{f}\right)(\mathbf{x}, s)=\varepsilon I+(1-\varepsilon) e^{-\frac{c}{\left(\lambda_{1}\left(S\left(u_{f}\right)(\mathbf{x}, s)\right)-\lambda_{2}\left(S\left(u_{f}\right)(\mathbf{x}, s)\right)\right)^{2}}} \mathbf{e}_{2}\left(S\left(u_{f}\right)(\mathbf{x}, s)\right) \mathbf{e}_{2}^{T}\left(S\left(u_{f}\right)(\mathbf{x}, s)\right)
\end{aligned}
$$

where $\varepsilon \in(0,1), c>0, \sigma>0$ are parameters and where the so-called "structure tensor" $S$, with eigenvalues $\left\{\lambda_{i}\left(S\left(u_{f}\right)(\mathbf{x}, s)\right)\right\}_{i=1,2}$ is used to get a measure for local anisotropy $e^{-\frac{\left.\lambda_{1}\left(S\left(u_{f}\right)(\mathbf{x}, s)\right)-\lambda_{2}\left(S\left(u_{f}\right)(\mathbf{x}, s)\right)\right)^{2}}{\left(\lambda^{2}\right.}}$ together with an orientation estimate $\mathbf{e}_{2}\left(S\left(u_{f}\right)(\mathbf{x}, s)\right)$, which is the eigenvector of the structure tensor with smallest eigenvalue. In order to get robust orientation estimates it is essential to apply a componentwise smoothing on the so-called "structure-tensor field" $\nabla u_{f} \otimes \nabla u_{f}$.

In stead of this structure tensor field it is also common to consider the Hessian field $\mathbf{x} \mapsto$ $H u_{f}(\mathbf{x}, s)=G_{\sigma} * H f(\mathbf{x}), s=\frac{1}{2} \sigma^{2}$, whose matrix components are given by

$$
\mathbf{x} \mapsto G_{\sigma} * H f(\mathbf{x})=\left[\left(G_{\sigma} * \partial_{x_{i}} \partial_{x_{j}} f(\cdot)\right)(\mathbf{x})\right]_{i, j=1,2}=\left[\left(\partial_{x_{i}} \partial_{x_{j}} G_{\sigma} * f\right)(\mathbf{x})\right]_{i, j=1,2} .
$$

A clear advantage over the smoothed structure tensor field $G_{\sigma} * \nabla u_{f} \otimes \nabla u_{f}$ is that the Hessian field of a natural image/signal is almost everywhere invertible as $\sigma \downarrow 0$, whereas the structure tensor field is everywhere not invertible as $\sigma \downarrow 0$. A slight drawback of using the second order differential structure, rather than the first order structure at $(\mathbf{x}, s) \mapsto u_{f}(\mathbf{x}, s)$ is that one requires a higher degree of regularity, making the algorithm less robust to noise. Finally, we note that the eigen vector with smallest eigenvalue of $H u_{f}(\mathbf{x}, s)$ typically points along lines in images whereas the vector orthogonal to the spatial gradient $\nabla u_{f}(\mathbf{x}, s)=\nabla_{\mathbf{x}} u_{f}(\mathbf{x}, s)$ typically points along edges in images.

In order to generalize the CED (coherence enhancing diffusion) schemes to Gabor transforms we simply have to replace the left-invariant vector fields $\left\{\partial_{x}, \partial_{y}\right\}$ on the additive group $\left(\mathbb{R}^{2},+\right)$, by the left-invariant vector fields on $H_{r}$. To this end we formulate the standard coherence enhancing diffusion equations on images (with conductivity (7.61)) as

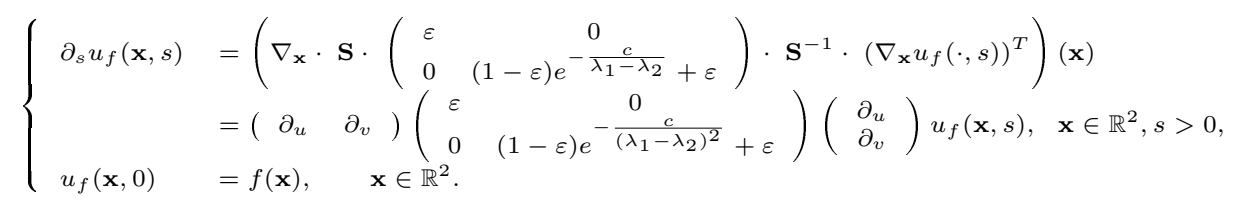

Here we expressed the diffusion equations in both the global standard basis $\left\{\mathbf{e}_{x}, \mathbf{e}_{y}\right\}:=\{(1,0),(0,1)\} \leftrightarrow$ $\left\{\partial_{x}, \partial_{y}\right\}$ and in the locally adapted basis of eigen vectors of auxiliary matrix $S\left(u_{f}(\cdot, s)\right)(\mathbf{x})$ (in image analysis known as the "structure tensor", recall (7.61)):

$$
\left\{\mathbf{e}_{1}, \mathbf{e}_{2}\right\}:=\left\{\mathbf{e}_{1}\left(S\left(u_{f}(\cdot, s)\right)(\mathbf{x})\right), \mathbf{e}_{2}\left(S\left(u_{f}(\cdot, s)\right)(\mathbf{x})\right)\right\} \leftrightarrow\left\{\partial_{u}, \partial_{v}\right\},
$$

with respective eigen values $\lambda_{k}:=\lambda_{k}\left(S\left(u_{f}(\cdot, s)\right)(\mathbf{x})\right), k=1,2$. The corresponding orthogonal basis transform which maps the standard basis vectors to the eigenvectors $\left\{\mathbf{e}_{1}, \mathbf{e}_{2}\right\}$ is denoted by 

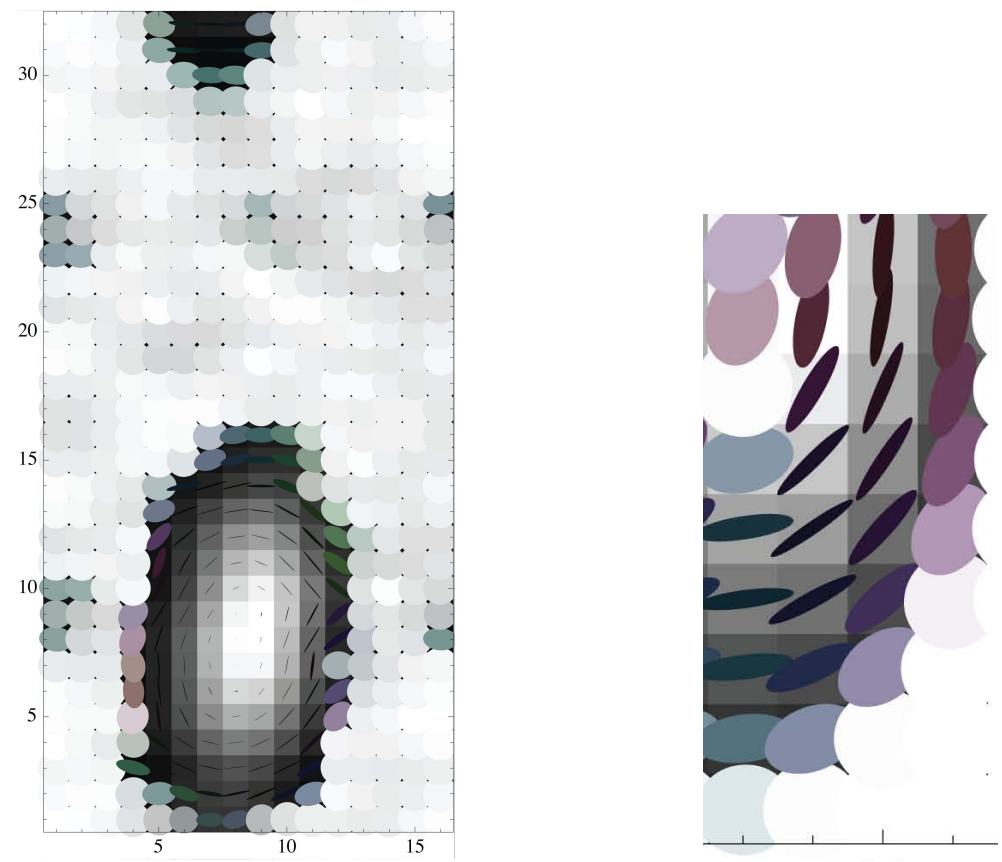

Figure 11: Illustration of the adaptive left-invariant diffusions. On top of $(p, q) \mapsto\left|W_{\psi}(p, q, s, t)\right|$ we plot ellipsoids representing the local eigen-vectors of the Hessian-form along which the diffusion locally takes place. The directions of the ellipsoids coincide with the directions of the Hessian, whereas the anisotropy of the ellipsoids is determined by the fraction $\left|\lambda_{1} / \lambda_{2}\right|$ of the eigen values $\left\{\lambda_{1}, \lambda_{2}\right\}$ with $\left|\lambda_{1}\right|>\left|\lambda_{2}\right|$, the colors indicate the directions of the largest eigen-vector and the intensity reflects the relative damping factor $(p, q) \mapsto 1+\epsilon^{-1}(1-\epsilon) e^{-c\left(\lambda_{1}(p, q)-\lambda_{2}(p, q)\right)^{-2}} \lambda_{1}(p, q)$ of the second eigen vector. Left: Ellipsoids reflecting the diffusion in Figure 2 (third column). Right: typical fragment of the ellipsoids for different parameter-value $c>0$.

$\mathbf{S}=\left(\mathbf{e}_{1} \mid \mathbf{e}_{2}\right)$ and we have $\left(\begin{array}{ll}\partial_{u} & \partial_{v}\end{array}\right)=\left(\begin{array}{ll}\partial_{x} & \partial_{y}\end{array}\right) \cdot \mathbf{S}$. At isotropic areas $\lambda_{1} \rightarrow \lambda_{2}$ and thereby the conductivity matrix becomes a multiple of the identity yielding isotropic diffusion only at isotropic areas, which is desirable for noise-removal.

Now we replace the $2 \mathrm{D}$-signal by the Gabor transform of a $1 D$-signal, the group $\mathbb{R}^{2}$ by $H_{r}$, the standard inner product on $\mathbb{R}^{2}$ by the first fundamental form (C.74) parameterized by $\beta$, where the parameter $\beta^{-1}$ has physical dimension length, so that the first fundamental form is consistent with physical dimensions. Finally, we replace the basis of normalized left invariant vector fields $\left\{\mathbf{e}_{x}, \mathbf{e}_{y}\right\}:=\{(1,0),(0,1)\} \leftrightarrow\left\{\partial_{x}, \partial_{y}\right\}$ on $\mathbb{R}^{2}$ by the normalized left invariant vector fields $\left\{\mathbf{e}_{x}, \mathbf{e}_{y}\right\}:=\{(1,0),(0,1)\} \leftrightarrow\left\{\beta^{-2} \mathcal{A}_{1}, \mathcal{A}_{2}\right\}$ on $H_{r}$, so that we arrive at the following system for adaptive horizontal left invariant diffusion on Gabor transforms:

$$
\left\{\begin{aligned}
& \partial_{t} W(p, q, s, t)=\left(\begin{array}{ll}
\left.\left(\begin{array}{ll}
\beta^{-2} \mathcal{A}_{1} & \mathcal{A}_{2}
\end{array}\right) \cdot \mathbf{S} \cdot\left(\begin{array}{cc}
\varepsilon & 0 \\
0 & (1-\varepsilon) e^{-\frac{c}{\lambda_{1}-\lambda_{2}}}+\varepsilon
\end{array}\right) \cdot \mathbf{S}^{-1}\left(\begin{array}{c}
\beta^{-2} \mathcal{A}_{1} W \\
\mathcal{A}_{2} W
\end{array}\right)\right)(p, q, s, t) \\
& =\left(\begin{array}{ll}
\partial_{u} & \partial_{v}
\end{array}\right)\left(\begin{array}{cc}
\varepsilon & (1-\varepsilon) e^{-\frac{c}{\left(\lambda_{1}-\lambda_{2}\right)^{2}}}+\varepsilon
\end{array}\right)\left(\begin{array}{c}
\partial_{u} \\
\partial_{v}
\end{array}\right) W(p, q, s, t), \\
& \text { for all }(p, q, s) \in H_{r}, t>0 \text { and fixed } c>0, \varepsilon>0,
\end{array}\right. \\
& W(p, q, s, 0) \quad=\mathcal{W}_{\psi} f(p, q, s), \text { for all }(p, q, s) \in H_{r} .
\end{aligned}\right.
$$

with $\mathbf{S}=\left(\mathbf{e}_{1} \mid \mathbf{e}_{2}\right)$ and $\left(\partial_{u} W \quad \partial_{v} W\right)=\left(\beta^{-2} \mathcal{A}_{1} W \mathcal{A}_{2} W\right) \mathbf{S}$ and where

$$
\begin{aligned}
& \lambda_{k}:=\lambda_{k}\left(S\left(\left|\mathcal{W}_{\psi} f\right|\right)(p, q, s)\right), k=1,2,\left|\lambda_{1}\right| \leq\left|\lambda_{2}\right| \\
& \mathbf{e}_{k}:=\mathbf{e}_{k}\left(S\left(\left|\mathcal{W}_{\psi} f\right|\right)(p, q, s)\right), k=1,2,
\end{aligned}
$$

denote the eigenvalues and $\left\{\mathbf{e}_{1}, \mathbf{e}_{2}\right\} \leftrightarrow\left\{\partial_{u}, \partial_{v}\right\}$ the corresponding normalized eigen vectors of a 
local auxiliary matrix $S\left(\left|\mathcal{W}_{\psi} f\right|\right)(p, q, s)$ :

$$
S\left(\left|\mathcal{W}_{\psi} f\right|\right)(p, q, s) \mathbf{e}_{k}=\lambda_{k} \mathbf{e}_{k} .
$$

Here the normalization of the eigen vectors is with respect to the first fundamental form (C.74) parameterized by $\beta$, i.e.

$$
\begin{aligned}
& \mathcal{G}_{\beta}\left(\partial_{u}, \partial_{u}\right)=\mathcal{G}_{\beta}\left(\partial_{v}, \partial_{v}\right)=\mathcal{G}_{\beta}\left(\beta^{-2} \mathcal{A}_{1}, \beta^{-2} \mathcal{A}_{1}\right)=\mathcal{G}_{\beta}\left(\mathcal{A}_{2}, \mathcal{A}_{2}\right)=1 \quad \leftrightarrow \\
& \mathbf{e}_{1}^{T} \mathbf{e}_{1}=\mathbf{e}_{2}^{T} \mathbf{e}_{2}=\mathbf{e}_{x}^{T} \mathbf{e}_{x}=\mathbf{e}_{y}^{T} \mathbf{e}_{y}=1 \Rightarrow \mathbf{S}^{T}=\mathbf{S}^{-1} .
\end{aligned}
$$

This auxiliary matrix $S\left(\left|\mathcal{W}_{\psi} f\right|\right)(p, q, s)$ at each position $(p, q, s) \in H_{r}$ is chosen such that it depends only on the absolute value $\left|\mathcal{W}_{\psi} f\right|$ (so it is independent of phase parameter $s$ ) of the initial condition and so does the corresponding conductivity matrix-valued function:

$$
C=\mathbf{S} \cdot\left(\begin{array}{cc}
\varepsilon & 0 \\
0 & (1-\varepsilon) e^{-\frac{c}{\lambda_{1}-\lambda_{2}}}+\varepsilon
\end{array}\right) \cdot \mathbf{S}^{-1} .
$$

We propose the following specific choices of auxiliary matrices ${ }^{8}$

$$
\begin{aligned}
& S\left(\left|\mathcal{W}_{\psi} f\right|\right)(p, q, s)=\left[\left(\partial_{p_{i}} \partial_{p_{j}} G_{\sigma} *\left|\mathcal{W}_{\psi} f\right|\right)(p, q)\right]_{i, j=1,2}, \\
& S\left(\left|\mathcal{W}_{\psi} f\right|\right)(p, q, s)=\left[\left(\partial_{p_{i}} G_{\sigma} *\left|\mathcal{W}_{\psi} f\right|\right)(p, q)\left(\partial_{p_{j}} G_{\sigma} *\left|\mathcal{W}_{\psi} f\right|\right)(p, q)\right]_{i, j=1,2}
\end{aligned}
$$

with $p_{1}=\beta^{2} p$ and $p_{2}=q$ and separable Gaussian kernel

$$
G_{\sigma}(p, q)=\frac{\beta^{2}}{2 \pi \sigma^{2}} e^{-\frac{\beta^{4} p^{2}+q^{2}}{2 \sigma^{2}}} .
$$

Now since $\left|\mathcal{W}_{\psi} f\right|=\left|\mathcal{G}_{\psi} f\right|$ and $\tilde{A}_{i}=\mathcal{S} \circ \mathcal{A}_{i} \circ \mathcal{S}^{-1}$ (recall $(2.23)$ ) and $\mathcal{S} \circ \mathcal{W}_{\psi} f=\mathcal{G}_{\psi} f$ we get the following equivalent non-linear left-invariant diffusion equations on phase space:

$$
\begin{aligned}
& \left(\partial_{t} \tilde{W}(p, q, t)=\left(\begin{array}{cc}
\beta^{-2} \tilde{\mathcal{A}}_{1} & \tilde{\mathcal{A}}_{2}
\end{array}\right) \cdot \mathbf{S} \cdot\left(\begin{array}{cc}
\varepsilon & 0 \\
0 & (1-\varepsilon) e^{-\frac{c}{\lambda_{1}-\lambda_{2}}}+\varepsilon
\end{array}\right) \cdot \mathbf{S}^{-1}\left(\begin{array}{c}
\beta^{-2} \tilde{\mathcal{A}}_{1} \tilde{W} \\
\tilde{\mathcal{A}}_{2} \tilde{W}
\end{array}\right)\right)(p, q, t) \\
& =\left(\begin{array}{ll}
\partial_{\tilde{u}} & \partial_{\tilde{v}}
\end{array}\right)\left(\begin{array}{cc}
\varepsilon & 0 \\
0 & (1-\varepsilon) e^{-\frac{c}{\left(\lambda_{1}-\lambda_{2}\right)^{2}}}+\varepsilon
\end{array}\right)\left(\begin{array}{c}
\partial_{\tilde{u}} \\
\partial_{\tilde{v}}
\end{array}\right) W(p, q, t), \\
& \text { for all }(p, q) \in \mathbb{R}^{2}, t>0 \text { and fixed } c>0, \varepsilon>0 \text {, } \\
& \tilde{W}(p, q, 0) \quad=\mathcal{G}_{\psi} f(p, q) \text {, for all }(p, q) \in \mathbb{R}^{2} .
\end{aligned}
$$

with $\mathbf{S}=\left(\mathbf{e}_{1} \mid \mathbf{e}_{2}\right)$ and $\left(\partial_{a} W \quad \partial_{b} W\right)=\left(\beta^{-2} \mathcal{A}_{1} W \mathcal{A}_{2} W\right) \mathbf{S}$ and where

$$
\begin{aligned}
& \lambda_{k}:=\lambda_{k}\left(S\left(\left|\mathcal{G}_{\psi} f\right|\right)(\mathbf{x}, s)\right), k=1,2, \\
& \mathbf{e}_{k}:=\mathbf{e}_{k}\left(S\left(\left|\mathcal{G}_{\psi} f\right|\right)(\mathbf{x}, s)\right), k=1,2,
\end{aligned}
$$

denote the eigenvalues and $\left\{\mathbf{e}_{1}, \mathbf{e}_{2}\right\}$ the corresponding normalized eigen vectors of a local helpmatrix $S\left(\left|\mathcal{G}_{\psi} f\right|\right)(\mathbf{x}, s)=S\left(\left|\mathcal{W}_{\psi} f\right|\right)(\mathbf{x}, s)$. Now, these eigen vectors in $\mathbb{R}^{2}$ correspond to

$$
\left\{\mathbf{e}_{1}, \mathbf{e}_{2}\right\} \leftrightarrow\left\{\partial_{\tilde{u}}, \partial_{\tilde{v}}\right\}=\left\{\mathcal{S} \circ \partial_{u} \circ \mathcal{S}^{-1}, \mathcal{S} \circ \partial_{v} \circ \mathcal{S}^{-1}\right\},
$$

so that we indeed get the right correspondence between (7.64) and (7.63):

$$
\begin{aligned}
& \forall(p, q, s) \in H_{r}, t>0 \quad: \quad \partial_{t} W(p, q, s, t)=\left(\begin{array}{cc}
\partial_{u} & \partial_{v}
\end{array}\right)\left(\begin{array}{cc}
\varepsilon & 0 \\
0 & (1-\varepsilon) e^{-\frac{0}{\left(\lambda_{1}-\lambda_{2}\right)^{2}}+\varepsilon}
\end{array}\right)\left(\begin{array}{l}
\partial_{u} \\
\partial_{v}
\end{array}\right) W(p, q, s, t) \Leftrightarrow \\
& \forall(p, q, s) \in H_{r}, t>0 \quad: \quad \partial_{t}\left(\mathcal{S}^{-1} \tilde{W}\right)(p, q, s, t)=\left(\begin{array}{cc}
\partial_{u} & \partial_{v}
\end{array}\right)\left(\begin{array}{cc}
\varepsilon & 0 \\
0 & (1-\varepsilon) e^{-\frac{c}{\left(\lambda_{1}-\lambda_{2}\right)^{2}}+\varepsilon}
\end{array}\right)\left(\begin{array}{c}
\partial_{u} \\
\partial_{v}
\end{array}\right)\left(\mathcal{S}^{-1} \tilde{W}\right)(p, q, s, t) \Leftrightarrow \\
& \forall(p, q, s) \in H_{r}, t>0 \quad: \quad \partial_{t}\left(\mathcal{S}^{-1} \tilde{W}\right)(p, q, s, t)=\left(\begin{array}{cc}
\partial_{u} & \partial_{v}
\end{array}\right) \mathcal{S}^{-1}\left(\begin{array}{cc}
\varepsilon & 0 \\
0 & (1-\varepsilon) e^{-\frac{c}{\left(\lambda_{1}-\lambda_{2}\right)^{2}}}+\varepsilon
\end{array}\right) \mathcal{S}\left(\begin{array}{c}
\partial_{u} \\
\partial_{v}
\end{array}\right)\left(\mathcal{S}^{-1} \tilde{W}\right)(p, q, s, t) \Leftrightarrow \\
& \forall_{(p, q) \in \mathbb{R}^{2}, t>0}: \quad \partial_{t} \tilde{W}(p, q, t)=\left(\begin{array}{cc}
\partial_{\tilde{u}} & \partial_{\tilde{v}}
\end{array}\right)\left(\begin{array}{cc}
\varepsilon & 0 \\
0 & (1-\varepsilon) e^{-\frac{c}{\left(\lambda_{1}-\lambda_{2}\right)^{2}}}+\varepsilon
\end{array}\right)\left(\begin{array}{c}
\partial_{\tilde{u}} \\
\partial_{\tilde{v}}
\end{array}\right) \tilde{W}(p, q, t)
\end{aligned}
$$

\footnotetext{
${ }^{8}$ Note that $\mathcal{A}_{1}\left|\mathcal{W}_{\psi} f\right|=\partial_{p}\left|\mathcal{W}_{\psi} f\right|$ and $\mathcal{A}_{2}\left|\mathcal{W}_{\psi} f\right|=\partial_{q}\left|\mathcal{W}_{\psi} f\right|$.
} 
so that the uniqueness of the solutions $\tilde{W}$ of (7.64) and $W$ of (7.63) implies that

$$
\tilde{W}(\cdot, \cdot, 0)=\mathcal{G}_{\psi} f=\mathcal{S} \circ \mathcal{W}_{\psi} f=\mathcal{S} \circ W(\cdot, \cdot, \cdot, 0) \Rightarrow \forall_{t \geq 0}: \tilde{W}(\cdot, \cdot, t)=\mathcal{S} \circ W(\cdot, \cdot, \cdot, t) .
$$

Now it is clear that problem (7.64) is preferable over problem (7.63) if it comes to numerical schemes (such as a simple Euler forward finite discretization scheme, using the left-invariant finite differences 4.49), since it is an essentially $2 D$-diffusion problem, whereas (7.63) is a $3 D$-problem.

We implemented the diffusions (7.64) by a standard explicit Euler-Forward scheme. Figure 2 shows a promising result of adaptive phase-covariant diffusions on an explicit example. For illustration of the local adaptivity of the phase-covariant diffusions in phase space (7.64) within this specific example, see Figure 11.

\section{A Co-Adjoint orbits and Fourier transform on $H_{3}$}

The adjoint action of the Heisenberg group on its own Lie-algebra is given by

$$
[\operatorname{Ad}(p, q, s)]\left(a^{1}, a^{2}, a^{3}\right)=\left(a^{1}, a^{2}, a^{3}+p a^{2}-q a^{1}\right),
$$

where $\left(a^{1}, a^{2}, a^{3}\right)$ represents the element $\sum_{i=1}^{3} a^{i} A_{i}=a^{1} \partial_{p}+a^{2} \partial_{q}+a^{3} \partial_{s}$ in the Lie-algebra $T_{e}\left(H_{3}\right)$. Note that this adjoint action is isomorphic to the adjoint action of $H_{3}$ on the Lie-algebra of left-invariant vector fields

$$
\operatorname{Ad}(p, q, s)\left(\sum_{i=1}^{3} a^{i} \mathcal{A}_{i}\right)=a^{1} \mathcal{A}_{1}+a^{2} \mathcal{A}_{2}+\left(a^{3}+p a^{2}-q a^{1}\right) \mathcal{A}_{3},
$$

and recall that $\operatorname{Ad}_{g}=\left(\mathcal{L}_{g} \mathcal{R}_{g^{-1}}\right)_{*}$, where $L_{g} h=g h$ and $R_{g} h=h g$.

By duality, the co-adjoint action of the Heisenberg group on the dual of its own Lie algebra is now given by

$$
[\operatorname{CoAd}(p, q, s)]\left(a_{1}, a_{2}, a_{3}\right)=\left(a_{1}+a_{3} p, a_{2}-a_{3} q, a_{3}\right) .
$$

So the coadjoint orbits are simply given by the planes $a_{3}=\lambda$ is constant and the singleton sets $\left\{a_{1}, a_{2}\right\}$ with zero measure.

As the Heisenberg group is a nilpotent simply connected Lie-group we may apply the Kirillov co-adjoint orbit theory, which relates the coadjoint orbits to all irreducible representations: The dual group $\hat{H}_{3}$ can be identified with the quotient $\mathbb{R}^{3}=\left\{a_{1}, a_{2}, a_{3} \mid a_{1}, a_{2}, a_{3} \in \mathbb{R}\right\}$ and the coadoint action.

All irreducible representations of $H_{3}$ are given by the representations (2.15), indexed by $\lambda \in \mathbb{R}$, $\lambda \neq 0$, corresponding to the planes $a_{3}=\lambda \neq 0$ and the representations $\pi_{a_{1}, a_{2}}: H_{3} \rightarrow \mathbb{C}$ given by

$$
\pi_{a_{1}, a_{2}}(p, q, s)=e^{2 \pi i\left(a_{1} p+a_{2} q\right)}
$$

corresponding to each singleton in the plane $\lambda=0$. Now the Plancherel measure $\mu_{\hat{H}_{3}}$ on the dual group $\hat{H}_{3}$ vanishes on the representations (A.65) and yields

$$
\mathrm{d} \mu_{\hat{H}_{3}}\left(\rho_{h}\right)=|\lambda| \mathrm{d} \lambda
$$

if applied to the representations (2.15) indexed by scaling parameter $\lambda \in \mathbb{R} \backslash\{0\}$. Now by identifying the domain of all irreducible representations and identifying $\lambda \leftrightarrow \mathcal{U}^{\lambda}$, we can define the Fourier transform on $H_{3}$ as an operator $\mathcal{F}_{H_{3}}: \mathbb{L}_{2}\left(H_{3}\right) \rightarrow \mathbb{L}_{2}\left(T_{2}\left(\mathbb{L}_{2}(\mathbb{R})\right), \lambda \mathrm{d} \lambda\right)$, where $T_{2}\left(\mathbb{L}_{2}(\mathbb{R})\right)=\left\{A \in \mathcal{B}\left(\mathbb{L}_{2}(\mathbb{R})\right) \mid\|A\|_{2}^{2}:=\operatorname{trace}\left\{A^{*} A\right\}<\infty\right\}$ as follows:

$$
[\mathcal{F}(W)](\lambda)=\int_{H_{3}} W(g) \mathcal{U}_{g^{-1}}^{\lambda} \mathrm{d} \mu_{H_{3}}(g) .
$$


Now for each $\lambda$ the operator $[\mathcal{F}(W)](\lambda)$ is a Hilbert-Schmidt operator, since

$$
\begin{aligned}
{\left[\left[\mathcal{F}_{H_{3}} W\right](\lambda) \phi\right](y) } & =\int_{H_{3}} W(g) \mathcal{U}_{g^{-1}}^{\lambda} \phi(y) \mathrm{d} \mu_{H_{3}}(g) \\
& =\int_{\mathbb{R}^{3}} W(p, q, s) \mathcal{U}_{-p,-q,-s}^{\lambda} \phi(y) \mathrm{d} p \mathrm{~d} q \mathrm{~d} s \\
& =\left(K_{W}^{\lambda}(y, \cdot), \phi\right)_{\mathbb{L}_{2}(\mathbb{R})}
\end{aligned}
$$

with square integrable kernel $K_{W}^{\lambda}: \mathbb{R}^{2} \rightarrow \mathbb{C}$ given by

$$
K_{W}^{\lambda}(y, p)=2 \pi \mathcal{F}[W(p-y, \cdot, \cdot)](-(1 / 2) \lambda(y+p), 2 \pi \lambda) \quad(y, p) \in \mathbb{R}^{2},
$$

where we took ordinary Fourier transform (on the group $\mathbb{R}^{2}$ ) with respect to the second and third entry. Now by the substitutions $v=-(1 / 2) \lambda(y+p), u=p-y$ we find

$$
\begin{aligned}
\left\|\mathcal{F}_{H_{3}} W\right\|_{\mathbb{L}_{2}\left(T_{2}\left(\mathbb{L}_{2}\left(\mathbb{L}_{2}(\mathbb{R})\right)\right), \lambda \mathrm{d} \lambda\right)}^{2} & =\lambda^{-1} \int_{\mathbb{R}}\left\|K_{W}^{\lambda}\right\|_{2}^{2} \lambda \mathrm{d} \lambda \\
& =\int_{\mathbb{R}} \int_{\mathbb{R}} \int_{\mathbb{R}}\left|\mathcal{F}_{R^{2}}[W(u, \cdot, \cdot)](w, \lambda)\right|^{2} \mathrm{~d} u \mathrm{~d} v \mathrm{~d} \lambda=\|W\|_{\mathbb{L}_{2}\left(H_{3}\right)}^{2} .
\end{aligned}
$$

Consequently, one has the following formula for the inverse Fourier transform:

$$
\left(\mathcal{F}_{H_{3}}^{-1} \Phi\right)(p, q, s)=\int_{\mathbb{R}} \operatorname{trace}\left\{\Phi(\lambda) \mathcal{U}_{g}^{\lambda}\right\} \lambda \mathrm{d} \lambda,
$$

where we note that $\left(\mathcal{F}_{H_{3}}\right)^{*} \mathcal{F}_{H_{3}}=I$.

Now if we consider the reduced Weyl-Heisenberg group $H_{r}$ (obtained from $H_{3}$ by dividing out the center) then we have $\lambda=n \in \mathbb{Z}$

$$
\begin{aligned}
& \mathcal{F}_{H_{r}} W(n)=\int_{\mathbb{R}^{3}} W(p, q, s) \mathcal{U}_{-p,-q,-s}^{n} \phi(y) \mathrm{d} p \mathrm{~d} q \mathrm{~d} s \\
& \left(\mathcal{F}_{H_{r}}^{-1} \Phi\right)(p, q, s)=\sum_{n \in \mathbb{Z}} n \operatorname{trace}\left\{\Phi(n) \mathcal{U}_{g}^{n}\right\} .
\end{aligned}
$$

\section{B Differential geometry on (the domain of) Gabor-Transforms}

In this section we will first consider some basic differential geometry on the reduced Heisenberg group, which serves as a preliminary tool in the geometry driven diffusion in section 7 and reassignment on Gabor-transforms of section 6. We will mainly focus on the concept of horizontality, as this underlies all algorithms presented in this article.

As mentioned before the domain of a Gabor transform should not be considered as $\mathbb{R}^{2} \equiv H_{r} / \mathbb{T}$, while this implies that the whole fundamental non-commutative nature between position and frequency is lost. It should rather be considered as a principal fiber bundle. This is analogous to our previous work [12, ch:3.3] where the domain of orientation scores was considered as a principal fiber bundle. We will return to this strong analogy in section D. For now we restrict ourselves to a differential geometrical description of the domain of a Gabor transform.

Definition B.11. Let $M$ be a smooth manifold, $G$ be a Lie-group. A principal fiber bundle $P_{G}:=(P, M, \pi, R)$ above a manifold $M$ with structure group $G$ is a tuple $(P, M, \pi, R)$ such that $P$ is a smooth manifold, $\pi: P \rightarrow M$ is a smooth projection map with $\pi(P)=M, R$ a smooth right action $R_{g} p=p \cdot g, p \in P, g \in G$, such that $p \cdot(g h)=(p \cdot g) \cdot h$ and $\pi(p \cdot g)=\pi(p)$ for all $p \in P$, $g, h \in G$. Finally it should satisfy the "local triviality" condition, [41, p.346-347].

Within this article we shall restrict our selves to the special case where the principal fiber bundle is given by

$$
P_{T}:=\left(H_{r}, \mathbb{T}, \pi, R\right),
$$

where the fundamental projection $\pi: H_{r} \mapsto H_{r} / 0 \times\{0\} \times \mathbb{T}$ maps each element $g \in H_{r}$ on its associated coset $[g] \in H_{r} / 0 \times\{0\} \times \mathbb{T}$ is given by

$$
\pi(g)=g \mathbb{T}=\{g(0,0, t) \mid t \in \mathbb{R}\}=[g]=\left\{h \in H_{r} \mid h \sim g \Leftrightarrow g^{-1} h \in \mathbb{T}\right\} .
$$


So the structure group $\mathbb{T}$ acts from the right by means of $R_{h}=g h$ along the fibers, since obviously we have $\pi\left(R_{h} g\right)=\pi(g h)=\pi(g)$. Now by definition a Cartan-Ehresmann connection on such a principal fiber bundle is a Lie-algebra valued one-form $\omega: H_{r} \times T\left(H_{r}\right) \rightarrow T_{e}(\mathbb{T})$ defined on $P_{T}$ such that

$$
\begin{aligned}
& \omega(\mathrm{d} \mathcal{R}(A))=A \text { for all } A \in T_{e}(\mathbb{T}), \\
& \omega\left(\left(\mathcal{R}_{h}\right)_{*} \mathcal{A}\right)=\operatorname{Ad}\left(h^{-1}\right) \omega(\mathcal{A}) \text { for all vector fields } \mathcal{A} \text { on } H_{r} .
\end{aligned}
$$

In particular if we restrict ourselves to left-invariant vector fields we can take the so-called MaurerCartan form on $P_{\mathbb{T}}$ which is a $T_{e}(T)$ valued one-form on $P_{\mathbb{T}}$ defined on vectors $X_{g} \in T_{g}(P), g \in H_{r}$ and is given by

$$
\omega_{g}\left(X_{g}\right)=\left(\left.A^{3} \otimes \mathrm{d} \mathcal{A}^{3}\right|_{g}\right)\left(X_{g}\right)=\left\langle\left.\mathrm{d} \mathcal{A}^{3}\right|_{g}, X_{g}\right\rangle A_{3},
$$

so with respect to the fixed coordinate system $p, q, s$ we get

$$
\left\langle\mathrm{d} s+\frac{1}{2}(p \mathrm{~d} q-q \mathrm{~d} p), X_{g}\right\rangle \partial_{s},
$$

where we apply parallel-transport of tangent vectors over the commutative subgroups $\left(\mathbb{R}^{2}, 1\right)$ and $(0, \mathbb{T})$ to relate for example the tangent vector $\left.\partial_{p}\right|_{g}$ to $\partial_{p} \in T_{e}\left(H_{r}\right)$.

Now the horizontal part of the tangent space $T\left(H_{r}\right)$ is spanned by

$$
\operatorname{Ker}\{\omega\}=\operatorname{span}\left\{\mathcal{A}_{1}, \mathcal{A}_{2}\right\}=\operatorname{span}\left\{\partial_{p}+\frac{q}{2} \partial_{s}, \partial_{q}-\frac{p}{2} \partial_{s}\right\} .
$$

Definition B.12. A smooth curve $t \mapsto \gamma(t)$ in $H_{r}$ is called horizontal if all its tangent vectors lay in the horizontal part of each tangent space, i.e.

$$
\forall_{t>0} \omega_{\gamma(t)}(\dot{\gamma}(t))=0 \Leftrightarrow \forall_{t>0}\left\langle\left.\mathrm{~d} \mathcal{A}^{3}\right|_{\gamma(t)}, \dot{\gamma}(t)\right\rangle=0 .
$$

Lemma B.13. A smooth curve $t \mapsto \gamma(t)=(p(t), q(t), s(t))$ in $H_{r}$ is horizontal if and only if

$$
s(t)=s(0)+\frac{1}{2} \int_{0}^{t} q(\tau) p^{\prime}(\tau)-p(\tau) q^{\prime}(\tau) \mathrm{d} \tau,
$$

which has the following geometrical interpretation $s(t)$ is the net ${ }^{9}$ surface area enclosed by the planar curve $\tau \mapsto \tau(p(t), q(t)), \tau \in[0,1]$ and the planar curve $\tau \mapsto(p(\tau t), q(\tau t))-\left(p_{0}, q_{0}\right)$, $\tau \in[0,1]$

Proof. Let $t \mapsto \gamma(t)=(p(t), q(t), s(t))$ be a horizontal curve in $H_{3}$ then by definition

$$
\begin{aligned}
& \forall_{t>0}\left\langle\left.\mathrm{~d} \mathcal{A}^{3}\right|_{\gamma(t)}, \dot{\gamma}(t)\right\rangle=\left\langle\mathrm{d} s+\frac{1}{2}\left(p \mathrm{~d} q-\left.q \mathrm{~d} p\right|_{\gamma(t)}, \dot{\gamma}(t)\right\rangle=0\right. \\
& \Rightarrow s^{\prime}(t)=\frac{1}{2}\left(q(t) p^{\prime}(t)-p(t) q^{\prime}(t)\right),
\end{aligned}
$$

so that the first result follows by integration.

For the second part suppose that the closed concatenation-curve $K$ of the curve $K_{1}$ given by $\tau \mapsto(p(\tau t), q(\tau t)), \tau \in[0,1]$, followed by the curve $K_{2}$ given by $\tau \mapsto\left(p_{0}, q_{0}\right)+(2-\tau)((p(t)-$ $\left.p_{0}\right), \tau\left(q(t)-q_{0}\right), \tau \in[1,2]$ defines a negatively oriented closed surface $S$. Then by the Stokes Theorem in $3 D$ :

$$
\oint_{K=\partial S} \mathbf{F} \cdot \mathbf{t d} s=\int_{S} \operatorname{rot} \mathbf{F} \cdot \mathbf{n} \mathrm{d} \sigma,
$$

\footnotetext{
${ }^{9}$ positively oriented closed surfaces may cancel against negatively oriented subsurfaces if there are crossings of the curves $K_{1}$ and $K_{2}$, in which case to neighboring enclosed surfaces (by a simply connected subcurve constructed from a part of $K_{1}$ and a part of $K_{2}$ ) have opposite orientation.
} 
where $\mathbf{F}=(q,-p, 0)^{T}$ so that $\operatorname{rot} \mathbf{F}=(0,0,2)$ and $\mathbf{n}=(0,0,-1)$ so that

$$
\int_{K_{1}} \mathbf{F} \cdot \mathbf{t} \mathrm{d} s=\int_{0}^{t} q(\tau) p^{\prime}(\tau)-p(\tau) q^{\prime}(\tau) \mathrm{d} \tau+\int_{K_{2}} 0 \mathrm{~d} t=2 \int_{S} \mathrm{~d} \sigma=2 \mu(S) .
$$

Alternatively, one may consider the Stokes theorem by symplectic manifolds, i.e.

$$
\int_{\partial S} F=\int_{S} \mathrm{~d} F
$$

where $\mathrm{d}$ denotes the exterior derivative of the volume form $F$. In this we set $F=q \mathrm{~d} p-p \mathrm{~d} q$, so that

$$
\mathrm{d} F=\mathrm{d}(q \mathrm{~d} p-p \mathrm{~d} q)=\mathrm{d} q \wedge \mathrm{d} p-\mathrm{d} p \wedge \mathrm{d} q=2(\mathrm{~d} q \wedge \mathrm{d} p),
$$

so that $\int_{\partial S} q \mathrm{~d} p-p \mathrm{~d} q=2 \int_{S} \mathrm{~d} \sigma=2 \mu(S)$.

Now we follow the general construction in [39] and consider the associated vector bundle $P_{\mathbf{T}} \times \widetilde{\mathrm{Ad}} T_{e}\left(H_{r}\right)$ using the adjoint representation $\widetilde{A d}$ on the left-invariant vector fields given by

$$
\widetilde{\operatorname{Ad}}_{g} \mathcal{A}=\left(L_{h^{-1}} R_{h}\right)_{*} \mathcal{A}, \quad \mathcal{A} \in \mathcal{L}\left(H_{r}\right) .
$$

Intuitively speaking, one needs the representation $\widetilde{\mathrm{Ad}}$ to locate points on each fiber. Now the associated connection form (for details see [39], [31]) on the vector bundle is given by

$$
\begin{aligned}
\tilde{\omega} & =\sum_{j=1}^{3} \widetilde{\operatorname{Ad}}{ }_{*}\left(\mathcal{A}^{j}\right) \otimes \mathrm{d} \mathcal{A}^{j}=\sum_{j=1}^{3} \widetilde{\operatorname{ad}}\left(\mathcal{A}^{j}\right) \otimes \mathrm{d} \mathcal{A}^{j}=\sum_{j=1}^{3}\left[\cdot, \mathcal{A}^{j}\right] \otimes \mathrm{d} \mathcal{A}^{j}=\sum_{i, j, k=1}^{3} c_{i j}^{k} \mathcal{A}_{k} \otimes \mathrm{d} \mathcal{A}^{i} \otimes \mathrm{d} \mathcal{A}^{j} \\
& =-\partial_{s} \otimes \mathrm{d} \mathcal{A}_{1} \otimes \mathrm{d} \mathcal{A}_{2}+\partial_{s} \otimes \mathrm{d} \mathcal{A}_{2} \otimes \mathrm{d} \mathcal{A}_{1}=\partial_{s} \otimes \frac{1}{2} \mathrm{~d} p \wedge \mathrm{d} q,
\end{aligned}
$$

where $c_{i j}^{k}$ denote the structure constants of the Lie-algebra of $H_{r}$.

The Christoffel symbols of the Cartan connection

$$
D:=\mathrm{d}+\tilde{\omega}
$$

on the associated vector bundle $H_{r} \times \widetilde{\mathrm{Ad}} \mathcal{L}\left(H_{r}\right)$ are anti-symmetric and given by $\Gamma_{i j}^{k}=\tilde{\omega}\left(\mathcal{A}^{k}, \mathcal{A}_{j}, \mathcal{A}_{i}\right)=$ $-\left\langle\mathrm{d} \mathcal{A}^{k}, \widetilde{a d}\left(\mathcal{A}_{j}\right) \mathcal{A}_{i}\right\rangle=-c_{i j}^{k}$ and if $\left\{\mu_{k}\right\}_{k=1}^{3}$ are the sections in $E:=\left(H_{r}, T\left(H_{r}\right)\right)$ aligned with the left invariant vector fields $\left\{\mathcal{A}_{k}\right\}_{k=1}^{3}$ on $H_{r}$ the connection $D$ (B.68) in components reads

$$
(D \mu) X(t):=D_{X(t)} \mu(c(t))=\dot{a}^{k}(c(t)) \mu_{k}(c(t))+\dot{c}^{i} a^{k}(c(t)) \Gamma_{i k}^{j}(c(t)) \mu_{j}(c(t)),
$$

for all sections $\mu(c(t))=a^{k}(c(t)) \mu_{k}(c(t))$ and where $D_{\mathcal{A}_{i}} \mu_{j}=\Gamma_{i j}^{k} \mu_{k}$. For details see [31]p.108.

As the Christoffel symbols are anti-symmetric the auto-parallels (i.e. curves in $P$ which are co-variantly constant) satisfy the following equations

$$
\frac{d}{d t}\left\langle\mathrm{~d} \mathcal{A}^{k}, c^{\prime}(t)\right\rangle=-\Gamma_{i j}^{k}\left\langle\mathrm{~d} \mathcal{A}^{i}, c^{\prime}(t)\right\rangle\left\langle\mathrm{d} \mathcal{A}^{j}, c^{\prime}(s)\right\rangle=0
$$

and thereby we have that

$$
\left\langle\mathrm{d} \mathcal{A}^{k}, c^{\prime}(t)\right\rangle=\left\langle\mathrm{d} \mathcal{A}^{k}, c^{\prime}(0)\right\rangle=\text { constant }=c^{k} \text { for } k=1, \ldots, n,
$$

so $c^{\prime}(t)=\left.\sum_{k=1}^{3} c^{k} \mathcal{A}_{k}\right|_{c(t)}$, for some constants $c_{k} \in \mathbb{R}$. Now these curves exactly coincide with the exponential curves $c(t)=c(0) \exp \left(\left.t \sum_{k=1}^{3} c^{k} \mathcal{A}_{k}\right|_{c(0)}\right)$ within $H_{r}$, which directly follow from (2.12):

$$
c(t)=\left(p_{0}+a_{1} t, q_{0}+a_{2} t, s_{0}+t\left(a_{3}+\frac{1}{2}\left(q_{0} a_{1}-p_{0} a_{2}\right)\right)\right) .
$$


Now the Theorems [12, thm.3.8, thm 3.9] in our previous work on invertible orientation scores can be straight-forwardly generalized to Gabor transforms. Consequently, we can replace the leftinvariant vector fields in our non-linear, adaptive convection and diffusion schemes by covariant derivatives and both convection and diffusion locally takes place along the covariantly constant (or auto-parallel) curves which coincide with exponential curves (B.71).

Furthermore, in our previous work [12, thm 3.13] we have explained there are two equivalent ways the consider the domain of an orientation score. Either one considers the domain of an orientation score as a principal fiber bundle $P_{Y}$, which is the natural equivalent to considering the domain of a Gabor transform as $P_{\mathbb{T}}$, or one considers the domain of an orientation score as a contact manifold $(S E(2),-\sin \theta \mathrm{d} x+\cos \theta \mathrm{d} y)$. To keep track of the domain itself rather than its tangent bundle, the viewpoint via principal fiber bundles is preferable. For optimization of Lagrangians, however, one needs the (co)-tangent bundle and here it is rather the viewpoint of contact manifolds keeps things simple. Since we are interested in optimizing Lagrangians later on in Section D we briefly mention the contact manifold view-point on the domain of orientation scores.

To this end we note that the well-known Maurer-Cartan structural equation, [6], reads

$$
\mathrm{d}\left(\mathrm{d} \mathcal{A}^{k}\right)=-\frac{1}{2} c_{i j}^{k} \mathrm{~d} \mathcal{A}^{\mathrm{i}} \wedge \mathcal{A}^{j}
$$

so more explicitly, in our case of $H_{r}$, we have

$$
\mathrm{dd} s=\mathrm{d} q \wedge \mathrm{d} p, \quad \operatorname{dd} p=0, \quad \operatorname{dd} q=0
$$

and in particular along the horizontal part of the tangent space we have

$$
\mathrm{dd} s=\frac{1}{2} \mathrm{~d}(q \mathrm{~d} p-p \mathrm{~d} q)=\frac{1}{2} \mathrm{~d} q \wedge \mathrm{d} p-\frac{1}{2} \mathrm{~d} p \wedge \mathrm{d} q=\mathrm{d} q \wedge \mathrm{d} p .
$$

Definition B.14. A contact manifold $(M, I)$ is a smooth manifold $M$ of dimension $2 n+1, n \in \mathbb{N}$, with a distinguished line sub-bundle $I \subset T^{*}(M)$ of the co-tangent bundle which is non-degenerate in the sense that for any local 1-form $\theta$ generating $I$, we have

$$
\theta \wedge(\mathrm{d} \theta)^{n} \neq 0 .
$$

So we conclude that the Weyl-Heisenberg group $\left(H_{r}, \mathrm{~d} \mathcal{A}^{3}=\mathrm{d} s+\frac{1}{2}(q \mathrm{~d} p-p \mathrm{~d} q)\right)$ is a contact manifold of dimension 3 , since by the preceding we have $\mathrm{d}\left(\mathrm{d} \mathcal{A}^{3}\right)=\mathrm{dd} s-\frac{1}{2}(\mathrm{~d} p \wedge \mathrm{d} q-\mathrm{d} q \wedge \mathrm{d} p)=$ $2 \mathrm{~d} q \wedge \mathrm{d} p$ and therefore

$$
\mathrm{d} \mathcal{A}^{3} \wedge \mathrm{d}\left(\mathrm{d} \mathcal{A}^{3}\right)=\left(\mathrm{d} s+\frac{1}{2}(q \mathrm{~d} p-p \mathrm{~d} q)\right) \wedge(2 \mathrm{~d} q \wedge \mathrm{d} p)=2 \mathrm{~d} q \wedge \mathrm{d} p \wedge \mathrm{d} s \neq 0 .
$$

Remark B.15. Here we recall Remark 2.3, $\operatorname{so}^{10} \mathrm{~d}\left(\mathrm{~d} \mathcal{A}^{3}\right) \neq " \mathrm{~d}^{2} \mathcal{A}^{3} "$, since the right $\mathrm{d}$ with the round brackets is not an exterior derivative.

\section{$\mathrm{C}$ Contact geometry and geodesics in the Gabor domain}

If we would like to impose a left invariant first fundamental tensor $\mathcal{G}: H_{r} \times T\left(H_{r}\right) \times T\left(H_{r}\right) \rightarrow \mathbb{C}$ (or "metric") on $H_{r}$, which means that

$$
\forall_{h, g \in H_{r}} \forall_{X, Y \in \chi\left(H_{r}\right)} \mathcal{G}_{h}\left(X_{h}, Y_{h}\right)=\mathcal{G}_{g h}\left(\left(L_{g}\right)_{*} X_{h},\left(L_{g}\right)_{*} Y_{h}\right),
$$

where $\chi\left(H_{r}\right)$ denotes the vector space of all vector fields on $H_{r}$, then it is clear form this definition that such a first fundamental tensor must be of the form

$$
\mathcal{G}=\sum_{i, j=1}^{3} g_{i j} \mathrm{~d} \mathcal{A}^{i} \otimes \mathrm{d} \mathcal{A}^{j},
$$

\footnotetext{
${ }^{10}$ In fact, here the right-hand side does not make sense, since $\mathcal{A}_{3}$ is a vector not a co-vector.
} 
where $g_{i j}$ are constants. For formal proof see [31, p.266]. Moreover, the first fundamental form should be diagonal, with similar arguments as in [12, Thm 3.1].

However, as noted in the introduction and section 2 the domain of a Gabor transform should neither be considered as $H_{3}$ nor as $H_{3} / \mathbb{T}$ but rather as the principal fiber bundle $P_{\mathbb{T}}=\left(H_{3}, \mathbb{T}, \pi, R\right)$ on which we impose the following first fundamental form:

$$
\mathcal{G}_{\beta}=g_{i j} \mathrm{~d} \mathcal{A}^{i} \otimes \mathrm{d} \mathcal{A}^{j}=\beta^{4} \mathrm{~d} \mathcal{A}^{1} \otimes \mathrm{d} \mathcal{A}^{1}+\mathrm{d} \mathcal{A}^{2} \otimes \mathrm{d} \mathcal{A}^{2},
$$

which is bijectively related to the linear operator $G: H \rightarrow H^{\prime}$ which maps $\mathcal{A}_{1}$ to $\beta^{4} \mathrm{~d} \mathcal{A}^{1}$ and $\mathcal{A}_{2}$ to $\mathrm{d} \mathcal{A}^{2}$. The inverse operator of $G$ is bijectively related to

$$
\mathcal{G}_{\beta}^{-1}=g^{i j} \mathcal{A}_{i} \otimes \mathcal{A}_{j}=\beta^{-4} \mathcal{A}_{1} \otimes \mathcal{A}_{1}+\mathcal{A}_{2} \otimes \mathcal{A}_{2}
$$

Here the fundamental positive parameter $\beta^{-1}$ has physical dimension length, so that this first fundamental form is consistent with respect to physical dimensions. Intuitively, the parameter $\beta$ sets a global balance between changes in frequency space and changes in position space.

Now the Cauchy-Riemann relations (2.32) that hold between local phase and local amplitude are for $\beta^{-1}=a$ simply written as

$$
\mathcal{G}_{\beta=\frac{1}{a}}^{-1}(\mathrm{~d} \log |U|, \mathrm{d} \Omega)=0,
$$

where the left-invariant gradient equals $\mathrm{d} \Omega=\sum_{i=1}^{3} \mathcal{A}_{i} \Omega \mathrm{d} \mathcal{A}^{i}$ and where $U=\mathcal{W}_{\psi_{a}} f$ with $\psi_{a}(\xi)=$ $e^{-a^{-2} \xi^{2} n \pi}, a>0$. which gives us a geometric understanding of this fundamental form. The normal vector $\left.\mathrm{d} \Omega\right|_{g_{0}}$ to the surface $\left\{(p, q, s) \in H_{r} \mid \Omega(p, q, s)=\right.$ constant $\left.=\Omega\left(g_{0}\right)\right\}$ is $\mathcal{G}_{\beta}$-orthogonal to the normal vector $\mathrm{d} \mid U \|_{g_{0}}$ to the surfaces $\left\{(p, q, s) \in H_{r}|| U \mid(p, q, s)=\right.$ constant $\left.=|U|\left(g_{0}\right)\right\}$.

\section{C.1 Geodesics in the Heisenberg group}

In order to find the geodesics we apply a coordinates transform

$$
\left\{\begin{array} { l } 
{ p = x } \\
{ q = \beta \theta } \\
{ s = \beta ( y - \frac { x \theta } { 2 } ) }
\end{array} \Leftrightarrow \left\{\begin{array}{l}
x=p \\
y=\beta^{-1}\left(s+\frac{p q}{2}\right) \\
\theta=\beta^{-1} q .
\end{array}\right.\right.
$$

Note that, for $\beta=1$ this coincides with the fundamental coordinate transform which relates the coordinates of the first to the coordinates of the second kind, recall (2.13) and (2.14). Furthermore we note that the coordinate transform is consistent with the physical dimensions, as the physical dimension of $\beta^{-1}$ equals length. Note that

$$
\partial_{p}=\partial_{x}+\frac{\theta}{2} \partial_{y}, \quad \partial_{q}=\frac{x}{2 \beta} \partial_{y}+\beta^{-1} \partial_{\theta}, \quad \partial_{s}=\beta^{-1} \partial_{y},
$$

and as a result the left-invariant vector fields are given by

$$
\left\{\mathcal{A}_{1}, \mathcal{A}_{2}, \mathcal{A}_{3}\right\}=\left\{\partial_{p}+\frac{q}{2} \partial_{s}, \partial_{q}-\frac{p}{2} \partial_{s}, \partial_{s}\right\}=\left\{\partial_{x}+\theta \partial_{y}, \beta^{-1} \partial_{\theta}, \beta^{-1} \partial_{y}\right\}
$$

which (in case the periodicity in $\theta$-direction is dropped, i.e. one considers $H_{3}$ rather than $H_{3} /\{0\} \times$ $\beta^{-1} \mathbb{Z} \times\{0\}$ where we recall $\left.q=\beta \theta\right)$ are the generators of the Heisenberg group of velocities (normalized in $x$ direction).

The main reason for this coordinate transform is that the Lie-algebra of the Heisenberg group $H_{3}$ is isomorphic to the Lie-algebra of the contraction group $\lim _{t \downarrow 0}(S E(2))_{t}=(S E(2))_{0}$ that we derived from the Euclidean motion group $S E(2)$ in [11] (part I). The group products on the intermediate groups $(S E(2))_{t \in(0,1]}$ are given by

$$
(x, y, \theta) \cdot{ }_{t}\left(x^{\prime}, y^{\prime}, \theta^{\prime}\right)=\left(x+\cos (\theta t) x^{\prime}-t \sin (\theta t) y^{\prime}, y+\frac{\sin (\theta t)}{t} x^{\prime}+\cos (\theta t) y^{\prime}, \theta+\theta^{\prime}\right) .
$$


So the group $(S E(2))_{t}$ is isomorphic to the matrix group

$$
\left\{\left(\begin{array}{ccc}
\cos (\theta t) & -t \sin (\theta t) & x \\
\frac{\sin (\theta t)}{t} & \cos (\theta t) & y \\
0 & 0 & 1
\end{array}\right) \mid(x, y) \in \mathbb{R}^{2}, \theta \in[0,2 \pi)\right\} .
$$

The left-invariant vector fields of the intermediate groups $(S E(2))_{t \in(0,1]}$ are given by

$$
\mathcal{A}_{1}^{t}=\partial_{\theta}, \quad \mathcal{A}_{2}^{t}=\cos (\theta t) \partial_{x}+\frac{\sin (\theta t)}{t} \partial_{y}, \quad \mathcal{A}_{3}^{t}=-t \sin (\theta t) \partial_{x}+\cos (\theta t) \partial_{y} .
$$

Now the horizontal part of a tangent space $T_{g}\left((S E(2))_{t}\right), t \in[0,1]$ is given by

$$
H_{g}^{t}=\operatorname{span}\left\{\mathcal{A}_{1}^{t}, \mathcal{A}_{2}^{t}\right\}
$$

so for example at $t=1$ we have $(S E(2))_{t=1}=S E(2)$ and $H_{g}^{t}=\operatorname{span}\left\{\partial_{\theta}, \partial_{\xi}\right\}, \partial_{\xi}=\cos \theta \partial_{x}+$ $\sin \theta \partial_{y}$, which were the important horizontal directions in our previous work [12] on diffusion on invertible orientation scores (which are equivalent to Gabor transforms in the sense that the representation $(2.15)$ on $\mathbb{L}_{2}(\mathbb{R})$ is replaced by the left-regular representation of $S E(2)$ on $\mathbb{L}_{2}\left(\mathbb{R}^{2}\right)$ ).

However at $t=0$ we have $(S E(2))_{t=0}=H_{r}$ and

$$
H_{g}^{0}=\operatorname{span}\left\{\partial_{x}+\theta \partial_{y}, \partial_{\theta}\right\} \equiv \operatorname{span}\left\{\partial_{p}+\frac{q}{2} \partial_{s}, \partial_{q}-\frac{p}{2} \partial_{s}\right\}
$$

Here we note that due to different ordering conventions of our previous work in $S E(2)$ and the common convention on $H_{3}$ we use here, we have that $\mathcal{A}_{1}^{t \downarrow 0}=\beta \mathcal{A}_{2}=\partial_{\theta}$ and $\mathcal{A}_{2}^{t \downarrow 0}=\mathcal{A}_{1}=\partial_{x}+\theta \partial_{y}$ and as a result $\mathrm{d} \mathcal{A}_{1}^{t \downarrow 0}=\beta^{-1} \mathrm{~d} \mathcal{A}^{2}$ and $\mathrm{d} \mathcal{A}_{2}^{t \downarrow 0}=\mathrm{d} \mathcal{A}^{1}$.

So we see that $\left((S E(2))_{t}, \mathrm{~d} \mathcal{A}_{3}^{t}\right)$ is a contact manifold with horizontal tangent spaces

$$
\left\{v \in T\left((S E(2))_{t}\right) \mid \mathrm{d} \mathcal{A}_{3}^{t} v=-\frac{\sin (\theta t)}{t}\langle\mathrm{~d} x, v\rangle+\cos (\theta t)\langle\mathrm{d} y, v\rangle=0\right\}
$$

so along horizontal curves in $(S E(2))_{t}$ one has the relation

$$
\frac{d y}{d x}=\left(\frac{\tan \theta t}{\theta t}\right) \theta
$$

and taking the limit $t \rightarrow 0$ we arrive at the relation along horizontal curves in $H_{3}$ :

$$
\frac{d y}{d x}=\theta
$$

The preceding shows us that just like we were able to relate geodesics in $S E(2)$ to elastica curves, [12] we can relate geodesics in $H_{3}$ to B-splines, [13],[15], simply by taking the square of the Lagrangian associated to the left-invariant metric on the underlying fiber-bundle. ${ }^{11}$

Consider the following distance on the contact manifold $(S E(2))_{t}, t \in[0,1]$ :

$$
d(a, b)=\inf _{\Gamma} \int_{0}^{1}\left\|\gamma^{\prime}(r)\right\|_{t, \beta} \mathrm{d} r \text { under the condition } \Gamma \text { horizontal and } \gamma(0)=a, \gamma(1)=b,
$$

\footnotetext{
${ }^{11}$ On Riemannian manifolds, it does not yield different minimal curves if one takes the square of the Lagrangian associated to the metric-connection, if one uses arc-length parametrization. According to [38]p.490 on contact manifolds (sub-Riemannian manifolds), such as $(S E(2))_{t}$, it does not make a difference either if all curves are defined in the same interval. However, if one insists on a different parametrization than arclength in the sub-Riemannian manifold differences may appear (while insisting on certain identities). For example in $S E(2)=\mathbb{R}^{2} \rtimes \mathbb{T}$ one needs arc-length $s>0$ parametrization $s \mapsto(x(s), y(s), \arg (\dot{x}(s)+i \dot{y}(s)))$, where $s>0$ is arclength of the projected curve $\mathbf{x}=(x, y)$ on $\mathbb{R}^{2}$, in order to ensure $\kappa=\frac{d \theta}{d s}$ along a horizontal curve, and in $H(3)$ we need parametrization by $x$, $x \mapsto(x, y(x), \theta(x))$ in order to ensure that $\theta=\frac{d y}{d x}$. For a clear comparison between optimization of homogenous Lagrangians and non-homogenous Lagrangians see [40].
} 
where we note that $\Gamma$ is horizontal iff $\left\langle\left.\mathrm{d} \mathcal{A}_{3}^{t}\right|_{\gamma(r)}, \gamma^{\prime}(r)\right\rangle=0$ for all $r \in[0,1]$ and where

$$
\begin{aligned}
\left\|\gamma^{\prime}(r)\right\|_{t, \beta} & =\sqrt{\beta^{2}\left|\left\langle\mathrm{~d} \mathcal{A}_{2}^{t}, \dot{\gamma}(r)\right\rangle\right|+\left|\left\langle\mathrm{d} \mathcal{A}_{1}^{t}, \dot{\gamma}(r)\right\rangle\right|} \\
& =\sqrt{\beta^{2}|\langle\cos (\theta t) \mathrm{d} x+t \sin (\theta t) \mathrm{d} y, \dot{\gamma}(r)\rangle|^{2}+|\langle\mathrm{d} \theta, \dot{\gamma}(r)\rangle|^{2}} \mathrm{~d} r
\end{aligned}
$$

So for respectively $t=1$ and $t=0$ we get

$$
\begin{aligned}
& \left\|\gamma^{\prime}(s)\right\|_{1, \beta}=\sqrt{|\dot{\theta}(s)|^{2}+\beta^{2}}=\sqrt{\kappa(s)^{2}+\beta^{2}} \text { and } \\
& \left\|\gamma^{\prime}(x)\right\|_{0, \beta}=\sqrt{\left(\theta^{\prime}(x)\right)^{2}+\beta^{2}}=\sqrt{\left(y^{\prime \prime}(x)\right)^{2}+\beta^{2}},
\end{aligned}
$$

where $s$ is the arclength of the spatial curve $\mathbb{P}_{\mathbb{R}^{2}} \gamma$ and where $x$ represents the $x$-coordinate of the spatial curve $\mathbb{P}_{\mathbb{R}^{2}} \gamma$.

The case $t=1$ is already solved in [12]. For $t=0$ we proceed analogously.

For $t=0$ the energy is given by

$$
\mathcal{E}(\gamma)=\int_{0}^{x_{\max }} \sqrt{\left(y^{\prime \prime}(x)\right)^{2}+\beta^{2}} \mathrm{~d} x
$$

and we must study how this energy changes under horizontal curve deviations. Again there is no need to consider all 3-types of horizontal curve pertubations and we can restrict our-selves to a single horizontal curve pertubation:

$$
\gamma=(x, y, \theta) \mapsto \gamma_{\delta}^{\epsilon}=\left(x, y+\epsilon \delta, \theta+\epsilon \delta^{\prime}\right)
$$

and note $\gamma$ horizontal iff $\gamma_{\delta}^{\epsilon}$ horizontal. Now

$$
\lim _{\epsilon \rightarrow 0} \frac{\mathcal{E}\left(\gamma_{\delta}^{\epsilon}\right)-\mathcal{E}(\gamma)}{\epsilon}=0 \Leftrightarrow \lim _{\epsilon \rightarrow 0} \int_{0}^{x_{m} a x} \sqrt{\left(y^{\prime \prime}(x)\right)^{2}+\beta^{2}}\left(\sqrt{1+\frac{2 \epsilon \delta^{\prime \prime}(x) y^{\prime \prime}(x)}{\left(y^{\prime \prime}(x)\right) 2+\beta^{2}}}-1\right) \mathrm{d} x=0
$$

Now use the approximation $\sqrt{1+x} \approx 1+\frac{1}{2} x+O\left(x^{2}\right)$ so that we find

$$
\lim _{\epsilon \rightarrow 0} \frac{\mathcal{E}\left(\gamma_{\delta}^{\epsilon}\right)-\mathcal{E}(\gamma)}{\epsilon}=0 \Leftrightarrow \int_{0}^{x_{\max }} \frac{y^{\prime \prime}(x) \delta^{\prime \prime}(x)}{\sqrt{\left(y^{\prime \prime}(x)\right)^{2}+\beta^{2}}} \mathrm{~d} x=0 \text { for all } \delta \in \mathcal{D}\left(\left(0, x_{\max }\right)\right),
$$

so we find the following Euler-Lagrange equation

$$
\left(\frac{y^{\prime \prime}(x)}{\sqrt{\left(y^{\prime \prime}(x)\right)^{2}+\beta^{2}}}\right)^{\prime \prime}=0 \Leftrightarrow\left(\frac{v}{\sqrt{v^{2}+\beta^{2}}}\right)^{\prime \prime}=0
$$

with $v=y^{\prime \prime}$. So that we find by explicit differentiation:

$$
-3 \beta^{2} v\left(v^{\prime}\right)^{2}+\left(\beta^{2}+v^{2}\right) v^{\prime \prime}=0,
$$

the general solution of which is given by

$$
v(x)=y^{\prime \prime}(x)=\frac{c_{1} \beta\left(x+c_{2}\right)}{\sqrt{\beta^{-4}-c_{1}^{2}\left(x+c_{2}\right)^{2}}}
$$

Now integrating this equation yields

$$
\theta(x)=y^{\prime}(x)=-\sqrt{\frac{1}{\left(\beta c_{1}\right)^{2}-\left(\beta\left(x+c_{2}\right)\right)^{2}}}+\sqrt{\frac{1}{\left(\beta c_{1}\right)^{2}-\left(\beta\left(x+c_{2}\right)\right)^{2}}}+\theta_{0} .
$$

Another integration yields

$$
y(x)=y_{0}+\left(\sqrt{\frac{1}{\left(\beta c_{1}\right)^{2}}-\beta^{2}\left(c_{2}\right)^{2}}\right) x-\frac{\beta}{2}\left[\tilde{x} \sqrt{\frac{1}{\beta^{4} c_{1}^{2}}-\tilde{x}^{2}}+\frac{1}{\beta^{4} c_{1}^{2}} \arcsin \left(\tilde{x} \beta^{2}\left|c_{1}\right|\right)\right]_{\tilde{x}=x}^{\tilde{x}=x+c_{2}} .
$$


Now we may use left-invariance and assume that $a=e=(0,0,0) \theta_{0}=x_{0}=y_{0}=0$. Then $c_{1}$ and $c_{2}$ follow by

$$
b=\gamma\left(x_{\max }\right)=\left(x_{\max }, y\left(x_{\max }\right), \theta\left(x_{\max }\right)\right), \text { with } b=\left(x_{1}, y_{1}, \theta_{1}\right) .
$$

This system can not be solved analytically. However, for $0<\beta<<1$ we can use an asymptotic expansion $\beta \mapsto \frac{1}{2 \beta^{3}\left(c_{1}\right)^{2}} \arcsin \left(x \beta^{2}\left|c_{1}\right|\right) \approx \frac{x \beta^{2}\left|c_{1}\right|}{2 \beta^{3} c_{1}^{2}}=\frac{x}{2 \beta\left|c_{1}\right|}$. Using this expansion and $a=e$ we find

$$
\begin{aligned}
& \theta_{1}=-\sqrt{\frac{1}{\left(\beta c_{1}\right)^{2}}-\beta^{2}\left(x_{1}+c_{2}\right)^{2}}+\sqrt{\frac{1}{\left(\beta c_{1}\right)^{2}}-\beta^{2}\left(c_{2}\right)^{2}} \\
& y_{1} \approx-\frac{x_{1}}{\beta\left|c_{1}\right|}\left(\frac{\sqrt{1-\beta^{4} c_{1}^{2} x_{1}^{2}}+1}{2}-\sqrt{1-\left(\beta c_{2}\right)^{2}\left(\beta c_{1}\right)^{2}}\right),
\end{aligned}
$$

which can be solved analytically.

An alternative approach to solve the Euler-Lagrange equation (C.79) follows by using the fact that $\theta(x)=y^{\prime}(x)$ along any horizontal curve in $H_{3}$. Since then we see that

$$
\left(\frac{\theta^{\prime}}{\sqrt{\left(\theta^{\prime}\right)^{2}+\beta^{2}}}\right)^{\prime}=\frac{\beta^{-1} \theta^{\prime \prime}}{\left(\beta^{-2}\left(\theta^{\prime}\right)^{2}+1\right)^{\frac{3}{2}}}=c \beta
$$

for some constant $c \in \mathbb{R}^{+}$. Now apply the inverse coordinate trafo and we get

$$
\left(\left(\beta^{-2} \frac{d q}{d p}\right)^{2}+1\right)^{-\frac{3}{2}} \frac{\beta^{-2} d^{2} q}{d p^{2}}=c \beta
$$

Now the left-hand side equals the Euclidean curvature of the graph $p \mapsto \beta^{-2} q(p)$ and the righthand side is constant. Consequently, the geodesics have to lie on a cilinder

$$
(p-a)^{2}+\left(\beta^{-2} q-b\right)^{2}=\frac{1}{c^{2} \beta^{2}},
$$

for some constants $a, b, c$. As a result we may parameterize the solutions as follows, where by left-invariance we (may) assume $p(0)=q(0)=s(0)=0$ :

$$
\begin{aligned}
& p(\tau)=\frac{1}{c \beta}(\sin (\omega \tau+\phi)-\sin \phi), \\
& q(\tau)=\frac{\beta}{c}(\cos (\omega \tau+\phi)-\cos \phi), \\
& s(\tau)=\frac{1}{2} \int_{0}^{\tau} q^{\prime}(\tilde{\tau}) p(\tilde{\tau})-p(\tilde{\tau}) q^{\prime}(\tilde{\tau}) \mathrm{d} \tilde{\tau}=\frac{1}{2} \frac{\omega}{c^{2}} \tau-\frac{1}{2 c^{2}} \sin (\omega \tau),
\end{aligned}
$$

where $\tau \in\left[0, \tau_{\max }\right)$. For $\beta=1$ this formula coincides with a well-known parametrization of geodesics in $H_{r}$, [38]. We have set $a=(c \beta)^{-1} \sin \phi, b=(c \beta)^{-1} \cos \phi$, so that $\left(p_{1}-\frac{1}{c \beta} \sin \phi\right)^{2}+$ $\left(\beta^{-2} q_{1}-\frac{1}{c \beta} \cos \phi\right)^{2}=(c \beta)^{-2}$. This yields

$$
c=\frac{1}{\beta} \frac{2\left(p_{1} \sin \phi+\beta^{-2} q_{1} \cos \phi\right)}{\left(p_{1}\right)^{2}+\beta^{-4} q_{1}^{2}} .
$$

Now the three remaining parameters $\tau_{\max }, \phi$ and $\omega$ are determined by

$$
p\left(\tau_{\max }\right)=p_{1}, q\left(\tau_{\max }\right)=q_{1} \text { and } s\left(\tau_{\max }\right)=s_{1} .
$$

\section{C.2 The quadratic Lagrangian on $H_{3}$}

If we take the square of the Lagrangian in the energy (with $\theta(x)=y^{\prime}(x)$ ) one looses the parametrization independency of the distance. However, one gets simpler solutions: namely B-splines. This is easily seen since if we set

$$
\mathcal{E}(\gamma)=\int_{0}^{x_{\max }}\left(\left(y^{\prime \prime}(x)\right)^{2}+\beta\right) \mathrm{d} x
$$


in stead of (C.78) the Euler-Lagrange equation simply becomes $y^{\prime \prime \prime \prime}(x)=0$, yielding a third order polynomial. So then we have

$$
\begin{aligned}
\tilde{d}_{t=0}(a, b) & =\inf _{\Gamma} \int_{0}^{x}\left\|\gamma^{\prime}(r)\right\|_{t=0, \beta}^{2} \mathrm{~d} r \text { under the condition } \Gamma \text { horizontal and } \gamma(0)=a, \gamma(x)=b, \\
& =\min \left\{\int_{0}^{x}\left(\left(y^{\prime \prime}(r)\right)^{2}+\beta^{2}\right) \mathrm{d} r \mid y(0)=y, y(0)=0, y^{\prime}(0)=0, y^{\prime}(0)=\theta, y^{\prime}(r)=\theta(r),\right\} \\
& =4 \frac{3 y^{2}+3 x y \theta+x^{2} \theta^{2}}{x^{3}}+\beta^{2} x
\end{aligned}
$$

with $a=(0,0,0), b=(x, y, \theta)$. The corresponding optimal curves are given by

$$
\begin{aligned}
y(x) & =x \theta_{0}+\frac{x^{3}}{x_{1}^{3}}\left(-2 y_{1}+x_{1}\left(\theta_{0}-\theta_{1}\right)\right)+\frac{x^{2}}{x_{1}^{2}}\left(3 y_{1}+x_{1}\left(\theta_{1}-2 \theta_{0}\right)\right) \\
& +\frac{\left(\kappa_{0}-\kappa_{1}\right)}{x_{1}^{3}}\left(x-x_{1}\right)^{2}\left(\frac{x_{1}}{2}-x\right) x^{2} \\
\theta(x) & =\theta_{0}+2 \frac{x}{x_{1}^{2}}\left(3 y_{1}+x_{1}\left(\theta_{1}-2 \theta_{0}\right)\right)-3 \frac{x^{2}}{x_{1}^{3}}\left(2 y_{1}+x_{1}\left(\theta_{1}-\theta_{0}\right)\right) \\
& +\frac{\left(\kappa_{0}-\kappa_{1}\right)}{x_{1}^{3}} x\left(x-x_{1}\right)\left(-3 x^{2}+3 x_{1} x-x_{1}^{2}\right)
\end{aligned}
$$

where $x \in\left[0, x_{1}\right]$ and $y(0)=0, \theta(0)=\theta_{0}$ and $y\left(x_{1}\right)=y_{1}, \theta\left(x_{1}\right)=-\theta_{1}$ and $\frac{d y}{d x}(0)=\theta_{0}$ and $\frac{d y}{d x}\left(x_{1}\right)=$ $-\theta_{1}$.

\section{Computations of the geodesics on the intermediate groups $(S E(2))_{t}$ between $H_{3}$ and $S E(2)$ by means of reduction on Pfaffian systems using Noether's theorem}

Here we generalize our results, [12] Appendix A for the Euclidean motion group case $t=1$, to the general case $t \in[0,1]$ in order to solve the problem (C.77) for all $t \in[0,1]$. We will investigate how the geodesics and the corresponding conservation-laws deform with $t>0$. This is useful as this allows us to relate our linear and non-linear left-invariant evolution equations on invertible orientation scores defined on $S E(2)$ (with the practical goal of enhancement/detection of elongated structures in 2D-images, [12], [18]), to the left-invariant evolutions on Gabor transforms defined on $H(3)$ (with the practical goal of enhancement of local frequencies in 1D-signals).

Consider the manifold $Q=(S E(2))_{t} \times \mathbb{R}^{+} \times \mathbb{R} \times \mathbb{R}$ with coordinates $\left(x, y, e^{i \theta}, \sigma, \kappa, r\right)$, where $\sigma=\left\|\mathbf{x}^{\prime}(r)\right\|$ so that $\mathrm{d} s=\sigma \mathrm{d} r$. On $Q$ we consider the Pfaffian equations

$$
\begin{aligned}
& \theta^{1}:=\mathrm{d} \theta-\kappa f_{t}(\theta) \sigma \mathrm{d} r=0, \\
& \theta^{2}:=\mathrm{d} \mathcal{A}_{2}^{t}-\frac{1}{\sqrt{f_{t}(\theta)}} \sigma \mathrm{d} r=\cos (\theta t) \mathrm{d} x+t \sin (\theta t) \mathrm{d} y-\frac{1}{\sqrt{f_{t}(\theta)}} \sigma \mathrm{d} r=0, \quad \sigma>0, \\
& \theta^{3}:=\mathrm{d} \mathcal{A}_{3}^{t}=-\frac{\sin (\theta t)}{t} \mathrm{~d} x+\cos (\theta t) \mathrm{d} y=0 .
\end{aligned}
$$

where $\sigma>0$ and $f_{t}(\theta):=\cos ^{2}(\theta t)+t^{-2} \sin ^{2}(\theta t) t \in(0,1]$ and $f_{0}(\theta)=\lim _{t \downarrow 0} f_{t}(\theta)=1+\theta^{2}$.

These Pfaffian equations uniquely determine the horizontal part $I(Q)$ of the dual tangent space $T^{*}(Q)$. To this end we recall that along horizontal curves $\gamma=(\mathbf{x}, \theta)$ we have $\left\langle\mathrm{d} \mathcal{A}_{3}^{t}, \dot{\gamma}\right\rangle=0$, or equivalently (C.76), from which we deduce that

$$
\begin{aligned}
& \kappa=\left\|\frac{d^{2} \mathbf{x}}{d s^{2}}\right\|=\frac{1}{\sqrt{1+\left(y^{\prime}(x)\right)^{2}}}\left\|\frac{d}{d x} \frac{1}{\sqrt{1+\left(y^{\prime}(x)\right)^{2}}}\left(1, y^{\prime}(x)\right)\right\|=\frac{\left|y^{\prime \prime}(x)\right|}{\left(1+\left(y^{\prime}\right)^{2}\right)^{\frac{3}{2}}} \Leftrightarrow \\
& \kappa=\frac{1}{\cos ^{2}(\theta t)+t^{-2} \sin ^{2}(\theta t)} \frac{\mathrm{d} \theta}{d s}=\frac{1(\theta)}{d s} .
\end{aligned}
$$

and furthermore we have by $\mathrm{d} s \equiv \mathrm{d} \mathcal{A}_{2}^{t}$ and

$$
\|\dot{x}(s)\|_{\mathbb{R}^{2}}=\left\|\left\langle d \mathcal{A}_{t}^{2}, \dot{\gamma}\right\rangle \mathcal{A}_{2}^{t}\right\|_{\mathbb{R}^{2}}=\sqrt{f_{t}(\theta)}\left|\left\langle\mathrm{d} \mathcal{A}_{t}^{2}, \dot{\gamma}\right\rangle\right|=1
$$


The objective is to minimize the parametrization independent energy (C.77), with an extra a priori cost-function $^{12} \tilde{C}:(S E(2))_{t} \rightarrow[1, \infty)$ on the group $(S E(2))_{t}$, in which we set $r=s$ and use the identities (D.85) and (D.86) which hold along any horizontal curve

$$
\begin{aligned}
\inf _{\gamma} \int_{0}^{1} \tilde{C}(\gamma(r))\left\|\gamma^{\prime}(r)\right\|_{t, \beta} \mathrm{d} r & =\int_{0}^{L} \tilde{C}(\gamma(s)) \sqrt{\beta^{2}(\cos (\theta t) \dot{x}(s)+t \sin (\theta t) \dot{y}(s))^{2}+(\dot{\theta}(s))^{2}} \mathrm{~d} s \\
& =\int_{0}^{L} \tilde{C}(\gamma(s)) f_{t}(\theta) \sqrt{\kappa^{2}+\beta^{2}\left(f_{t}(\theta)\right)^{-3}} \mathrm{~d} s
\end{aligned}
$$

under the side conditions (D.84). So then the gradient of the energy should be linearly dependent on the gradient of the side condition and therefor we set the energy one-form

$$
\psi_{t}=\tilde{C}(x, y, \theta) f_{t}(\theta) \sqrt{\kappa^{2}+\beta^{2}\left(f_{t}(\theta)\right)^{-3}} \mathrm{~d} s+\sum_{i=1}^{3} \lambda_{i}^{t} \theta^{i},
$$

where $\lambda_{1}^{t}, \lambda_{2}^{t}, \lambda_{3}^{t}$ are Lagrange multipliers. Note that $\psi_{t=1}=\sqrt{\kappa^{2}+\beta^{2}} \mathrm{~d} s+\sum_{i=1}^{3} \lambda_{i}^{t} \theta^{i}$ and $\psi_{t=0}=\sqrt{\left(\theta^{\prime}(x)\right)^{2}+\beta^{2}} \mathrm{~d} x+\sum_{i=1}^{3} \lambda_{i}^{t} \theta^{i}$. We consider the affine sub-bundle

$$
Z=\left\{Z_{q} \mid q \in Q\right\} \equiv Q \times T(S E(2))^{*}
$$

of $T^{*}(Q)$ determined by $Z_{q}=\left\{\left.\tilde{C}(x, y, \theta) f_{t}(\theta) \sqrt{\kappa^{2}+\beta^{2}\left(f_{t}(\theta)\right)^{-3}} \mathrm{~d} s\right|_{q} \in I_{q} \subset T_{q}^{*}(Q)\right\}$, which is isomorphic to $Z \equiv Q \times T(S E(2))^{*}$ by isomorphism

$$
\left.(q, \boldsymbol{\lambda}) \leftrightarrow \tilde{C}(x, y, \theta) f_{t}(\theta) \sqrt{\kappa^{2}+\beta^{2}\left(f_{t}(\theta)\right)^{-3}} \sigma \mathrm{d} t\right|_{q}+\left.\sum_{k=1}^{3} \lambda_{k}^{t} \theta^{k}\right|_{q}
$$

Now suppose we have a 1-parameter family of Legendre sub-manifolds $\left\{N_{t}\right\}_{t \in \mathbb{R}}$ within $Z$, this corresponds to a one parameter family of horizontal vector fields on $(S E(2))_{t}$. Then compute the variation of the integrated Lagrangian-form $\psi_{t}$ along $N_{t}$ :

$$
\left.\frac{d}{d t} \int_{N_{t}} \psi_{t}=\int_{N_{t}} \mathcal{L}_{\frac{d}{d t}} \psi_{t}=\int_{N_{t}} \frac{\partial}{\partial t} \psi_{t}=\int_{N_{t}} \frac{\partial}{\partial t}\right\rfloor \mathrm{d} \psi_{t}+\int_{N_{t}} \mathrm{~d}\left(\frac{\partial}{\partial t}\left\lfloor\psi_{t}\right)=\int_{N_{t}} \frac{\partial}{\partial t}\right\rfloor \mathrm{d} \psi_{t}
$$

where we used the well-known Stokes Theorem $\left.\left.\int_{N_{t}} \mathrm{~d}\left(\frac{\partial}{\partial_{t}}\right\rfloor \psi_{t}\right)=\oint_{\partial_{N_{t}}} \frac{\partial}{\partial_{t}}\right\rfloor \psi_{t}=0$ and the formula for Lie derivatives of volume forms along vector fields $\left.\left.\mathcal{L}_{X} A=X\right\rfloor A+d(X\rfloor A\right)$. Consequently, the optimal/characteristic curves are entirely determined by

$$
\left.\gamma^{\prime}(s)\right\rfloor\left.\mathrm{d} \psi_{\mathrm{t}}\right|_{\gamma(s)}=0 \quad \text { for all } s>0 .
$$

But this by definition of the insert operator $\rfloor$ means that $\left.\mathrm{d} \psi_{t}\right|_{\gamma}\left(\gamma^{\prime}, v\right)=0$ for all $v \in T(Z)$, or equivalently formulated

$$
v\rfloor \mathrm{d} \psi_{t}=0 \text { for all } v \in T(Z) .
$$

For further details see [4].

So first we compute the exterior derivative $\mathrm{d} \psi_{t}$ of the Lagrangian form $\psi_{t}$ on $Z$ :

$$
\begin{aligned}
\mathrm{d} \psi_{t} & =\mathrm{d} \lambda_{1}^{t} \wedge \mathrm{d} \theta-\kappa f_{t}(\theta) \sigma \mathrm{d} \lambda_{1}^{t} \wedge \mathrm{d} r-\lambda_{1}^{t} f_{t}(\theta) \sigma \mathrm{d} \kappa \wedge \mathrm{d} r-\lambda_{1}^{t} \kappa f_{t}(\theta) \mathrm{d} \sigma \wedge \mathrm{d} r-\kappa \lambda_{1}^{t} \sigma\left(f_{t}^{\prime}(\theta)\right) \mathrm{d} \theta \wedge \mathrm{d} r \\
& +\sum_{i=1}^{3} \mathcal{A}_{i} \tilde{C} \sqrt{\kappa^{2}+\beta^{2}\left(f_{t}(\theta)\right)^{-3}} f_{t}(\theta) \sigma \mathrm{d} \mathcal{A}^{i} \wedge \mathrm{d} r+\mathrm{d} \lambda_{2}^{t} \wedge \mathrm{d} \mathcal{A}_{2}^{t}+\lambda_{2}^{t} t^{2} \mathrm{~d} \theta \wedge \mathrm{d} \mathcal{A}_{3}^{t}-\frac{\sigma}{\sqrt{f_{t}(\theta)}} \mathrm{d} \lambda_{2}^{t} \wedge \mathrm{d} r \\
& +\frac{f_{t}^{\prime}(\theta)}{2\left(f_{t}(\theta)\right)^{\frac{3}{2}}} \sigma \lambda_{2}^{t} \mathrm{~d} \theta \wedge \mathrm{d} r-\lambda_{2}^{t}\left(f_{t}(\theta)\right)^{-\frac{1}{2}} \mathrm{~d} \sigma \wedge \mathrm{d} r++\mathrm{d} \lambda_{3}^{t} \wedge \mathrm{d} \mathcal{A}_{3}^{t}+\lambda_{3}^{t} \mathrm{~d} \theta \wedge \mathrm{d} \mathcal{A}_{2}^{t} \\
& +\tilde{C} f_{t}(\theta) \sqrt{\kappa^{2}+\beta^{2}\left(f_{t}(\theta)\right)^{-3}} \mathrm{~d} \sigma \wedge \mathrm{d} r+\tilde{C} \frac{d}{d \theta}\left(f_{t}(\theta) \sqrt{\kappa^{2}+\beta^{2}\left(f_{t}(\theta)\right)^{-3}}\right) \sigma \mathrm{d} \theta \wedge \mathrm{d} r \\
& +\tilde{C} \frac{\kappa f_{t}(\theta)}{\sqrt{\kappa^{2}+\beta^{2}\left(f_{t}(\theta)\right)^{-3}}} \sigma \mathrm{d} \kappa \wedge \mathrm{d} r
\end{aligned}
$$

\footnotetext{
${ }^{12}$ If one sets $\tilde{C}=1$ then (D.87) is the same as (C.77). In general we would like to use for respectively $t=1$ the orientation score, and for $t=0$ the Gabor transform of an image to obtain a positive cost-measure $C:(S E(2))_{t} \rightarrow$ $\mathbb{R}^{+}$which we use to define $\tilde{C}=1+C$, so that the optimal curve minimizes both an internal energy on $(S E(2))_{t}$ to guarantee stiffness and an external energy given by the data in the wavelet/Gabor-domain. This will be explained later in more detail in subsection D.2. For now the reader should assume that $\tilde{C}:(S E(2))_{t} \rightarrow[1, \infty)$ is given.
} 
where we used the well-known Maurer-Cartan structural equation, [6]: $\mathrm{dd} \mathcal{A}_{t}^{k}=-\frac{1}{2} \sum_{i, j, k=1}^{3} c_{i j}^{k} \mathrm{~d} \mathcal{A}_{\mathrm{t}}^{\mathrm{i}} \wedge$ $\mathrm{d} \mathcal{A}_{\mathrm{t}}^{\mathrm{j}}$, where $c_{i j}^{k}$ denote the structure constants of the Lie-algebra of $(S E(2))_{t}$, so

$$
\operatorname{dd} \mathcal{A}_{t}^{2}=t^{2} \mathrm{~d} \mathcal{A}_{1}^{t} \wedge \mathrm{d} \mathcal{A}_{t}^{3}, \quad \operatorname{dd} \mathcal{A}_{t}^{3}=-\mathrm{d} \mathcal{A}_{1}^{t} \wedge \mathrm{d} \mathcal{A}_{t}^{2}, \quad \operatorname{dd} \mathcal{A}_{t}^{1}=0 .
$$

and then we explicitly compute the Pfaffian forms (D.88) which should vanish along the characteristic curves (for decent parameterizations satisfying $\gamma^{*} \mathrm{~d} r \neq 0$, with $\sigma \mathrm{d} r=\mathrm{d} s$ ):

$$
\left\{\begin{array}{l}
\partial_{\lambda_{1}^{t}} \mathrm{~d} \psi_{t}=\mathrm{d} \theta-\kappa f_{t}(\theta) \sigma \mathrm{d} r=0 \\
\partial_{\lambda_{2}^{t}} \mathrm{~d} \psi_{t}=\mathrm{d} \mathcal{A}_{t}^{2}-\left(f_{t}(\theta)\right)^{-\frac{1}{2}} \sigma \mathrm{d} r=0 \\
\partial_{\lambda_{3}^{t}} \mathrm{~d} \psi_{t}=\mathrm{d} \mathcal{A}_{t}^{3}=0 \\
\left.\partial_{\sigma}\right\rfloor \mathrm{d} \psi_{t}=\left(\tilde{C} f_{t}(\theta) \sqrt{\kappa^{2}+\beta^{2}\left(f_{t}(\theta)\right)^{-3}}-\lambda_{1}^{t} \kappa f_{t}(\theta)-\lambda_{2}^{t}\left(f_{t}(\theta)\right)^{-\frac{1}{2}}\right) \mathrm{d} r=0 \\
\partial_{\kappa} \mathrm{d} \psi_{t}=\left(\tilde{C} \kappa\left(\kappa^{2}+\beta^{2}\left(f_{t}(\theta)\right)^{-3}\right)^{-1 / 2}-\lambda_{1}^{t} f_{t}(\theta)\right) \sigma \mathrm{d} r=0 \\
\left.-\partial_{\theta}\right\rfloor \mathrm{d} \psi_{t}=\mathrm{d} \lambda_{1}^{t}+\kappa \lambda_{1}^{t} \sigma\left(f_{t}^{\prime}(\theta)\right) \mathrm{dr}-t^{2} \mathrm{~d} \mathcal{A}_{t}^{3}-\frac{1}{2} \frac{f_{t}^{\prime}(\theta)}{f_{t}(\theta)^{\frac{3}{2}}} \sigma \lambda_{2}^{t} \mathrm{~d} r \\
-\left(\mathcal{A}_{1} \tilde{C}\right) f_{t}(\theta) \sqrt{\kappa^{2}+\beta^{2}\left(f_{t}(\theta)\right)^{-3}} \mathrm{~d}+\lambda_{3}^{t} \mathrm{~d} \mathcal{A}_{t}^{2}+\tilde{C} \frac{d}{d \theta}\left(f_{t}(\theta) \sqrt{\kappa^{2}+\beta^{2}\left(f_{t}(\theta)\right)^{-3}}\right) \sigma \mathrm{d} r=0 \\
-\mathcal{A}_{2}^{t} \mathrm{~d} \psi_{t}=\mathrm{d} \lambda_{2}^{t}-\lambda_{3}^{t} \mathrm{~d} \theta-\left(\mathcal{A}_{2} \tilde{C}\right) f_{t}(\theta) \sqrt{\kappa^{2}+\beta^{2}\left(f_{t}(\theta)\right)^{-3}} \mathrm{~d} s=0 \\
-\mathcal{A}_{3}^{t} \mathrm{~d} \psi_{t}=\mathrm{d} \lambda_{3}^{t}+\lambda_{2}^{t} t^{2} \mathrm{~d} \theta-\left(\mathcal{A}_{3} \tilde{C}\right) f_{t}(\theta) \sqrt{\kappa^{2}+\beta^{2}\left(f_{t}(\theta)\right)^{-3}} \mathrm{~d} s=0
\end{array}\right.
$$

where we use short notation $\tilde{C}=\tilde{C}(\gamma(r))=\tilde{C}(x(r), y(r), \theta(r)), \mathcal{A}_{i} \tilde{C}=\left(\mathcal{A}_{i}^{t} \tilde{C}\right)(\gamma(r)), i=1,2,3$.

The sixth Pfaffian form can be considerably simplified is one uses the other Pfaffian forms. After a brief computation one finds:

$$
\left.-\partial_{\theta}\right\rfloor \mathrm{d} \psi_{t}=\mathrm{d} \lambda_{1}^{t}-\left(\mathcal{A}_{1} \tilde{C}\right) f_{t}(\theta) \sqrt{\kappa^{2}+\beta^{2}\left(f_{t}(\theta)\right)^{-3}} \mathrm{~d} s+\lambda_{3}^{t} \mathrm{~d} \mathcal{A}_{t}^{2}=0
$$

We introduce the variable $\xi_{t}$

$$
\xi_{t}=x \cos (\theta t)+y t \sin (\theta t) \text { for } t \in(0,1]
$$

which runs along $\mathcal{A}_{2}^{t}$ in the spatial plane and solve for $\lambda_{1}^{t}, \lambda_{2}^{t}, \lambda_{3}^{t}$ in the Pfaffian system (D.89):

$$
\lambda_{1}^{t}=\tilde{\mathcal{C}}\left(\frac{\frac{d \theta}{d \xi}}{\sqrt{\left(\frac{d \theta}{d \xi_{t}}\right)^{2}+\beta^{2}}}\right), \lambda_{2}^{t}=\tilde{\mathcal{C}}\left(\frac{\beta^{2}}{\sqrt{\left(\frac{d \theta}{d \xi_{t}}\right)^{2}+\beta^{2}}}\right), \lambda_{3}^{t}=-\frac{\mathrm{d} \lambda_{1}^{t}}{\mathrm{~d} \xi_{t}}+\left(\mathcal{A}_{1} \tilde{C}\right) \sqrt{\left(\frac{d \theta}{d \xi_{t}}\right)^{2}+\beta^{2}}
$$

Here we note that in the case $\tilde{C}=1$ the variable $\lambda_{3}^{t}$ variable represents the Euclidean curvature of the graph $\left\{\left(\xi_{t}, \beta^{-1} \theta\left(\xi_{t}\right)\right) \mid \xi_{t} \in\left[0, \xi_{\max }\right)\right\}$. In case $\tilde{C}=1$ and $t \downarrow 0$ we have $\xi_{t}=x$ and indeed we found $\lambda_{3}^{t=0}=\beta c$ for some constant $c$, recall (C.81).

By employing Noether's theorem and an invariance group of symmetries of $\psi$ (which is defined as a group acting on $Q$ such that the induced action $\eta$ on $T^{*}(Q)$ satisfies $\eta_{g}(Z)=Z$ for all $g \in G$ ) as will briefly explain next. In our case the action $\eta$ on $T^{*}(Q)$ is induced by the left action of $(S E(2))_{t}$ on itself) of the minimization problem.

Noether's theorem says that the momentum mapping $m: Z \rightarrow S E(2)_{t}^{*}$ given by

$$
\langle m(p), \xi\rangle=(\xi\rfloor \psi)(p), \quad p \in Z,
$$

is constant along the characteristic curves. Note that computation of the Lie-derivative of $\psi$ along a characteristic curve gives

$$
\left.\left.\left.0=\mathcal{L}_{\xi} \mathcal{A}=\mathcal{A}\right\rfloor \mathrm{d} \psi+d(\mathcal{A}\rfloor \psi\right)=\mathcal{A}\right\rfloor \mathrm{d} \psi, \quad \text { for all left-invariant vector fields } \mathcal{A} \in \mathcal{L}\left((S E(2))_{t}\right),
$$

which coincides with the last three equalities in (D.88).

The co-adjoint representation of $(S E(2))_{t}$ acting on the dual of its Lie-algebra $\left(T\left((S E(2))_{t}\right)\right)^{*}$ is given by

$$
\left\langle\left(\operatorname{Ad}_{g^{-1}}\right)^{*} \lambda, \xi\right\rangle=\left\langle\lambda, \operatorname{Ad}_{g} \xi\right\rangle
$$


where Ad is the well-known adjoint representation of the group $(S E(2))_{t}$ acting on its own Lie-algebra.

More explicitly the co-adjoint representation of $(S E(2))_{t}$ is given by

$$
\begin{aligned}
\left(\operatorname{Ad}_{g^{-1}}\right)^{*}\left(\lambda_{1}^{t} \mathrm{~d} \mathcal{A}_{t}^{1}+\lambda_{2}^{t} \mathrm{~d} \mathcal{A}_{t}^{2}+\lambda_{3}^{t} \mathrm{~d} \mathcal{A}_{t}^{3}\right) & =\left(\lambda_{1}^{t}+\lambda_{2}^{t} t y-x \lambda_{3}^{t}\right) \mathrm{d} \mathcal{A}_{t}^{1}+\left(\lambda_{2}^{t} \cos (\theta t)+\lambda_{3}^{t} \frac{\sin (\theta t)}{t}\right) \mathrm{d} \mathcal{A}_{t}^{2} \\
& +\left(\cos (\theta t) \lambda_{3}^{t}-t \lambda_{2}^{t} \sin (\theta t)\right) \mathrm{d} \mathcal{A}_{t}^{3}
\end{aligned}
$$

As a result the co-adjoint orbits of $(S E(2))_{t}$ coincide with the cilinders given by $t^{2}\left(\lambda_{2}^{t}\right)^{2}+\left(\lambda_{3}^{t}\right)^{2}=$ constant. These co-adjoint orbits are highly relevant for the computation of the geodesics in $(S E(2))_{t}$ as the momentum mapping is invariant under the co-adjoint representation

$$
m\left(\eta_{g}(p)\right)=\left(\operatorname{Ad}_{g^{-1}}\right)^{*} m(p),
$$

where $\eta_{g}\left(p=\left(g^{\prime}, \kappa, \sigma, \lambda\right)\right)=\left(g g^{\prime}, \kappa, \sigma,\left(\operatorname{Ad}_{g^{-1}}\right)^{*} \lambda\right)$, so that $\eta_{g}^{*} \psi=\psi$ and $\left(\eta_{g}\right) \xi=\left(\operatorname{Ad}_{g}\right) \xi$, form which it indeed follows that

$$
\begin{aligned}
\left\langle m\left(\eta_{g} p\right), \xi\right\rangle & \left.=(\xi\rfloor \psi)\left(\eta_{g}(p)\right)=\left(\left(\eta_{g}\right)_{*} \xi\right\rfloor\left(\eta_{g}\right)^{*} \psi\right)(p) \\
& \left.\left.=\left(\left(\operatorname{Ad}_{g}\right) \xi\right\rfloor\left(\eta_{g}\right)^{*} \psi\right)(p)=(\xi\rfloor\left(\operatorname{Ad}_{g^{-1}}\right)_{*} \psi\right)(p) \\
& =\left\langle\left(\operatorname{Ad}_{g^{-1}}\right)_{*} m(p), \xi\right\rangle
\end{aligned}
$$

for all $\xi \in T\left((S E(2))_{t}\right)$. Consequently, the geodesics (or characteristic curves) are contained in the coadjoint orbits and we get the following preservation law that holds along the characteristic curves:

$$
t^{2}\left(\lambda_{2}^{t}\right)^{2}+\left(\lambda_{3}^{t}\right)^{2}=c^{2} \beta^{2} \geq 0, \quad c>0 .
$$

Moreover, we have another preservation law

$$
\beta^{2} \tilde{C}^{2}=\beta^{2}\left(\lambda_{1}^{t}\right)^{2}+\left(\lambda_{2}^{t}\right)^{2},
$$

which directly follows by (D.91).

Now that we have used the first six Pfaffian forms within (D.89) to obtain the formulae (D.91), we will use these expressions to derive the actual solution curves $\gamma=(x, y, \theta)$. Therefor we define the following diagonal matrix $S_{t}$ :

$$
S_{t}=\left(\begin{array}{ccc}
t & 0 & 0 \\
0 & t^{-1} & 0 \\
0 & 0 & t
\end{array}\right)
$$

and we note that the last 3 equations of (D.89) may be re-written in vector form as

$$
\mathrm{d} \hat{\lambda}^{t}=\hat{\lambda}^{t} S_{t}^{-1}\left(\gamma^{-1} \mathrm{~d} \gamma\right) S_{t}+\widehat{\nabla \tilde{C}} \mathrm{~d} p
$$

where we use short-notation for the row-vector $\hat{\lambda}^{t}=\left(-\lambda_{3}^{t}, \lambda_{2}^{t}, \lambda_{1}^{t}\right)$ and the left-invariant gradient $\widehat{\nabla \tilde{C}}=$ $\left(-\mathcal{A}_{3}^{t} \tilde{C}, \mathcal{A}_{2}^{t} \tilde{C}, \mathcal{A}_{1}^{t} \tilde{C}\right)$ and where $\gamma^{-1} \mathrm{~d} \gamma$ denotes the Cartan-connection on $(S E(2))_{t}$ :

$$
\gamma^{-1} \mathrm{~d} \gamma=\left(\begin{array}{ccc}
\cos (\theta t) & -t \sin (\theta t) & x \\
\frac{\sin \theta t}{t} & \cos \theta t & y \\
0 & 0 & 1
\end{array}\right) \quad \mathrm{d}\left(\begin{array}{ccc}
\cos (\theta t) & -t \sin (\theta t) & x \\
\frac{\sin \theta t}{t} & \cos \theta t & y \\
0 & 0 & 1
\end{array}\right)=\left(\begin{array}{ccc}
0 & -t^{2} \mathrm{~d} \theta & \mathrm{d} \mathcal{A}_{t}^{2} \\
\mathrm{~d} \theta & 0 & \mathrm{~d} \mathcal{A}_{t}^{3} \\
0 & 0 & 0
\end{array}\right)
$$

and where $p$ denotes the arc-length parameter in $(S E(2))_{t}$, such that

$$
\begin{aligned}
& \mathrm{d} p=f_{t}(\theta) \sqrt{\kappa^{2}+\beta^{2}\left(f_{t}(\theta)\right)^{-3}} \mathrm{~d} s=\sqrt{\left(\frac{d \theta}{d \xi_{t}}\right)^{2}+\beta^{2}} \mathrm{~d} \xi_{t} \\
& \lambda_{1}^{t}(p)=\tilde{C}(\gamma(p)) \frac{d \theta}{d p} \\
& \lambda_{2}^{t}(p)=\beta^{2} \tilde{C}(\gamma(p)) \frac{d \xi^{t}}{d p}
\end{aligned}
$$

Now straightforward manipulation on (D.95) yields

$$
\begin{aligned}
& \frac{d \hat{\lambda}^{t}}{d p}=\hat{\lambda}^{t}\left(S_{t}\right)^{-1}\left(\gamma^{-1} \frac{\mathrm{d} \gamma}{\mathrm{d} p}\right) S_{t}+\widehat{\nabla \tilde{C}}(\gamma) \Leftrightarrow \\
& \frac{d \hat{\lambda}^{t}}{d p}\left(\gamma S_{t}\right)^{-1}-\hat{\lambda}^{t}\left(\gamma S_{t}\right)^{-1} \frac{\mathrm{d}\left(\gamma S_{t}\right)}{\mathrm{d} p}\left(\gamma S_{t}\right)^{-1}=\widehat{\nabla \tilde{C}}(\gamma) \cdot\left(\gamma S_{t}\right)^{-1} \Leftrightarrow \\
& \frac{d}{d p}\left(\hat{\lambda}^{t}(p)\left(\gamma(p) S_{t}\right)^{-1}\right)=\widehat{\nabla \tilde{C}}(\gamma(p)) \cdot\left(\gamma(p) S_{t}\right)^{-1}, \text { for all } p \in\left(0, p_{\gamma}^{\max }\right) \Leftrightarrow \\
& \hat{\lambda}^{t}(p)=\hat{\mu} \cdot\left(\gamma(p) S_{t}\right)+\int_{0}^{p} \widehat{\nabla \tilde{C}}(\gamma(\tilde{p})) \cdot S_{t}^{-1}(\gamma(\tilde{p}))^{-1} \gamma(p) S_{t} \mathrm{~d} \tilde{p}, \text { for all } p \in\left(0, p_{\gamma}^{\max }\right),
\end{aligned}
$$


where the constants $\hat{\mu}^{t}=\left(-\mu_{3}^{t}, \mu_{1}^{t}, \mu_{2}^{t}\right)$ are determined by $\hat{\lambda}^{t}(0)=\hat{\mu} \cdot\left(\gamma(0) S_{t}\right)$.

Now we use left-invariance of the optimization problem (D.87) and map the horizontal curve $\gamma$ onto the horizontal curve $\tilde{\gamma}$

$$
\gamma=(x, y, \theta)=h_{0}^{-1} \tilde{\gamma}=h_{0}^{-1} \tilde{\gamma},
$$

where $h_{0}^{-1} \in(S E(2))_{t}$ is given by

$$
h_{0}^{-1}=\left(\begin{array}{ccc}
\frac{-t \mu_{3}^{t}}{\beta c} & \frac{-t \mu_{2}^{t}}{\beta c} & \frac{t^{2} \mu_{1}^{t} \mu_{3}^{t}}{c^{2} \beta^{2}} \\
\frac{t^{-1} \mu_{2}^{t}}{\beta c} & \frac{-t \mu_{3}^{t}}{\beta c} & \frac{-\mu_{1}^{t} \mu_{2}^{t}}{c^{2} \beta^{2}} \\
0 & 0 & 1
\end{array}\right) \text { so that } \hat{\mu} \cdot h_{0}^{-1}=\left(\frac{\beta c}{t}, 0,0\right) \text {. }
$$

Here we note that

$$
\left(\lambda_{3}^{t}\right)^{2}+t^{2}\left(\lambda_{2}^{t}\right)^{2}=\beta^{2} c^{2} \Leftrightarrow\left(\mu_{2}^{t}\right)^{2}+\left(\mu_{3}^{t}\right)^{2} t^{2}=\beta^{2} c^{2},
$$

from which we indeed deduce that $h_{0}^{-1} \in(S E(2))_{t}$.

Now substitution of (D.98) into (D.97) using (D.99) yields

$$
\begin{aligned}
& \left(-\lambda_{3}^{t}(p), \lambda_{2}^{t}(p), \lambda_{1}^{t}(p)\right)=\left(\begin{array}{ccc}
\frac{\beta c}{t} & 0 & 0
\end{array}\right) \tilde{\gamma}(p) S_{t} \\
& +\int_{0}^{p}\left(-\mathcal{A}_{3} \tilde{C}\left(h_{0}^{-1} \tilde{\gamma}(p)\right) \quad \mathcal{A}_{2} \tilde{C}\left(h_{0}^{-1} \gamma(p)\right) \quad \mathcal{A}_{1} \tilde{C}\left(h_{0}^{-1} \tilde{\gamma}(p)\right)\right) S_{t}^{-1}(\tilde{\gamma}(\tilde{p}))^{-1} \tilde{\gamma}(p) S_{t} \mathrm{~d} \tilde{p}
\end{aligned}
$$

In the general case where the cost-function $\tilde{C}:(S E(2))_{t} \rightarrow \mathbb{R}^{+}$is non-constant, these implicit equations are only implicit in $\tilde{\gamma}$ and they are relatively intangible. For example we have

$$
\begin{aligned}
& \tilde{x}(p)=\frac{1}{\beta c}\left(\tilde{C}(\gamma(p)) \frac{d \theta}{d p}-\int_{0}^{p} \tilde{C}_{\theta}(\gamma(\tilde{p})) S_{t}^{-1}(\gamma(\tilde{p}))^{-1} \gamma(p) S_{t} \mathrm{~d} \tilde{\mathrm{p}}\right) \\
& \tilde{x}^{\prime}\left(\xi_{t}\right)=-\frac{1}{\beta c} \lambda_{3}\left(\xi_{t}\right)-\int_{0}^{\xi_{t}} \tilde{\mathcal{C}}_{\theta}\left(\gamma\left(\tilde{\xi}_{t}\right)\right) S_{t}^{-1}\left(\gamma\left(\tilde{\xi}_{t}\right)\right)^{-1} \sqrt{\left(\tilde{\theta}^{\prime}\left(\tilde{\xi}_{t}\right)\right)^{2}+\beta^{2}} \mathrm{~d} \tilde{\xi}_{t} \cdot \gamma^{\prime}\left(\xi_{t}\right) S_{t}
\end{aligned}
$$

from which we conclude that the use of the $(\tilde{x}, \tilde{y}, \tilde{\theta})$-coordinates only pays off for the case where $\tilde{C}$ is constant.

\section{D.1 The case of a constant cost-function $\tilde{\mathcal{C}}$}

If we set $\tilde{\mathcal{C}}=1$, then

$$
\tilde{\mathcal{C}}=1 \Rightarrow \lambda_{3}\left(\xi_{t}\right)=-\dot{\lambda}_{1}\left(\xi_{t}\right)=-\frac{d \lambda_{1}}{d \xi_{t}}\left(\xi_{t}\right)
$$

and consequently the conservation-law (D.94) reduces to

$$
t^{2} \beta^{2}\left(1-\left(\lambda_{1}^{t}\right)^{2}\right)+\left(\dot{\lambda}_{1}^{t}\right)^{2}=\beta^{2} c^{2},
$$

so that by differentiation we find

$$
\ddot{\lambda}_{1}^{t}=t^{2} \beta^{2} \lambda_{1}^{t} \text {. }
$$

For a typical phase-plot in the $\lambda_{1}^{t}$ and $\lambda_{3}^{t}$ for the case $t=1$ see figure 12 . The phase plots for the cases $0<t<1$ look similar, where the straight orbits coincide with $c=t$. The phase plot in the limiting case

$t \downarrow 0$ looks different, it is a straight horizontal line, since $\lambda_{3}^{t=0}=-\frac{d \lambda_{1}}{d x}=\beta c$.

The linear second order ODE (D.101) has the following solutions

$$
\lambda_{1}^{t}\left(\xi_{t}\right)=z_{0} \cosh \left(\beta t \xi_{t}\right)+\frac{z_{0}^{\prime}}{\beta t} \sinh \left(\beta t \xi_{t}\right), \quad z_{0}, z_{0}^{\prime} \in \mathbb{R} .
$$

From which we can compute the other local multipliers as well

$$
\begin{aligned}
& \lambda_{2}^{t}\left(\xi_{t}\right)=\beta \sqrt{1-\left(\lambda_{1}^{t}\left(\xi_{t}\right)\right)^{2}}, \\
& -\lambda_{3}^{t}\left(\xi_{t}\right)=\dot{\lambda}_{1}^{t}\left(\xi_{t}\right)=z_{0} \beta t \sinh \left(\beta t \xi_{t}\right)+z_{0}^{\prime} \cosh \left(\beta t \xi_{t}\right) .
\end{aligned}
$$



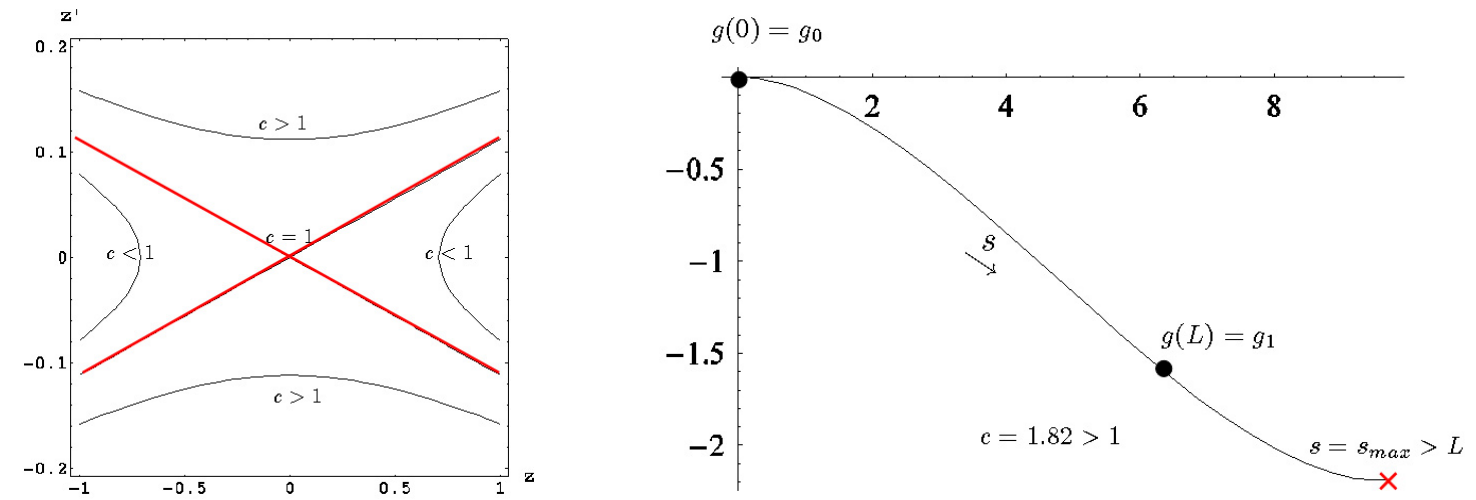

Figure 12: The case $t=1,(S E(2))_{t=1}=S E(2)$, where $z=\lambda_{1}$. Left: A phase plot of $z(s)=\frac{\kappa(s)}{\kappa^{2}(s)+\epsilon}$ and $\dot{z}(s)$ clearly indeed reveals that all paths will eventually end up at $z=1$ where solutions brake down because of infinite curvature. Except for the cases where the initial condition is such that $c=\sqrt{1-\epsilon^{-1}(\dot{z}(0))^{2}-(z(0))^{2}}=1$ then solutions stay at $c=1$ but reach the line $z=1$ only for $s \rightarrow \infty$. Right: These infinite curvature singularities always take place at $s_{\max }>L$, so this causes no problems in our exact analytic solutions (in contrast to the numerical shooting algorithm [17].).

Consequently, we find

$$
\begin{aligned}
& \tilde{x}\left(\xi_{t}\right)=\frac{z_{0}}{\beta c} \cosh \left(\beta t \xi_{t}\right)+\frac{z_{0}^{\prime}}{\beta^{2} t c} \sinh \left(\beta t \xi_{t}\right), \\
& \tilde{y}\left(\xi_{t}\right)=\tilde{y}_{0}+\frac{1}{c} \int_{0}^{\xi_{t}} \sqrt{1-\beta^{2} c^{2} \tilde{x}^{2}(\tau)} \\
& \tilde{\theta}\left(\xi_{t}\right)=t^{-1} \arccos \left\{\frac{z_{0} t}{c} \sinh \left(\beta t \xi_{t}\right)+\frac{z_{0}^{\prime}}{\beta c} \cosh \left(\beta t \xi_{t}\right)\right\} .
\end{aligned}
$$

Now by means of left-invariance we suppose without loss of generality that $\gamma(0)=(0,0,0), \gamma\left(\xi_{\max }\right)=$ $\left(x_{1}, y_{1}, \theta_{1}\right)$, in which case we can easily express the parameters $\mu_{1}^{t}, \mu_{2}^{t}, \mu_{3}^{t}, \xi_{\text {max }}^{t}, \tilde{y}_{0}, c$ in $z_{0}$ and $z_{0}^{\prime}$ :

$$
\begin{array}{ll}
\mu_{3}^{t}=-z_{0}^{\prime} t^{-1}, & c=\sqrt{t^{2}\left(1-\left(z_{0}\right)^{2}\right)+\frac{\left(z_{0}^{\prime}\right)^{2}}{\beta^{2}}} \\
\mu_{2}^{t}= \pm t \beta \sqrt{1-\left(z_{0}\right)^{2}}, & \tilde{y}_{0}=0 \\
\mu_{1}^{t}=z_{0}, & \xi_{t}^{\max }=\frac{1}{\beta t} \log \left(\frac{x_{1} \pm \sqrt{\left(x_{1}\right)^{2}-\left(\frac{z_{0}^{2}}{\beta^{2} c^{2}}-\frac{\left(z_{0}^{\prime}\right)^{2}}{\beta^{4} t^{2} c^{2}}\right)}}{\frac{z_{0}}{\beta c}+\frac{z_{0}^{\prime}}{\beta^{2} t c}}\right) .
\end{array}
$$

Here we note that only for $t=1$ we have $\xi_{t=1}=s$ equals the arc-length parameter $s>0$ in which case we have $\xi_{t=1}^{\max }=L$, where $L$ is the total length of the projected curve $x=P_{\mathbb{R}^{2}} \gamma$ in the spatial plane.

Finally, we determine the remaining unknowns $z_{0}$ and $z_{0}^{\prime}$ (using (D.98)) by

$$
\frac{t^{2} \mu_{1}^{t} \mu_{3}^{t}}{c^{2} \beta^{2}}-\frac{t \mu_{3}^{t}}{c \beta} \tilde{x}\left(\xi_{\text {max }}^{t}\right)-\frac{t \mu_{2}^{t}}{c \beta} \tilde{y}\left(\xi_{\text {max }}^{t}\right)=x_{1}, \quad \frac{-\mu_{1}^{t} \mu_{2}^{t}}{c^{2} \beta^{2}}+\frac{t^{-1} \mu_{2}^{t}}{c \beta} \tilde{x}\left(\xi_{\text {max }}^{t}\right)-\frac{t \mu_{3}^{t}}{c \beta} \tilde{y}\left(\xi_{\text {max }}^{t}\right)=y_{1} .
$$

For a specific plot of a geodesic in $(S E(2))_{t=1}=S E(2)$ (the domain of an orientation score) see Figure 13

However, for optimal curves in the Heisenberg group $H_{3}$ (the domain of Gabor transforms) we must consider the case $\tilde{C}=1$ and $t \downarrow 0$. Recall, see (C.81), that in this particular case $\lambda_{3}^{0}=\lambda_{3}^{t \downarrow 0}=-\beta c$ is constant, yielding solutions (C.82), where we recall (C.75). Furthermore, if $t \downarrow 0$ one has

$$
t \downarrow 0 \Rightarrow\left\{\begin{array}{l}
\theta \rightarrow \tilde{\theta}, \\
x \rightarrow \tilde{x}+\frac{1}{\beta c} \tan \phi|\cos \phi|, \\
y \rightarrow \frac{1}{c}|\cos \phi|+\tilde{y}-\frac{\tan \phi \cos ^{2} \phi}{\beta c^{2}} .
\end{array}\right.
$$

Consequently, since $(S E(2))_{t \downarrow 0}=(S E(2))_{0}=H_{3}$ we must have

$$
\mu_{2}^{t} \sim t \beta|\cos \phi|, \quad \mu_{3}^{t} \sim-\frac{c \beta}{t}, \quad \mu_{1}^{t} \sim-\tan \phi|\cos \phi| t^{-1}, \quad 0<t<<1,
$$



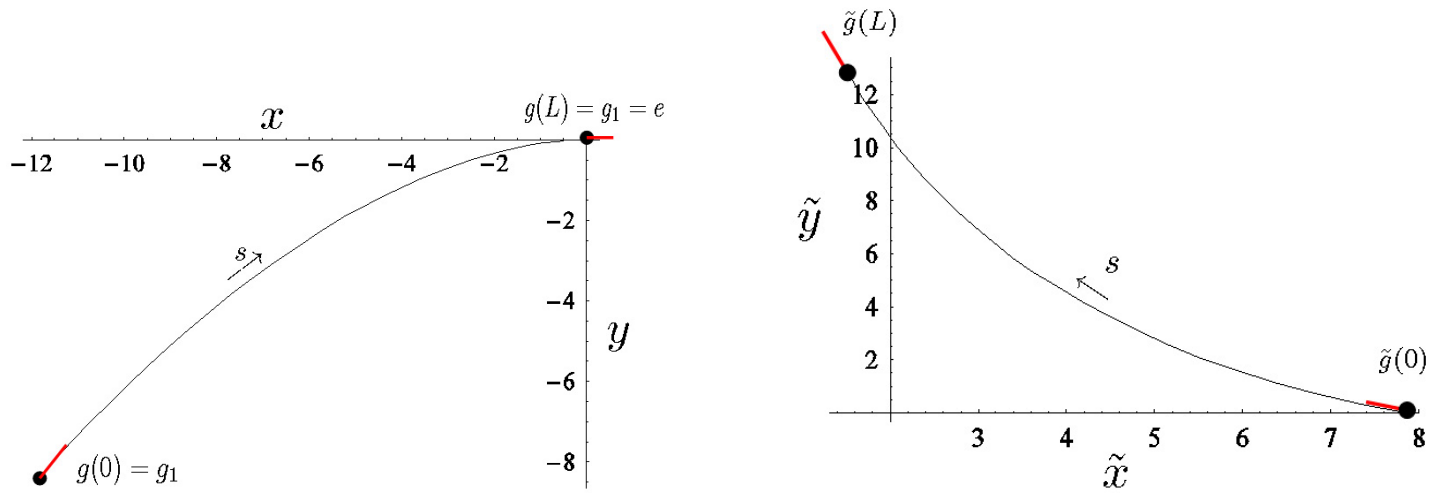

Figure 13: Left figure: Illustration of a geodesic $s \mapsto g(s)$ computed by (D.102) and (D.98) and its affine relative $s \mapsto \tilde{g}(s)=h_{0}^{-1} g(s)$. Parameter settings $x_{0}=-11.868, y_{0}=-8.44337, \theta_{0}=51.95^{\circ}$, $x_{1}=y_{1}=\theta_{1}=0, L=15, \epsilon=0.0125, z_{0}=-0.1641, z_{0}^{\prime}=0.0183, c=1$.

so that indeed (recall (C.82)):

$$
\begin{array}{lll}
\lambda_{1}^{0}=\frac{\theta^{\prime}(x)}{\sqrt{\left(\theta^{\prime}(x)\right)^{2}+\beta^{2}}} & =\lim _{t \downarrow 0} \lambda_{1}^{t}=\lim _{t \downarrow 0}\left\{-\mu_{3}^{t} x t+\mu_{2}^{t} y t+\mu_{1}^{t} t\right\} & =c \beta x-\sin \phi \frac{|\cos \phi|}{\cos \phi}, \\
\lambda_{2}^{0}=\frac{\beta^{2}}{\sqrt{\left(\theta^{\prime}(x)\right)^{2}+\beta^{2}}} & =\lim _{t \downarrow 0} \lambda_{2}^{t}=\lim _{t \downarrow 0}\left\{\mu_{3}^{t} \sin (\theta t)+\frac{\mu_{2}^{t} \cos (\theta t)}{t}\right\} & =-c \beta \theta+\beta|\cos \phi|, \\
\lambda_{3}^{0}=-\frac{d}{d x}\left\{\frac{\theta^{\prime}(x)}{\sqrt{\left(\theta^{\prime}(x)\right)^{2}+\beta^{2}}}\right\} & =\lim _{t \downarrow 0} \lambda_{3}^{t}=\lim _{t \downarrow 0}\left\{-\mu_{3}^{t} t \cos (\theta t)+\mu_{2}^{t} \sin (\theta t)\right\} & =-c \beta .
\end{array}
$$

\section{D.2 The case of a non-constant cost-function $\tilde{C}$ and optimal curves in Gabor transforms $(t=0)$ and Orientation scores $(t=1)$}

Suppose we are given the enhanced orientation score or the enhanced/diffused Gabor transform $g \mapsto$ $\Phi_{s}\left(\mathcal{W}_{\psi}(f)\right)(g)$, where $\Phi_{s}$ denotes a non-linear left-invariant diffusion operator with stopping time $s>0$ as we described for Gabor-transforms in Section 7 and as we described for orientation scores [12](part II), [17]. Then we set

$$
\tilde{\mathcal{C}}(x, y, \theta)=1+\varepsilon \mathcal{C}(x, y, \theta) \geq 1 \text {, with } \mathcal{C}(x, y, \theta)=e^{-\beta\left|\Phi_{s}\left(\mathcal{W}_{\psi}(f)\right)(x, y, \theta)\right|},
$$

for some parameter $\varepsilon>0$ which is a classical, balance-parameter between the internal energy (in order to require "stiffness" of the curve) and external energy (in order to fit the data) of the curve and some decay parameter $\beta>0$, which naturally occur in so-called "snake-algorithms" in images, like for example [32]. However, in contrast to the common approaches on snakes, such as [32], we consider "snakes" in the enhanced/diffused wavelet-Gabor transforms of images rather than snakes in the images themselves.

More precisely the decomposition of the energy (C.78) in internal energy and external energy is given by

$$
\begin{aligned}
\mathcal{E}(\gamma) & =\int_{0}^{L} \tilde{\mathcal{C}}(\gamma(s)) f_{t}(\theta) \sqrt{\kappa^{2}(s)+\beta^{2}\left(f_{t}(\theta)\right)^{-3}} \mathrm{~d} s=\int_{0}^{\xi_{t}^{\max }}\left(1+\varepsilon \mathcal{C}\left(\gamma\left(\xi_{t}\right)\right)\right) \sqrt{\left(\frac{d \theta}{d \xi_{t}}\right)^{2}+\beta^{2}} \mathrm{~d} \xi_{t} \\
& =\int_{0}^{p_{\gamma}^{\max }} 1 \mathrm{~d} p+\varepsilon \int_{0}^{p_{\gamma}^{\max }} \mathcal{C}(\gamma(p)) \mathrm{d} p=: \mathcal{E}^{\operatorname{int}}(\gamma)+\varepsilon \mathcal{E}^{\operatorname{ext}}(\gamma), \quad \varepsilon>0,
\end{aligned}
$$

where we recall that $p>0$ resp. $s>0$ denotes arc-length in $(S E(2))_{t}$ resp. $\mathbb{R}^{2}$ and where $p_{\gamma}^{\max }$ denotes the total $S E(2))_{t}$ arc-length of curve $\gamma$.

Along (horizontal) characteristic curves $\gamma=(x, y, \theta)$, which minimize (D.104), we have the following ODE-system

$$
\left\{\begin{array}{l}
x^{\prime}\left(\xi_{t}\right)=\cos \left(t \theta\left(\xi_{t}\right)\right) \\
y^{\prime}\left(\xi_{t}\right)=t^{-1} \sin \left(t \tilde{\theta}\left(\xi_{t}\right)\right) \\
\theta^{\prime}\left(\xi_{t}\right)=\frac{\left(\lambda_{2}^{t}\right)^{\prime}\left(\xi_{t}\right)-\left(\mathcal{A}_{2} C\right)\left(\gamma\left(\xi_{t}\right)\right) \sqrt{\left(\theta^{\prime}\left(\xi_{t}\right)\right)^{2}+\beta^{2}}}{\lambda_{3}^{t}\left(\xi_{t}\right)}
\end{array}\right.
$$


Where we note that the first equations follow by horizontality, whereas the last equation corresponds to the one but last equation in (D.89).

Now by multiplying the last equality in (D.89) by $\lambda_{3}$ and multiplying the one but last equation by $t^{2} \lambda_{2}$ and adding the results we find that along the characteristic curve $\gamma$ one has

$$
\left(t^{2} \lambda_{2}^{t}\left(\xi_{t}\right) \mathcal{A}_{2} \tilde{C}\left(\gamma\left(\xi_{t}\right)\right)+\lambda_{3}^{t}\left(\xi_{t}\right) \mathcal{A}_{3} \tilde{C}\left(\gamma\left(\xi_{t}\right)\right)\right) \mathrm{d} p=0=t^{2} \lambda_{2}^{t} \mathrm{~d} \lambda_{t}^{2}+\lambda_{3}^{t} \mathrm{~d} \lambda_{t}^{3}=0
$$

so for decent parameterizations we find for all $\xi_{t} \in\left(0, \xi_{\max }^{t}\right)$ that

$$
t^{2} \lambda_{2}^{t}\left(\xi_{t}\right) \mathcal{A}_{2} \tilde{C}\left(\gamma\left(\xi_{t}\right)\right)+\lambda_{3}^{t}\left(\xi_{t}\right) \mathcal{A}_{3} \tilde{C}\left(\gamma\left(\xi_{t}\right)\right)=0 .
$$

So in particular if $t \downarrow 0$ we see that $\mathcal{A}_{3}^{0} \tilde{C}(\gamma)=\partial_{y} \tilde{C}(\gamma)=0$.

Now it directly follows from (D.106) and (D.93) that

$$
\lambda_{2}\left(\xi_{t}\right)=-\left.c \beta \frac{\mathcal{A}_{3} \tilde{C}}{\sqrt{t^{2}\left(\mathcal{A}_{3} \tilde{C}\right)^{2}+t^{4}\left(\mathcal{A}_{2} \tilde{C}\right)^{2}}}\right|_{\xi_{t}}, \quad \lambda_{3}\left(\xi_{t}\right)=\left.c \beta \frac{\mathcal{A}_{2} \tilde{C}}{\sqrt{t^{2}\left(\mathcal{A}_{3} \tilde{C}\right)^{2}+t^{4}\left(\mathcal{A}_{2} \tilde{C}\right)^{2}}}\right|_{\xi_{t}},
$$

for $t>0$, as long as $\left(\mathcal{A}_{2} \mathcal{C}\left(\gamma\left(\xi_{t}\right)\right), \mathcal{A}_{3} \mathcal{C}\left(\gamma\left(\xi_{t}\right)\right)\right) \neq(0,0)$.

Now we substitute the formula for $\lambda_{2}$ recall (D.91) into (D.105) and brief calculation yields (where all derivatives are taking with respect to $\xi_{t}$ )

$$
\begin{aligned}
\theta^{\prime} & =\frac{1}{\lambda_{3}^{t}}\left(\tilde{C}\left(\frac{\beta^{2}}{\left(\theta^{\prime}\right)^{2}+\beta^{2}}\right)^{\prime}+\tilde{C}^{\prime}\left(\frac{\beta^{2}}{\left(\theta^{\prime}\right)^{2}+\beta^{2}}\right)-\left(\left(\theta^{\prime}\right)^{2}+\beta^{2}\right) \mathcal{A}_{2} \tilde{\mathcal{C}}\right) \\
& =\frac{1}{\lambda_{3}^{t}}\left(\tilde{\mathcal{C}} \frac{-\beta^{2} \theta^{\prime} \theta^{\prime \prime}}{\left(\left(\theta^{\prime}\right)^{2}+\beta^{2}\right)^{\frac{3}{2}}}+\frac{\theta^{\prime} \tilde{\mathcal{C}}_{\theta}-\left(\theta^{\prime}\right)^{2} \mathcal{A}_{2} \tilde{\mathcal{C}}}{\left(\left(\theta^{\prime}\right)^{2}+\beta^{2}\right)^{\frac{1}{2}}}\right)
\end{aligned}
$$

where we note that differentiation of $\tilde{\mathcal{C}}$ along a horizontal curve $\gamma$ simply reads

$$
\frac{d \tilde{\mathcal{C}}}{d \xi_{t}}\left(\gamma\left(\xi_{t}\right)\right)=\theta^{\prime}\left(\xi_{t}\right) \tilde{\mathcal{C}}\left(\gamma\left(\xi_{t}\right)\right)+\left(\mathcal{A}_{2} \tilde{\mathcal{C}}\right)\left(\gamma\left(\xi_{t}\right)\right)
$$

Now if we divide by $\theta^{\prime}$ on both sides of (D.107) we can isolate $\theta^{\prime \prime}$ :

$$
\theta^{\prime \prime}=-\frac{\lambda_{3}}{\beta^{2} \tilde{\mathcal{C}}}\left(\left(\theta^{\prime}\right)^{2}+\beta^{2}\right)^{\frac{3}{2}}+\frac{\left(\tilde{\mathcal{C}}_{\theta}-\theta^{\prime} \mathcal{A}_{2} \tilde{\mathcal{C}}\right)\left(\left(\theta^{\prime}\right)^{2}+\beta^{2}\right)}{\beta^{2} \tilde{\mathcal{C}}} .
$$

Consequently, we may rewrite (D.105) in more explicit form:

$$
\left\{\begin{array}{l}
x^{\prime}=\cos (t \theta) \quad \text { for fixed } t \in[0,1], \beta>0, \\
y^{\prime}=t^{-1} \sin (t \theta) \\
\theta^{\prime}=v \\
v^{\prime}=-\frac{\lambda_{3}^{t}}{\tilde{C} \beta^{2}}\left(v^{2}+\beta^{2}\right)^{\frac{3}{2}}+\frac{\tilde{\mathcal{C}} \theta-v \mathcal{A}_{2} \tilde{\mathcal{C}}}{\tilde{\mathcal{C}} \beta^{2}}\left(v^{2}+\beta^{2}\right) .
\end{array}\right.
$$

Remark D.16. For $t \downarrow 0$ we get constant $\lambda_{3}^{t \downarrow 0}=\lambda_{3}^{0}=\beta c$. Now if we suppose $t=0$ and $\tilde{C}=1$ then the second term in the righthand-side of the last differential equation vanishes and we find

$$
\frac{\beta}{c} \int\left(v^{2}+\beta^{2}\right)^{-\frac{3}{2}} \mathrm{~d} v=\int 1 \mathrm{~d} x \Leftrightarrow \frac{1}{c \beta} \frac{v}{\sqrt{v^{2}+\beta^{2}}}=x+c_{2} \Leftrightarrow v(x)= \pm \frac{c_{1} \beta\left(x+c_{2}\right)}{\sqrt{\beta^{-4}-c_{1}^{2}\left(x+c_{2}\right)^{2}}},
$$

which explains the earlier formula (C.80).

\section{E Differences and analogies of (D.89) with Hamilton-Jakobi theory on integrable manifolds}

On an integrable manifold such as $\mathbb{R}^{n}$ one has the following canonical equations on phase space, [40],

$$
\begin{aligned}
\dot{q} & =\frac{\partial H}{\partial p} \\
-\dot{p} & =\frac{\partial H}{\partial q}
\end{aligned}
$$


where $H$ is the Hamiltonian of the system, corresponding to a non-homogenous Lagrangian system [40]ch:2. So we see that solving the canonical equations means following the flow of

$$
X_{H}=\left(\partial_{p} H,-\partial_{q} H\right) .
$$

Now set the following symplectic form on phase space $\omega_{0}=\mathrm{d} p \wedge \mathrm{d} q$ then

$$
\left.\left.X_{H}\right\rfloor \omega_{0}=X_{H}\right\rfloor \mathrm{d} p \wedge \mathrm{d} q=\partial_{p} H \mathrm{~d} q+\partial_{q} H \mathrm{~d} p=\mathrm{d} H .
$$

So the Hamiltonian flow preserves the symplectic volume form on phase space, since if we take the Liederivative of the symplectic form along the Hamiltonian flow we get

$$
\left.\left.\mathcal{L}_{X_{H}}=\mathrm{d} p \wedge \mathrm{d} q=X_{H}\right\rfloor \mathrm{~d}(\mathrm{~d} p \wedge \mathrm{d} q)+d\left(X_{H}\right\rfloor \mathrm{d} p \wedge \mathrm{d} q\right)=\mathrm{dd} H=0 .
$$

In section $\mathrm{D}$ we have a similar structure on the non-integrable contact manifold $Q$ rather than on the integrable manifold $\mathbb{R}^{n}$ in fact the 2 -form $\mathrm{d} \psi$ plays the role of the symplectic volume form $\omega_{0}$ on the phase space $Z=Q \times T\left((S E(2))_{t}\right)$ and the tangent-vectors $\gamma^{\prime}$ to the characteristic curves $\gamma$ relate to the Hamiltonian vector field $X_{H}$. The variables $\left\{\lambda_{i}, \lambda_{2}, \lambda_{3}\right\}$ relate to impuls variables and correspond to $q$, whereas the variables $\{x, y, \theta\}$ relate to position variable $p$.

For the optimization of homogenous Lagrangians $\mathcal{L}(p, \dot{p})=\sqrt{\sum_{i, j=1}^{n} g_{i j}(p, \dot{p}) \dot{p}^{i} \dot{p}^{j}}$, [40]ch:3, on integrable Riemannian manifolds of dimension $n<\infty$, the canonical equations take a slightly different form. In this case one has

$$
q_{i}=\mathcal{L} \frac{\partial \mathcal{L}}{\partial \dot{p}^{i}}, \quad i=1, \ldots, n
$$

with Lagrangian $\mathcal{L}^{2}(p, \dot{p})=\sum_{i, j=1}^{n} g_{i j}(p, \dot{p}) \dot{p}^{i} \dot{p}^{j}$. Now the corresponding Hamiltonian reads $H(p, q)=$ $\sum_{i, j=1}^{n} g^{i j}(p, q) q_{i} q_{j}$ where $g_{i j} g^{j k}=\delta_{i}^{k}$ and we have [40]p.147, eq. 1.29

$$
\begin{aligned}
& \dot{q}_{i}=-H \frac{\partial H}{\partial p^{i}}=-\frac{1}{2} \frac{\partial}{\partial p^{i}} H^{2} . \\
& \dot{p}^{i}=H \frac{\partial H}{\partial q_{i}}=-\frac{1}{2} \frac{\partial}{\partial q_{i}} H^{2},
\end{aligned}
$$

so for the homogenous case the canonical equations require $H^{2}$ rather than $H$.

Because of the non-integrability of the contact manifold $\left((S E(2))_{t}, \mathrm{~d} \mathcal{A}^{3}\right)$ and its extension $Q$, however, the canonical equations which hold on integrable manifolds no longer apply. However we do find somewhat similar equations to both $\dot{q}=\frac{\partial H}{\partial p}=\dot{q}$ and to $-\dot{p}=\frac{\partial H}{\partial q}$. These are respectively (D.96) and (D.95) (which are both parts of (D.89)), as we will motivate next.

The similar equations to the upper equations in (E.109) on our contact manifold $Q$, for the particular case $\tilde{C}=1$, are

$$
\left(\begin{array}{l}
\dot{x} \\
\dot{y}
\end{array}\right)=\left(\begin{array}{cc}
\cos \left(\alpha_{t} t\right) & -t \sin \left(\alpha_{t} t\right) \\
\frac{\sin \alpha_{t} t}{t} & \cos \left(\alpha_{t} t\right)
\end{array}\right)\left(\begin{array}{c}
\dot{\tilde{x}} \\
\dot{\tilde{y}}
\end{array}\right)=\left(\begin{array}{cc}
\cos \left(\alpha_{t} t\right) & -t \sin \left(\alpha_{t} t\right) \\
\frac{\sin \alpha_{t} t}{t} & \cos \left(\alpha_{t} t\right)
\end{array}\right)\left(\begin{array}{l}
\frac{\lambda_{3}^{t}}{\beta c} \\
\frac{\lambda_{2}^{t}}{\beta c}
\end{array}\right)
$$

where we recall (D.98), with $\cos \left(\alpha_{t} t\right)=-\frac{t \mu_{3}}{\beta c}, \sin \left(\alpha_{t} t\right)=\frac{\mu_{3}}{\beta c}$ which is determined by the boundary conditions of the characteristic curve.

Therefore we define

$$
\left(\begin{array}{c}
q_{2} \\
q_{3}
\end{array}\right)=\left(\begin{array}{c}
\lambda_{3}^{t} \cos \left(\alpha_{t} t\right)-t \lambda_{2}^{t} \sin \left(\alpha_{t} t\right) \\
\lambda_{2}^{t} \cos \left(\alpha_{t} t\right)-t^{-1} \lambda_{3}^{t} \sin \left(\alpha_{t} t\right)
\end{array}\right)
$$

and $H(q)=\sqrt{\frac{q_{2}^{2}+q_{3}^{2}}{\beta c}}$ and we indeed get

$$
\dot{x}=H \frac{\partial H}{\partial q_{2}} \text { and } \dot{y}=H \frac{\partial H}{\partial q_{3}} .
$$

However in the remaining direction we have $\dot{\theta}=\beta \frac{\lambda_{1}^{t}}{\lambda_{2}^{t}} \neq H \frac{\partial H}{\partial q_{2}}$, where we recall (D.91).

The similar equations to the last part of the canonical equations are given by (D.95). To this end we note that (D.95) may be rewritten as

$$
\dot{\lambda}_{1}^{t}=\frac{d \lambda_{1}^{t}}{d \xi_{t}}=-\lambda_{3}^{t}+\mathcal{A}_{1} \mathcal{L}, \quad \dot{\lambda}_{2}^{t}=\frac{d \lambda_{2}^{t}}{d \xi_{t}}=\frac{\beta^{2} \lambda_{3}^{t} \lambda_{1}^{t}}{\lambda_{2}^{t}}+\mathcal{A}_{2} \mathcal{L}, \quad \dot{\lambda}_{3}^{t}=\frac{d \lambda_{3}^{t}}{d \xi_{t}}=-\beta^{2} t^{2} \lambda_{1}^{t}+\mathcal{A}_{1} \mathcal{L} .
$$


where the Lagrangian $\mathcal{L}$ is given by $\mathcal{L}(\gamma, \dot{\gamma})=\sqrt{(\dot{\theta})^{2}+\beta^{2}} \tilde{C}(\gamma)$, where the first factor of the Lagrangian depends only on $\dot{\gamma}$ and where the second factor of the Lagrangian depends only on $\gamma$. Now in the standard Hamilton Jakobi theory on integrable manifolds this would imply that the Hamiltonian is given by $H(g, \lambda)=\beta \tilde{\mathcal{C}}(g) \sqrt{\left(\frac{\lambda_{1}^{t}}{\lambda_{2}^{t}}\right)^{2} \beta^{2}+1}$, so that

$$
\dot{\lambda}_{1}^{t}=\mathcal{A}_{1} H-\lambda_{3}^{t}, \quad \dot{\lambda}_{2}^{t}=\mathcal{A}_{2} H+\frac{\beta^{2} \lambda_{3}^{t} \lambda_{1}^{t}}{\lambda_{2}^{t}}, \quad \dot{\lambda}_{3}^{t}=\mathcal{A}_{3} H-\beta^{2} t^{2} \lambda_{1}^{t} .
$$

On the one hand the Pfaffian system (D.89) on our contact manifold $Q$ takes the same role as the canonical equations which hold along characteristic curves (E.109) of an Euler-Lagrange system on an integrable manifold, whereas on the other hand (E.110) and (E.111) reveal substantial differences of (D.89) in comparison to (E.109), due to the non-integrability of the contact manifold $\left((S E(2))_{t}, \mathrm{~d} \mathcal{A}_{t}^{3}\right)$ (and its extension $Q$ ).

\section{F $\quad \mathcal{G}_{\beta}$-Orthogonality of the horizontal, left-invariant diffu- sions in Section 7 and horizontal, left-invariant convec- tion in Section 5 by Cauchy-Riemann relations.}

As noted in the introduction and section 2 the domain of a Gabor transform should neither be considered as $H_{3}$ nor as $H_{3} / \mathbb{T}$ but rather as the principal fiber bundle $P_{\mathbb{T}}=\left(H_{3}, \mathbb{T}, \pi, R\right)$. Alternatively, if one is only interested on the structure on the tangent space, one can consider the contact manifold $\left(H_{3}, \mathrm{~d} \mathcal{A}^{3}\right)$, recall Definition B.14. To this end we recall that the reason for these considerations on the domain of a Gabor transform is that in both the left-invariant diffusions on the Gabor transforms (explained in Section 7) and the left-invariant convection on the Gbaor transforms (explained in Section 6) take place along horizontal curves, recall Definition B.12, only. This means that we omit $\mathcal{A}_{3}$ as a possible direction in each tangent space. This omission does not correspond to considering the coset space $H_{3} / \mathbb{T} \sim \mathbb{R}^{2}$. To this end we note that horizontal curves have a specific non-trivial third $s$-component (in contrast to their tangent vectors). Moreover, the vector fields $\mathcal{A}_{1}, \mathcal{A}_{2}$ do not commute and the third $s$-axis should intuitively be considered as a buffer which stores the non-commutative nature of moving in $\mathcal{A}_{1}$ and $\mathcal{A}_{2}$-direction, i.e. it stores the uncertainty between position and frequency in the Gabor domain, recall (1.3).

On $P_{\mathbb{T}}$ we imposed the first fundamental form $G_{\beta}$, recall (C.74). By means of this first fundamental form we can express the Cauchy-Riemann relations (2.32) that hold between local phase and local amplitude are for $\beta^{-1}=a$ simply as

$$
\mathcal{G}_{\beta=\frac{1}{a}}^{-1}(\mathrm{~d} \log |U|, \mathrm{d} \Omega)=0,
$$

where the left-invariant gradient equals $\mathrm{d} \Omega=\sum_{i=1}^{3} \mathcal{A}_{i} \Omega \mathrm{d} \mathcal{A}^{i}$ and where $U=\mathcal{W}_{\psi_{a}} f$ with $\psi_{a}(\xi)=e^{-a^{-2} \xi^{2} n \pi}, a>$ 0 . which gives us a geometric understanding of this fundamental form. The normal vector $\left.\mathrm{d} \Omega\right|_{g_{0}}$ to the surface $\left\{(p, q, s) \in H_{r} \mid \Omega(p, q, s)=\right.$ constant $\left.=\Omega\left(g_{0}\right)\right\}$ is $\mathcal{G}_{\beta}$-orthogonal to the normal vector $\mathrm{d} \mid U \|_{g_{0}}$ to the surfaces $\left\{(p, q, s) \in H_{r}|| U \mid(p, q, s)=\right.$ constant $\left.=|U|\left(g_{0}\right)\right\}$.

Now let us consider the consequences of this observation in our convection and diffusion schemes, which only use the horizontal part of each tangent space. Clearly convection takes place in the horizontal direction $\mathcal{G}_{\beta}$-orthogonal to phase-gradient $\mathrm{d} \Omega$, so that the convection on the Gabor transform takes place along iso-contours of the phase, so that phase covariance coincides with phase invariance. On the other hand the principal horizontal direction of the diffusion equals the eigen vector with smallest eigen value of the structure tensor of the absolute value, which (for $\sigma \downarrow 0$ ) is $\mathcal{G}_{\beta}$-orthogonal to the left-invariant gradient of the amplitude, since $\left(\mathcal{A}_{1}|U|, \mathcal{A}_{2}|U|\right)=\left(\partial_{p}|U|, \partial_{q}|U|\right)$. Consequently, by (F.112) the principal anisotropy direction of the diffusion is $\beta$-orthogonal to the direction of convection (adaptive transport). See Figure 14. 


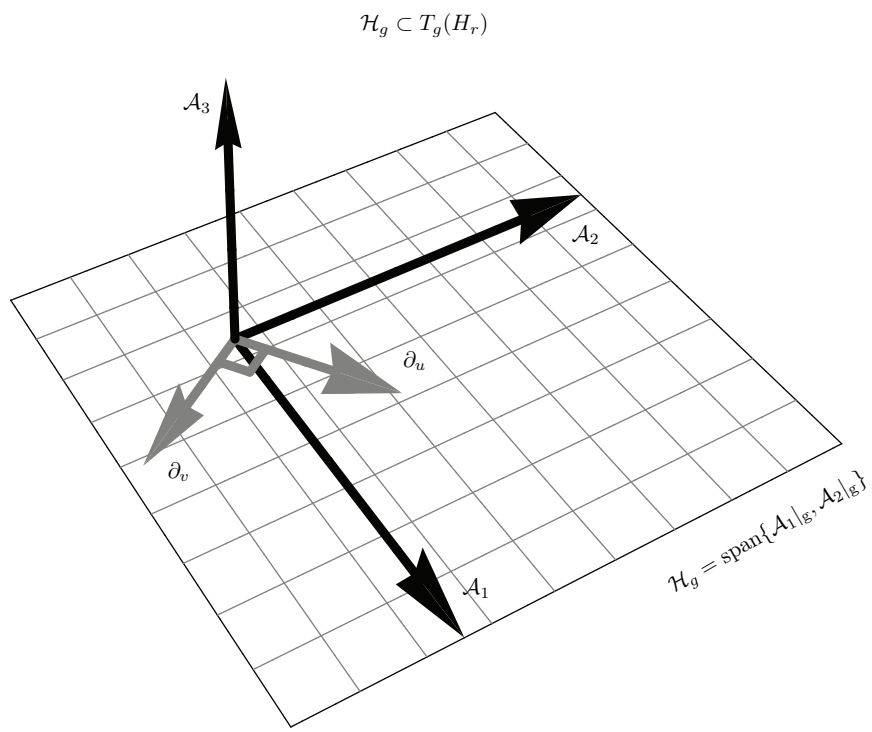

Figure 14: The horizontal direction $\partial_{u}$ in each tangent space $T_{g}\left(H_{r}\right), g \in H_{r}$ along which the convection described in Section 5 takes place is $\beta$-orthogonal to the horizontal principal direction $\partial_{v}$ of anisotropic left-invariant diffusion described in section 7. Note that $\operatorname{span}\left\{\partial_{u}, \partial_{v}\right\}=$ $\operatorname{span}\left\{\left.\mathcal{A}_{1}\right|_{g},\left.\mathcal{A}_{2}\right|_{g}\right\}=\operatorname{ker}\left(\omega_{g}\right)=: \mathcal{H}_{g}$, where $\mathcal{H}_{g} \subset T_{g}\left(H_{r}\right)$ denotes the horizontal part of the tangent space $T_{g}\left(H_{r}\right)$, which also coincides with the tangent space to the principal fiber bundle $P_{\mathcal{T}}$ and which is also the kernel of the Cartan-Ehresmann connection $\omega_{g}$ at $g \in H_{r}$ given by (B.66). Now from (F.112) it now follows the horizontal local Gauge-fields $\left\{\partial_{u}, \partial_{v}\right\}$ from Section 7 are (at least for $\sigma \downarrow 0$ ) respectively aligned with horizontal equi-phase and equi-amplitude lines in $H_{r}$.

\section{G Explanation on formula (2.11)}

The somewhat uncommon explicit formula of the Lie product on the tangent space $T_{e}(G)$ of the unity element $e$ of a matrix group $G$, formula (2.11), follows directly from the CBH-formula:

$$
\begin{aligned}
h^{-2}\left(e^{h A} e^{h B} e^{-h A} e^{-h B}-I\right) & =h^{-2} e^{h A}\left(e^{h B} e^{-h A} e^{-h B}-e^{-h A}\right) \\
& =h^{-2}\left(e^{h^{2}[A, B]+O\left(h^{3}\right)}-I\right) \\
& =h^{-2}\left(I+h^{2}[A, B]-I+O\left(h^{3}\right)\right) \\
& =[A, B]+O(h),
\end{aligned}
$$

for all $A, B \in T_{e}(G)$.

\section{References}

[1] M. Akian and J. Quadrat and M. Viot. Bellman Processes. Lecture Notes in Control and Information Science, Springer-Verlag, 199: 302-311, 1994.

[2] F. Auger and P. Flandrin. Improving the readability of time-frequency and time-scalerepresentations by the reassignment method. IEEE Transactions on Signal Processing, 43(5): 1068-1089, May 1995.

[3] R. Bryant and P. Griffiths. Reduction for constrained variational problems and $(1 / 2) \int \kappa^{2}$ ds. American Journal of Mathematics, 108(3):525-570, June 1986.

[4] R. Bryant and P. Griffiths and D. Grossman. Exterior Differential Systems and Euler-Lagrange Partial Differential Equations. Chicago lectures in mathematics, Chicago, USA, 2003.

[5] B. Burgeth and J. Weickert An Explanation for the Logarithmic Connection between Linear and Morphological Systems. Lecture Notes in Computer Science, SpringerVerlag, Proc. 4th int. Conference Scale Space, p. 325-339, 2003.

[6] M. A. Akivis and B.A. Rosenfeld. Élie Cartan (1869-1951), volume 123. American Mathematical Society, Providence, 1993. Translations of Mathematical Monographs. 
[7] E. Chassande-Mottin, I. Daubechies, F.Augur and P.Flandrin Differntial reassignment. IEEE Signal proc. Lett. 4, pp.293-294, 1997.

[8] I. Daubechies and S. Maes. A nonlinear squeezing of the continuous wavelet transform based on auditory nerve models. In Wavelets in Medicine and Biology, pages 527-546, CRC Press, 1996.

[9] L. Daudet, M. Movidone, and B. Torrésani. Time-Frequency and Time-Scale Vector Fields for Deforming Time-Frequency and Time-Scale representations citeseer.ist.psu.edu/372594.html

[10] J. Dieudonné. Treatise on Analysis AP, New York, 1977, Vol. V.

[11] R. Duits. and E.M. Franken Left invariant parabolic evolution equations on SE(2) and contour enhancement via invertible orientation scores, part I: Linear left-invariant diffusion equations on $S E(2)$ Submitted to the Quarterly on Applied mathematics, AMS, 2008.

[12] R. Duits. and E.M. Franken Left invariant parabolic evolution equations on SE(2) and contour enhancement via invertible orientation scores, part II: Nonlinear left-invariant diffusion equations on invertible orientation scores Submitted to the Quarterly on Applied mathematics, AMS, 2008.

[13] R. Duits. Perceptual Organization in Image Analysis. PhD thesis, Eindhoven University of Technology, Department of Biomedical Engineering, The Netherlands, 2005. A digital version is available on the web URL: http:// www.bmi2.bmt.tue.nl/Image-Analysis/People/RDuits/THESISRDUITS.pdf.

[14] R.Duits, B.Burgeth. Scale Spaces on Lie groups Proceedings, of SSVM 2007, 1st international conference on scale space and variational methods in computer vision, Lecture Notes on Computer Science, p.300-312, editors: Sgallari, Murli and Paragios, Springer-Verlag, June 2007

[15] R. Duits and M. van Almsick. The Explicit Solutions of Linear Left-Invariant Second Order Stochastic Evolution Equations on the 2D-Euclidean Motion Group Quarterly of Applied Mathematics, Vol. 66, p.27-67, 2008.

[16] R. Duits, M. Felsberg, G. Granlund, and B.M. ter Haar Romeny. Image analysis and reconstruction using a wavelet transform constructed from a reducible representation of the euclidean motion group. International Journal of Computer Vision. Volume 72, issue 1, p.79-102, April 2007.

[17] R.Duits and E.M. Franken Left-invariant Stochastic Evolution Equations on $S E(2)$ and its Applications to Contour Enhancement and Contour Completion via Invertible Orientation Scores. arXiv: 0711.0951v4, see http://arxiv.org/abs/0711.0951 Also available as CASA report nr.35, 2007, Eindhoven University of Technology, http://www.win.tue.nl/casa/research/casareports/2007.html

[18] E.M. Franken and R. Duits Crossing-Preserving Coherence-Enhancing Diffusion on Invertible Orientation Scores. Accepted for publication in International Journal of Computer Vision, to apear 2009.

[19] E.M. Franken. Enhancement of Crossing Elongated Structures in Images. PhD-thesis, Dep. of Biomedical Engineering, Eindhoven University of Technology, The Netherlands, October 2008

[20] H. Führ. Abstract Harmonic Analysis of Continuous Wavelet Transforms Springer Heidelberg-New York, 2005.

[21] D. Gabor. Theory of communication. Journal of the Institution of Electrical Engineers, 93(22):429457, November 1946.

[22] B. Gaveau. Principe de moindre action, propagation de la chaleur et estimees sous elliptiques sur certains groupes nilpotents. Acta mathematica, 139:96-153, 1977.

[23] K. Gröchenig. Foundations of Time-Frequency Analysis. Birkhauser, first edition, 2001.

[24] A. Grossmann, J. Morlet, and T. Paul. Integral transforms associated to square integrable representations. J.Math.Phys., 26:2473-2479, 1985.

[25] W. Hebisch. Estimates on the semigroups generated by left-invariant operators on lie groups. Journal fuer die reine und angewandte Mathematik, 423:1-45, 1992.

[26] C.W. Helström. An expansion of a signal in gaussian elementary signals. IEEE Transactions on Information Theory, 12:81-82, January 1966.

[27] H. Bölcskei and F. Hlawatsch. Discrete Zak Transforms, Polyphase Transforms, and Applications. IEEE Transactions on signal processing, 45:4:851-866, 1997.

[28] L. Hormander. Hypoellptic second order differential equations. Acta Mathematica, 119:147-171, 1968.

[29] A.J.E.M. Janssen. The Zak transform: A signal transform for sampled time-continuous signals. Philips J. Res., vol. 43, p.23-69, 1988. 
[30] A.J.E.M. Janssen. Duality and biorthogonality for Weyl-Heisenberg frames. Journal of Fourier Analysis and applications, vol.1, no.4, p.403-437, 1995.

[31] J. Jost. Riemannian Geometry and Geometric Analysis 4th edition, Springer Heidelberg-New York, 2005.

[32] M.Kass and A.Witkin and D.Terzopoulos", Snakes: Active Contour Models, IJCV, Vol. 1, Nr. 4, 321-331, 1988.

[33] K. Kodera, C. de Villedary, and R. Gendrin. A new method for the numerical analysis of nonstationary signals. Physics of the Earth and Planetary Interiors, 12:142-150, August 1976.

[34] G.W. Mackey, Imprimitity for representations of locally compact groups Proc. N.A.S. 35: p.537-545, 1949.

[35] J. Marsden and A. Weinstein. Reduction of symplectic manifolds with symmetry. Rep. Math. Phys., 5:121-130, 1974.

[36] D. Mumford. Elastica and computer vision. Algebraic Geometry and Its Applications. Springer-Verlag, pages 491-506, 1994.

[37] P. Perona and J. Malik. Scale-space and edge detection using anisotropic diffusion. IEEE-transactions on Pattern Analysis and Machine Intelligence, (7).

[38] F. Monroy-Perez and J. Anzaldo-Meneses. Optimal control on the Heisnebreg group. Journal of dynamical and control systems, 5(4), 1999, 473-499.

[39] H.G.J. Pijls. The Yang-Mills equations. In proc. seminar 1981-1982 mathematical structures in field theories, ed. E.M. de Jager and H.G.J. Pijls, p.119-182, 1984

[40] H. Rund. The Hamilton-Jacobi theory in the calculus of variations. D. van Nostrand, London, 1966.

[41] M. Spivak. Differntial geometry Vol II, Publish or Perish inc. 1975.

[42] A.F.M. ter Elst and D.W. Robinson Weighted Subcoercive Operators on Lie Groups Journal of Functional Analysis, 157:88-163, 1998.

[43] M.E. Testorf, J. Ojeda-Castaneda, and A.W. Lohmann, editors. Selected Papers on Phase-Space Optics, volume MS181 of SPIE Milestone Series. The Society of Photo-Optical Instrumentation Engieers, Bellingham, Washington USA, 2006

[44] V.S. Varadarajan. Lie Groups, Lie-Algebras and Their Representations. Springer-Verlag, New York, Berlin, Heidelberg, Tokyo, 1984.

[45] J. A. Weickert. Anisotropic Diffusion in Image Processing. ECMI Series. Teubner, Stuttgart, January 1998.

[46] J. A. Weickert. Coherence-enhancing diffusion filtering. International Journal of Computer Vision, 31(2/3):111-127, April 1999.

[47] S.T. Ali and J.P. Antoine and J.P. Gazeau Coherent States, Wavelets and Their Generalizations Springer Verlag, New York, Berlin, Heidelberg, 1999. 